\title{
Design and synthesis of Coenzyme $A$ analogues as Aurora kinase $A$ inhibitors: an exploration of the roles of the pyrophosphate and pantetheine moieties
}

Fiona Bellany, ${ }^{\mathrm{a}, \mathrm{b}}$ Yugo Tsuchiya, ${ }^{\mathrm{c}}$ Trang M. Tran, ${ }^{\mathrm{a}}$ A. W. Edith Chan, ${ }^{\mathrm{d}}$ Helen Allan, ${ }^{\mathrm{a}}$ Ivan Gout ${ }^{\mathrm{c}}$ and Alethea B. Tabor ${ }^{\text {** }}$

aDepartment of Chemistry, UCL, Christopher Ingold Building, 20, Gordon Street, London WC1H OAJ, UK

bPresent Address: Drug Discovery Unit, Division of Biological Chemistry and Drug Discovery, University of Dundee, Dundee, DD1 5EH, UK

'Department of Structural and Molecular Biology, UCL, Gower Street, London WC1E 6BT, UK

${ }^{d}$ Wolfson Institute for Biomedical Research, University College London, London WC1E 6BT, UK

\section{Introduction}

The Aurora kinases are a family of highly homologous serine/threonine kinases, ${ }^{1,2,3}$ which have welldocumented roles in the control of meiosis, mitosis and cell division. ${ }^{4}$ There are three distinct subfamilies, Aurora A, Aurora B and Aurora C, which vary in their function and subcellular location. Since the first observation of the overexpression of Aurora kinases in cancer cell lines by Bischoff et al., ${ }^{5}$ they have been regarded as promising drug targets for cancer chemotherapy. Aurora A overexpression is frequently detected in leukemia, breast, prostate and colon cancers, ${ }^{2,5,6}$ with a lower overall survival rate seen in colorectal cancer patients with increased Aurora A levels. ${ }^{7}$ This has seen a number of Aurora kinase inhibitors progressing through different stages of clinical trials., ${ }^{3,8,9,10,11}$ The Aurora kinases are highly homologous, with a highly conserved $C$-terminal catalytic domain, a short $N$-terminal domain which varies in size, and an activation loop (Asp274-Glu299) which is conserved between the three family members. ${ }^{3}$ The kinase activity of Aurora $A$ is dependent on autophosphorylation of Thr288 (and possibly Thr287), found in the activation loop. In addition, binding of Aurora A to microtubule-associated proteins, in particular TPX2, alters the structure of Aurora A by stabilisation of the activation loop, allowing ATP to bind and driving the kinase into the optimal conformation for catalysis. ${ }^{1,3,12}$ Inhibition of Aurora A results in inhibition of this phosphorylation, giving delayed entry into mitosis and the failure of the centrosomes to assemble bipolar spindles, resulting in aneuploidy and mitotic arrest. ${ }^{13}$ Different conformational states of Aurora A can be induced or stabilised by different small molecule inhibitors, ${ }^{14,15,16,17}$ and in particular the conformation of the activation loop modulates the interaction of Aurora A with its binding partners. ${ }^{18}$ 
Coenzyme A ( $C O A)$ is an essential and ubiquitous cofactor made from vitamin B5 (pantothenate), ATP, and cysteine. CoA and its thioester derivatives (Acetyl CoA, Malonyl CoA, HMG CoA among others) are involved in diverse anabolic and catabolic pathways, biosynthesis of neurotransmitters and the regulation of gene expression. ${ }^{19,20}$ Dysregulation of CoA biosynthesis or CoA thioester homoeostasis is associated with various human pathologies, including neurodegeneration, cancer and metabolic disorders. ${ }^{21-24}$ Recent studies have uncovered a novel function of CoA in redox regulation, involving covalent modification of cellular proteins by disulphide bond formation, termed CoAlation, in cellular response to oxidative and metabolic stress. ${ }^{25,26}$ Protein CoAlation is a widespread and reversible posttranslational modification, which occurs in single-cell and multicellular organisms, and modulates catalytic activity, regulatory interactions, subcellular localization and the stability of modified proteins. $^{27-30}$

Recently, we have reported that CoA is a specific ATP-competitive Aurora A inhibitor in vitro. ${ }^{31}$ Using a combination of biochemical, cell biology and biophysical studies, combined with the determination of the X-ray crystal structure of CoA with Aurora A (PDB: 6I2U) we demonstrated that the ADP moiety of CoA occupies the canonical ATP binding site of Aurora A, while the extended CoA pantetheine tail stretches away from the ATP site towards the kinase activation loop, forming a disulfide bond with the side chain of Cys290 adjacent to the Thr288 phosphorylated residue (Figure 1b). The pantothenic acid moiety of CoA interacts with the tip of the Gly-rich loop (residues 142-145) and displaces it from the position found in Aurora A/ADP complexes. The 3'-phosphate group of CoA is also ideally positioned to form a H-bond with the side chain of Thr217. Both the phosphate ADP moiety and the thiol group of $\mathrm{COA}$ are involved in mediating specificity determining interactions with Aurora $\mathrm{A}$ via targeting of Thr217 and Cys290 respectively. This dual-mode action of Aurora A inhibition by CoA traps the kinase in an inhibitory conformation, thus making CoA an irreversible inhibitor of Aurora A. 

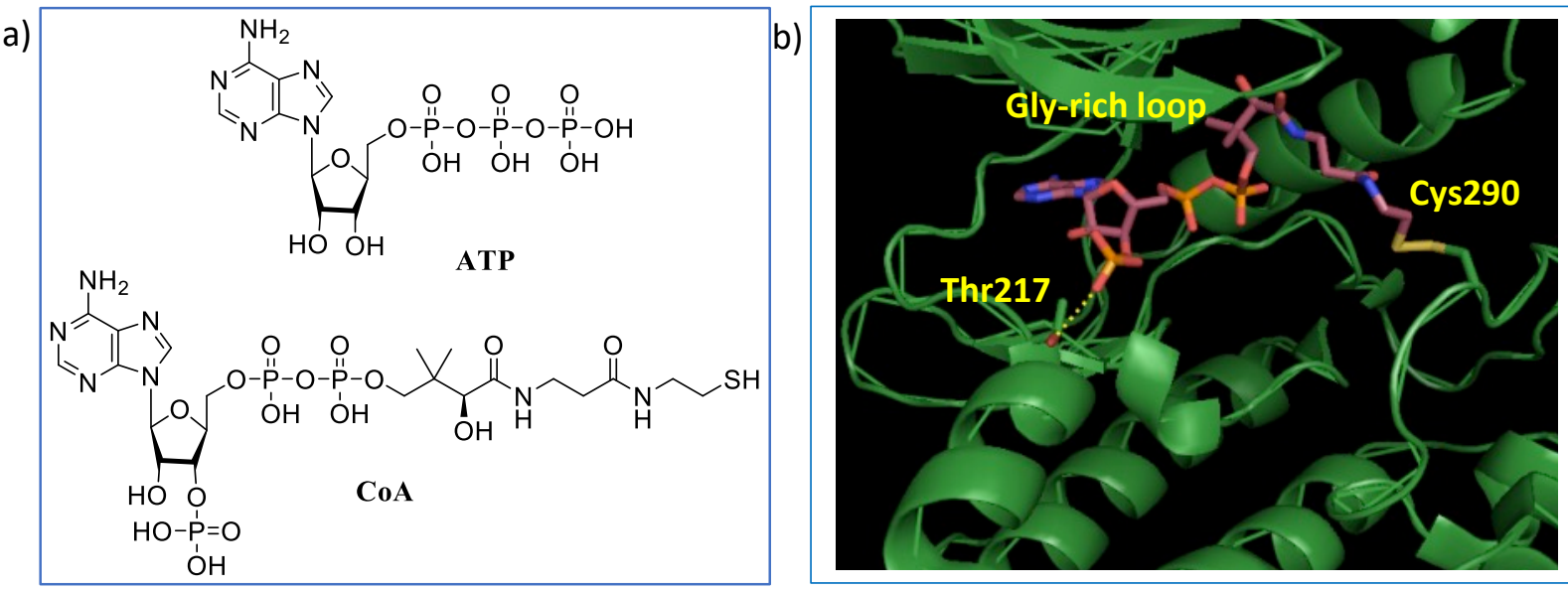

c)

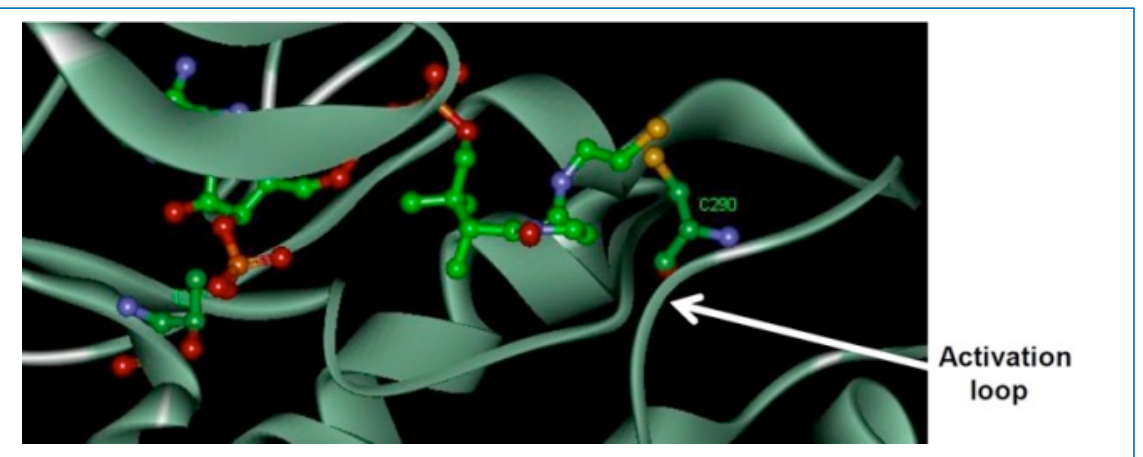

Figure 1: (a) Structural similarities of ATP and CoA; (b) Crystal structure of Aurora A in complex with CoA highlighting the Thr217 and Cys290 side chains (PDB: 6I2U); (c) Model of CoA bound to Aurora A (PDB 1OL7). The location of Thr217 adjacent to the 3'phospho-adenine of CoA, and Cys290 in the kinase activation segment, are both indicated.

This unique interaction between CoA and Aurora A kinase presents an opportunity to design novel selective inhibitors of Aurora A kinase. In this paper, we have undertaken the design and synthesis of potential selective inhibitors of Aurora kinase A, based on the CoA structure, exploring in particular the structure-activity relationships of the pyrophosphate and pantetheine moieties of CoA.

\section{Results and discussion}

\subsection{Design of pyrophosphate isostere analogues of CoA}

Based on the structural similarities between ATP and CoA (Figure 1a), and detailed biochemical investigations of the interactions between CoA and Aurora kinase $A,{ }^{31}$ we were also able to predict the binding mode of CoA into Aurora A by molecular docking studies using GOLD (Genetic Optimisation for Ligand Docking). ${ }^{32,33}$ A PDB file (1OL7) that corresponds to the published crystal structure data of 
the active, phosphorylated form of human Aurora A kinase ${ }^{12}$ was modified using $\mathrm{MOE}^{34}$ to include the cysteine residue Cys290, present in the activation loop of Aurora A (Figure 1c). The results of the docking were in good agreement with the X-ray crystal structure of the complex. In particular, the $3^{\prime}$ phosphate group of CoA is able to interact with the side chain of Thr 217 , and in the majority of the docking poses the pantetheine tail extends towards a front pocket of the active site in proximity to the side chain of Cys290. We were thus able to use this modified crystal structure as a basis for designing potential selective inhibitors or Aurora A kinase, based on modified CoA structures.

CoA has several limitations as a lead structure for drug design, ${ }^{35,36}$ in particular its large size, the presence of charged phosphate groups which would prevent it from crossing the cell membrane, and the possibility of off-target effects due to its use in many metabolic pathways. Initially therefore we elected to explore structures with the 3'-phosphate group removed and incorporating mimics of the pyrophosphate group, in order to make a more drug-like CoA structure. Several reviews of phosphate and pyrophosphate mimics have been published. ${ }^{37,38,39}$ Phosphate isosteres include phosphonates, sulfamoyl groups, carboxylate groups and heterocycles such as triazoles and thiazolidinones. Despite the literature, there is no clear "gold-standard" pyrophosphate mimic. Isosteres based on the carboxylate group have improved cell permeability when compared to the phosphate group, ${ }^{39}$ and are frequently more synthetically accessible. In this work, we focused our attention on dicarbonyl mimics of the pyrophosphate group ${ }^{40,41}$ combining these with a conserved adenosine headgroup and a variety of pantetheine tail groups (Table 1). To assess the drug-likeness of these structures, as CoA and many of these analogues contain ionizable groups, we calculated the $\log D$ values using Stardrop ${ }^{42}$ and compared these, the MW, HBD and HBA with CoA. This showed a significant improvement in lipophilicity for 3, $\mathbf{5}$ and $\mathbf{1 0}$ compared with CoA. However, the logD values of these compounds still lie below the desirable range of $1<\log D<3$ in which cell permeability and compound solubility are optimized..$^{43}$

Table 1. Aurora A inhibitor analogues of CoA.

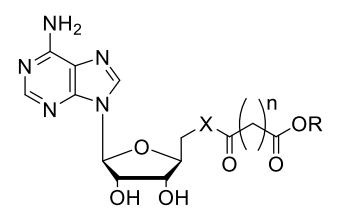




\begin{tabular}{|c|c|c|c|c|c|c|c|}
\hline Compound & $x$ & $\mathrm{n}$ & $\mathrm{R}$ & $\log \mathrm{D}$ & MW & $\mathrm{HBD}^{\mathrm{a}}$ & $\mathrm{HBA}^{\mathrm{b}}$ \\
\hline 1 & 0 & 1 & & 0.39 & 595.6 & 6 & 17 \\
\hline 2 & $\mathrm{O}$ & 1 & $\mathrm{H}$ & -2.34 & 353.3 & 4 & 12 \\
\hline 3 & $\mathrm{~N}$ & 1 & & 0.59 & 594.6 & 7 & 17 \\
\hline 4 & $\mathrm{~N}$ & 1 & $\mathrm{H}$ & -2.39 & 352.3 & 5 & 12 \\
\hline 5 & 0 & 2 & & 0.54 & 609.6 & 6 & 17 \\
\hline 6 & 0 & 3 & & 0.70 & 623.7 & 6 & 17 \\
\hline 7 & 0 & 2 & x & 0.35 & 437.4 & 3 & 12 \\
\hline 8 & 0 & 2 & & -0.28 & 510.5 & 5 & 15 \\
\hline 9 & 0 & 2 & & 0.18 & 581.6 & 6 & 17 \\
\hline 10 & 0 & 2 & & 0.57 & 627.7 & 6 & 17 \\
\hline COA & - & - & - & -0.05 & 767.5 & 9 & 23 \\
\hline
\end{tabular}

aHBA: No. of H Bond Acceptors, ${ }^{\mathrm{b}} \mathrm{HBD}$ : No. of H bond donors.

The suitability of analogues bearing a dicarbonyl linker was initially evaluated by docking these analogues into the Cys290-modified, active, phosphorylated form of human Aurora A kinase described above. Initial docking studies using analogue 1 suggest that the adenosine region binds to the ADPbinding pocket as expected. However, 1 does not have a particular preference for the position of the tail region, either extending towards the back pocket of Aurora A or potentially interacting with Cys 290 (Figure 2a,b), possibly because the analogue lacks the $-\mathrm{SH}$ warhead. Figure $2 \mathrm{c}$ shows the highest CHEMPLP GOLD score result, the required hydrogen bonding between the Ala213 in the hinge region of the kinase and the adenine of $\mathbf{1}$ can be clearly seen. There is also a potential interaction between one of the carbonyl groups in the linker and either Glu260/Asn261. It is also apparent that the $3^{\prime} \mathrm{OH}$ is 
too far away to pick up an interaction with Thr217 to improve selectivity towards Aurora A. Although the result in Figure 2 is shown for the binding mode where the tail extends into the back pocket, these interactions were consistent throughout all the results.

In addition to analogue $\mathbf{1}$ we aimed to investigate the SAR of varying the pyrophosphate and pantetheine moieties by synthesising analogues with substitution of an ester bond with an amide (3), different dicarbonyl linker lengths $(5,6)$ and tail lengths $(2,4,7,8,9)$. It is possible that with the addition of a suitable group to the terminal region of the tail, the preferred binding mode with interaction with Cys 290 could be achieved. This interaction is believed to be an important requirement for potency and selectivity towards Aurora A and this could be exploited in this structure by switching the terminal $\mathrm{CH}_{3}$ group to an $\mathrm{SH}$ group $(\mathbf{1 0}$, as in $\mathrm{CoA})$. It is also worth noting that the addition of an extra moiety to the $3^{\prime} \mathrm{OH}$ is required to pick up an interaction with Thr217 to improve the selectivity towards Aurora A. However, in the first instance, it was envisaged that compounds would be synthesised without the addition of these groups to identify simplified lead compounds for the inhibition of Aurora A.
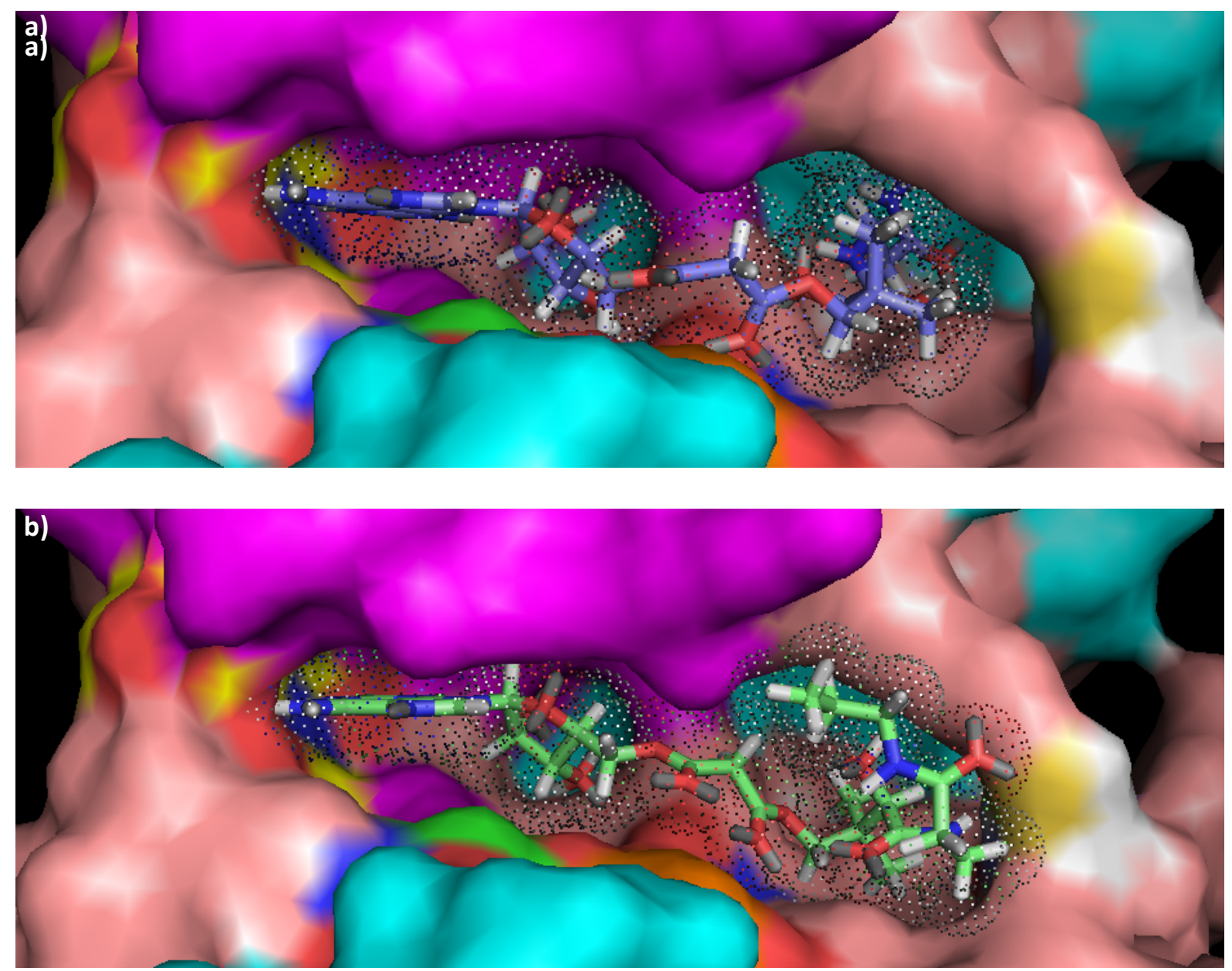


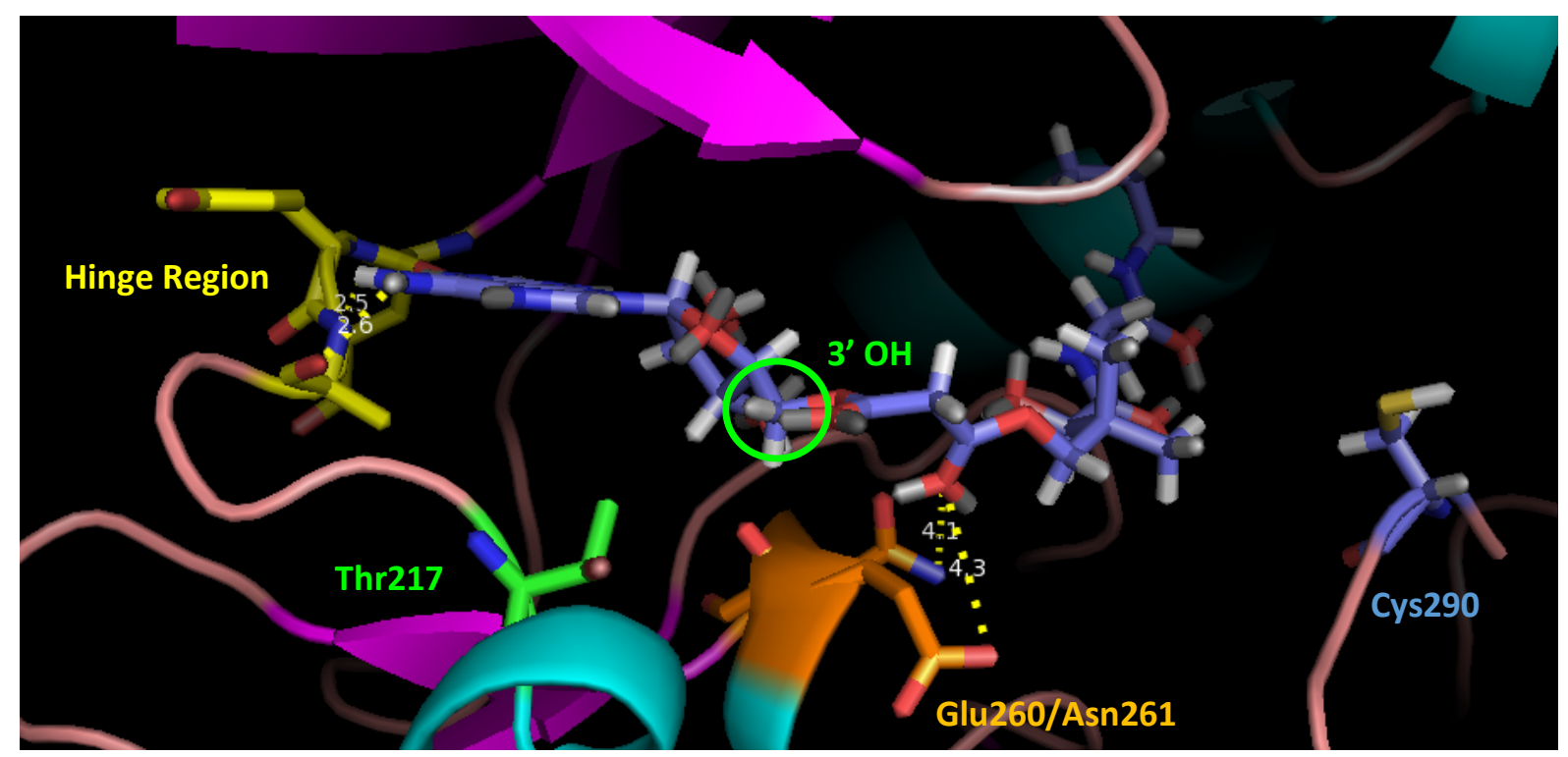

Figure 2: Docking results for the binding of 1 to Aurora A, showing the two possible tail binding modes: a) binding of the tail into the back pocket of the kinase and b) the binding of the tail to the solvent front of the kinase where it is possible to pick up an interaction with Cys290, c) This figure highlights the potential hydrogen bonding between the adenine of 1 and the hinge region of the kinase and the interactions between the linker moiety and Glu260/Asn261.

\subsection{Synthesis of pyrophosphate isostere analogues of Coenzyme $A$}

Initially the synthesis of the dicarbonyl analogue 1 was attempted using adenosine protected at the 2'/3' positions with an acetonide protecting group, however, it was found that removal of this protecting group in the final step was not possible due to the degradation of the pantetheine tail under a range of acidic conditions. A range of different protecting groups were tried, and the desired dicarbonyl analogue 1 was finally synthesised using adenosine protected with the tetraisopropyldisilyl (TIPDS) group (Scheme 1). 


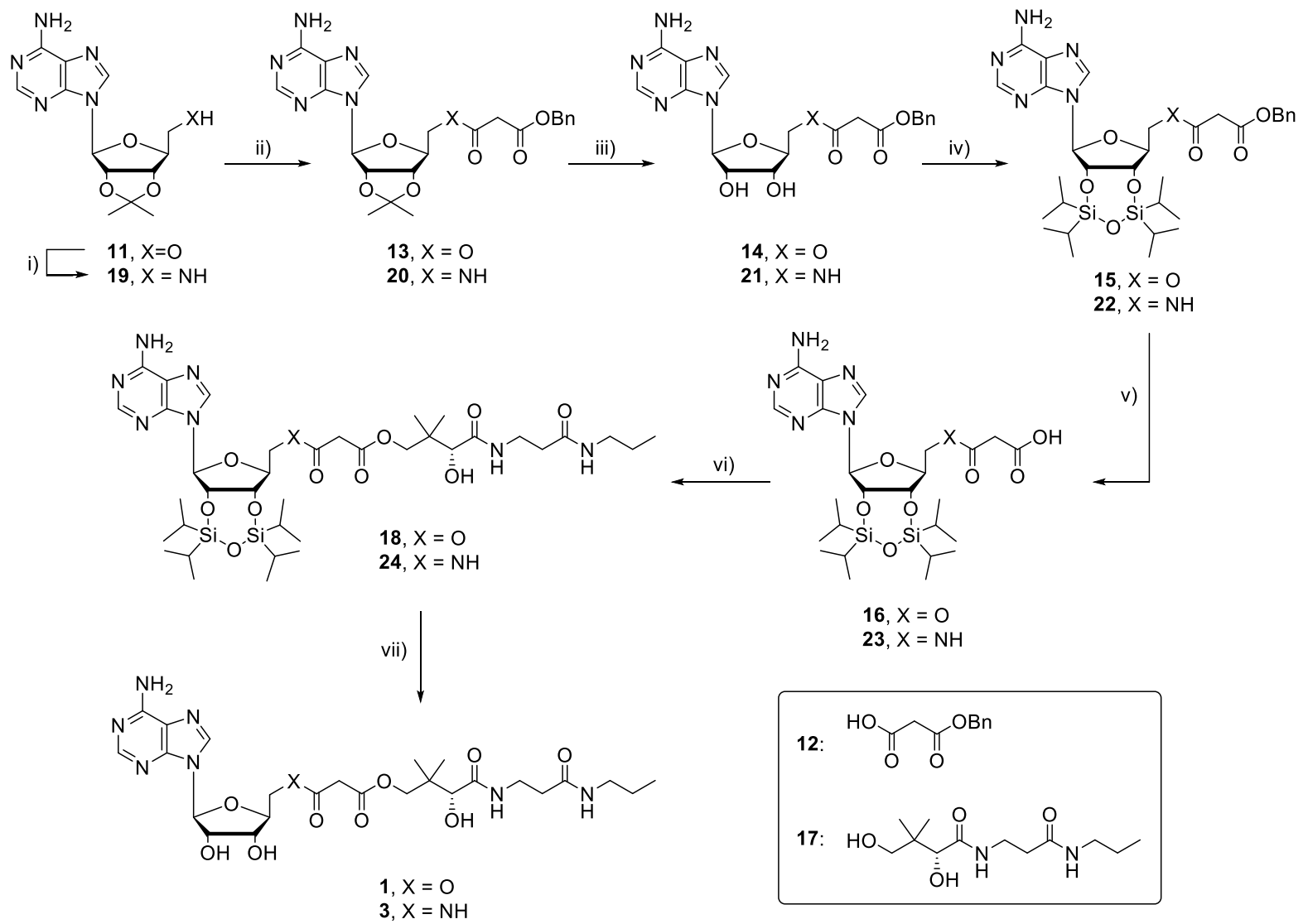

Scheme 1: Synthesis of $\mathbf{1}$ and $\mathbf{3}$ using the TIPDS protecting group. Conditions: (i) 1.1 eq. phthalimide, 1.1 eq. $\mathrm{PPh}_{3}, 1.1$ eq. DIAD, THF, RT, $5 \mathrm{~h}$ then 5.0 eq. hydrazine hydrate, EtOH, reflux, $16 \mathrm{~h}, 44 \%$ over two steps; (ii) 2.0 eq. 12, 25 mol\% DMAP, 1.3 eq. DCC, $\mathrm{CH}_{2} \mathrm{Cl}_{2}$, RT, 19 h, 13: 52\%, 20: 78\%; (iii) TFA, $\mathrm{H}_{2} \mathrm{O}, \mathrm{RT}, 3-4$ h, 14: 80\%, 21 : 90\%; (iv) 15: 1.1 eq. TIPDSCl$_{2}$, pyridine, RT, 18 h, 45\%; 22: 1.2 eq. TIPDSCl $2,2.5$ eq. imidazole, 25 mol\% DMAP, DMF, RT, 17 h, 61\%; (v) 10 mol\% Pd/C, MeOH, RT,3-5 h, 16: 71\%, 23: 95\%; (vi) 1.0 eq. 17, 1.2 eq. EDC, 1.2 eq. DMAP, RT, 18-20 h, 18: 25\%, 24: 14\%; (vii) 8.5 eq. $\mathrm{NH}_{4} \mathrm{~F}, \mathrm{MeOH}, \mathrm{RT}, 1.5$ h, 1: 42\%, 2: $75 \%$.

Linker 12 was prepared in 71\% yield via ring-opening of Meldrum's acid with benzyl alcohol. ${ }^{44}$ Esterification ${ }^{45,46}$ of 2',3'-isopropylidene adenosine (11) with protected linker $\mathbf{1 2}$ was followed by the removal of the acetonide group using TFA to give 14 . This intermediate was protected with the TIPDS group to afford 15, using the conditions reported by Sierzchala et al., ${ }^{47}$ before removing the benzyl protecting group by hydrogenation. Once deprotected, the resulting acid $\mathbf{1 6}$ was then coupled to the pantetheine tail 17 (synthesised in 2 steps from pantothenic acid following a modified version of the procedure of Virga et $a .^{48}$ ) using EDC in moderate yield before removing the silyl protecting group. Due to the concern of the strong basicity of the $\mathrm{F}^{-}$ion from TBAF causing unwanted side reactions, ${ }^{49,50}$ ammonium fluoride was successfully applied to the silyl deprotection instead. ${ }^{51}$ The synthetic route was repeated with the initial conversion of the adenosine $6^{\prime}-\mathrm{OH}$ to an $-\mathrm{NH}_{2}$ group, using conditions developed by Yao et $a^{52}$ to afford $\mathbf{1 9}$, which was then coupled with linker $\mathbf{1 2}$ to give $\mathbf{2 0}$. The addition 
of the silyl protecting group to $\mathbf{2 1}$ to give $\mathbf{2 2}$ was found to be higher yielding when the conditions developed by Kim et al. ${ }^{53}$ were used. Hydrogenation to give $\mathbf{2 3}$ was again followed by coupling of $\mathbf{1 7}$ to afford 24, which was finally deprotected to give 3. During the development of the synthetic routes to $\mathbf{1}$ and $\mathbf{3}$, compounds $\mathbf{2}$ and $\mathbf{4}$, lacking the pantetheine tail, were also synthesised as controls (Scheme 2).

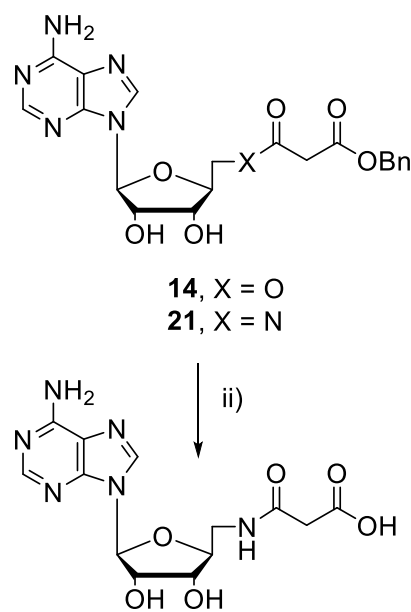

4

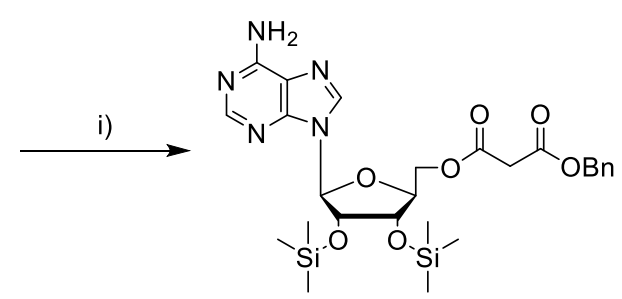

25

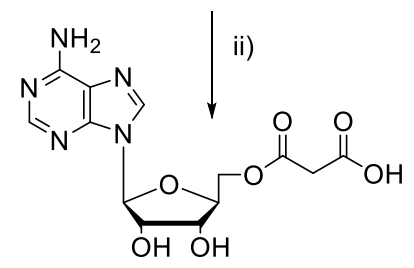

2

Scheme 2: Synthesis of 2 and 4. Conditions: (i) 2.5 eq. $\mathrm{TMSCl}_{1} 3.0$ eq. $\mathrm{NEt}_{3}, \mathrm{CH}_{2} \mathrm{Cl}_{2}, \mathrm{RT}, 16 \mathrm{~h}, 56 \%$; (ii) $10 \mathrm{~mol} \%$ $\mathrm{Pd} / \mathrm{C}, \mathrm{MeOH}, \mathrm{RT}$, 5-6 h, 2: 87\%, 4: 98\%.

A modified procedure was developed for the synthesis of the extended linker analogues $\mathbf{5}$ and $\mathbf{6}$. In order to develop a more convergent approach, we wished to first prepare the TIDPS protected adenosine 26. Direct protection of adenosine (27) with $\mathrm{TIDPSCl}_{2}$ gave the $3^{\prime}, 5^{\prime}$-protected adenosine derivative, as expected: unfortunately, attempted isomerisation to the required $2^{\prime}, 3^{\prime}$-protected adenosine 26, using previously reported conditions ${ }^{54,55}$ was unsuccessful. We therefore elected to carry out initial protection of the $5^{\prime}$ position with the dimethoxytrityl (DMTr) protecting group, utilising the conditions developed by Matulic-Adamic et $a^{56}$ to afford 28. Subsequent silyl addition to give 29 was followed by trityl group removal with acetic acid, ${ }^{57}$ which gave $\mathbf{2 6}$ (Scheme 3 ). The esterification proceeded well with linker lengths of 1 carbon $(\mathbf{1 2})^{44} 2$ carbons $(\mathbf{3 0})^{58}$ and 3 carbons (34), ${ }^{58}$ giving $\mathbf{1 5}$, 31 and 35 respectively. The conversions of 31 to 32 , and 35 to 36, were performed as before. As the coupling of $\mathbf{3 2}$ to the pantetheine tail $\mathbf{1 7}$ using EDC, and the equivalent coupling of $\mathbf{3 6}$ with 17, gave products (33 and $\mathbf{3 7}$ ) that were difficult to purify, the silyl group was removed directly from each intermediate using ammonium fluoride to afford 5 and 6 respectively. 


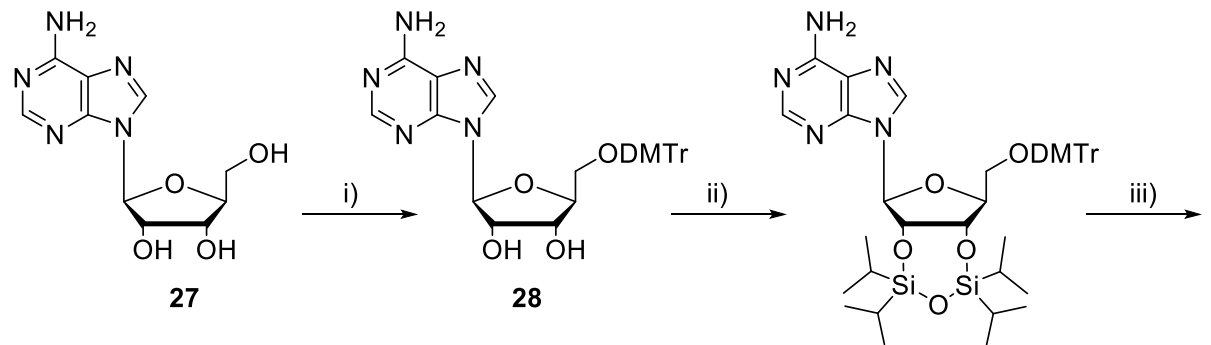

29

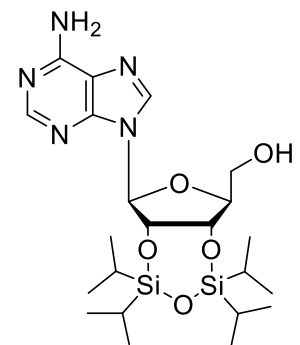

26

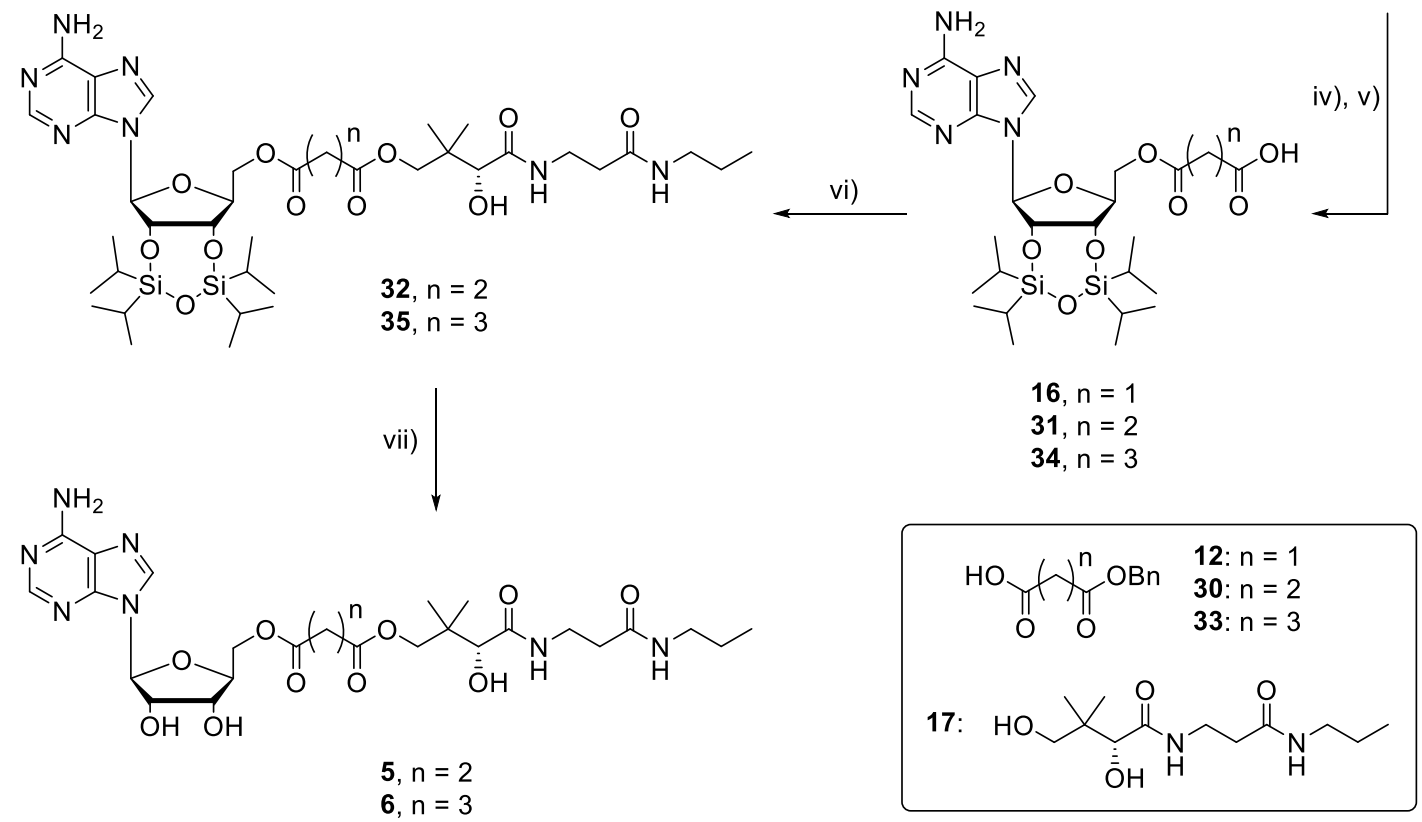

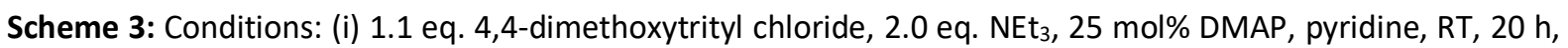
34\%; (ii) 1.2 eq. TIPDSCl$_{2}, 2.5$ eq. imidazole, 25 mol\% DMAP, DMF, RT, 17 h, 61\%; (iii) 80\% AcOH, RT, 4 h, 74\%; (iv) 2.0 eq. alcohol, 1.3 eq. DCC, 25 mol\% DMAP, $\mathrm{CH}_{2} \mathrm{Cl}_{2}, \mathrm{RT}, 18 \mathrm{~h}$, alcohol 12 gave 15: 90\%; alcohol 30 gave 31 : 85\%; alcohol 34 gave 35 34\%; (v) 10 mol\% Pd/C, MeOH, RT, 3-5 h, 32: 97\%, 36: 97\%; (vi) 1.0 eq. 17, 1.2 eq. EDC, 1.2 eq. DMAP, RT, 18 h; (vii) 8.5 eq. $\mathrm{NH}_{4} \mathrm{~F}, \mathrm{MeOH}, \mathrm{RT}, 1.5$ h, 5: $1 \%$ over two steps; 6 : 3\% over two steps.

\subsection{Role of the pantetheine tail}

As our docking studies and the crystal structure of the Aurora A kinase/CoA complex had indicated the importance of the pantetheine tail, we wished to explore whether simplified analogues with shorter tail structures would also be effective inhibitors. Structural analogues of CoA bearing modified pantetheine tails have not previously been reported as enzyme inhibitors. However, pantetheine analogues are important lead compounds as inhibitors of pantothenate kinase, ${ }^{59,60}$ a key enzyme in the biosynthesis of CoA which is a validated target for antimicrobial chemotherapy. A range of structural mimics of pantetheine side chains have therefore been developed, ${ }^{45,48,61,62}$ and these were used as a starting point for the present study. Prior to the synthesis of further analogues, alternative 
esterification conditions for the coupling of the tail to the linker moiety were investigated, and it was found that the use of DIC as the coupling agent gave the highest yields. These conditions were then applied to the syntheses of truncated analogues $\mathbf{7}, \mathbf{8}$, and $\mathbf{9}$ (Scheme 4). Alcohol $\mathbf{4 0}$ was synthesised according to the literature procedure. ${ }^{63}$ Alcohol $\mathbf{4 2}$ was synthesised by the ring opening of Dpantolactone with $N$-methyl- $\beta$-aminopropionamide under basic conditions.

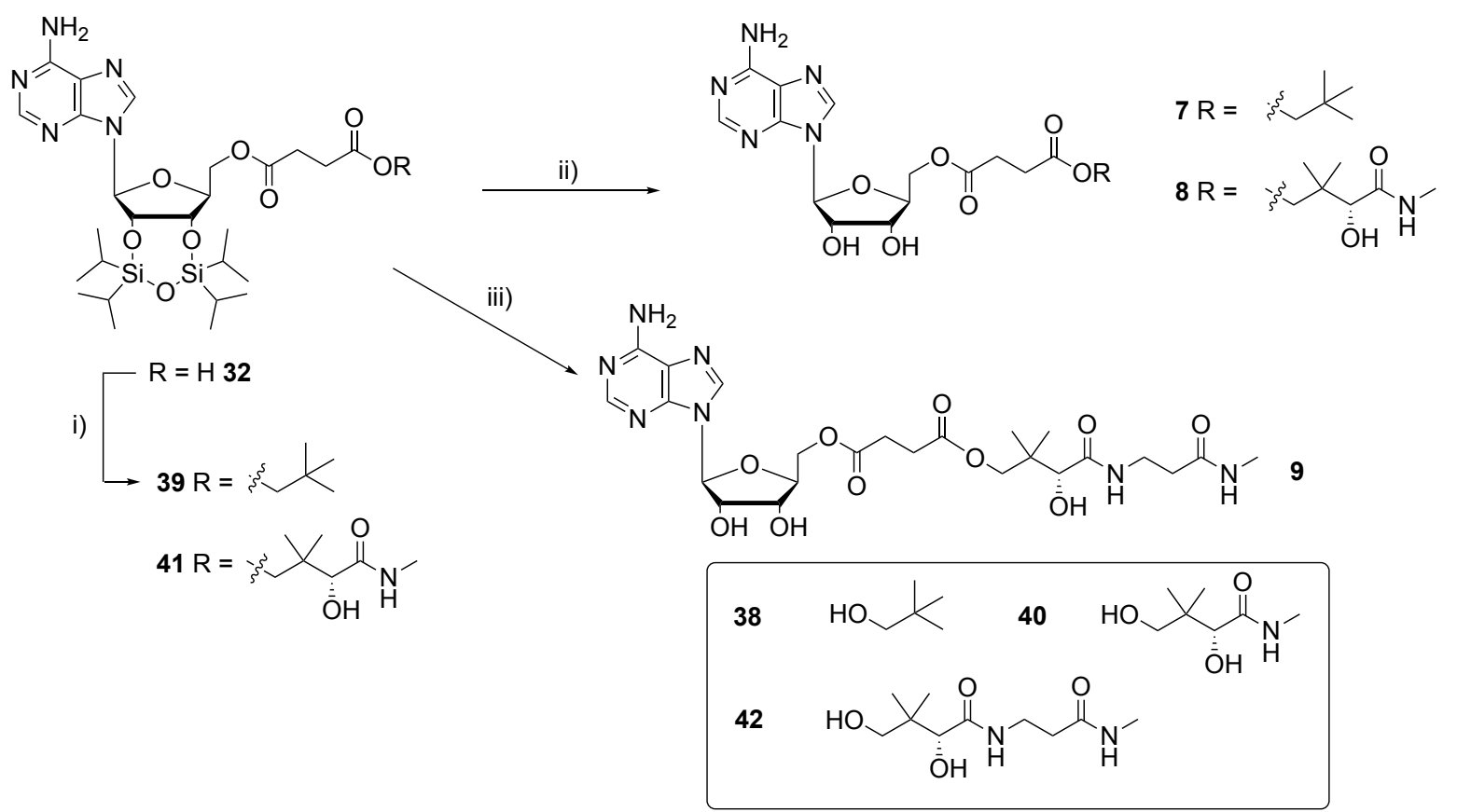

Scheme 4: Conditions: (i) 1.5 eq. DIC, 25 mol\% DMAP, $\mathrm{CH}_{2} \mathrm{Cl}_{2}, \mathrm{RT}, 20 \mathrm{~h}, 1.5$ eq. alcohol. 38 gave 39: 66\%, 40 gave 41: 46\%; (ii) 8.5 eq. $\mathrm{NH}_{4} \mathrm{~F}, \mathrm{MeOH}, \mathrm{RT}, 1.5$ h, 7: 59\%, 8: 34\%; (iii) 1.5 eq. DIC, 25 mol\% DMAP, $\mathrm{CH}_{2} \mathrm{Cl}_{2}, \mathrm{RT}, 20$ h, 1.5 eq. 42 , then 8.5 eq. $\mathrm{NH}_{4} \mathrm{~F}, \mathrm{MeOH}, \mathrm{RT}, 1.5 \mathrm{~h}, 18 \%$ over 2 steps.

Compound $\mathbf{1 0}$ was synthesised using alcohol $\mathbf{4 3}$ (the oxidised dimer of pantothenic acid) which gave a mixture of mono-substitution and the adenosine dimer $(\mathbf{4 4}, \mathbf{4 5})$, the crude material was directly treated with TBAF deprotection which enabled isolation of the dimer (46). The disulfide bond was then cleaved using TCEP to give 10 (Scheme 5). 


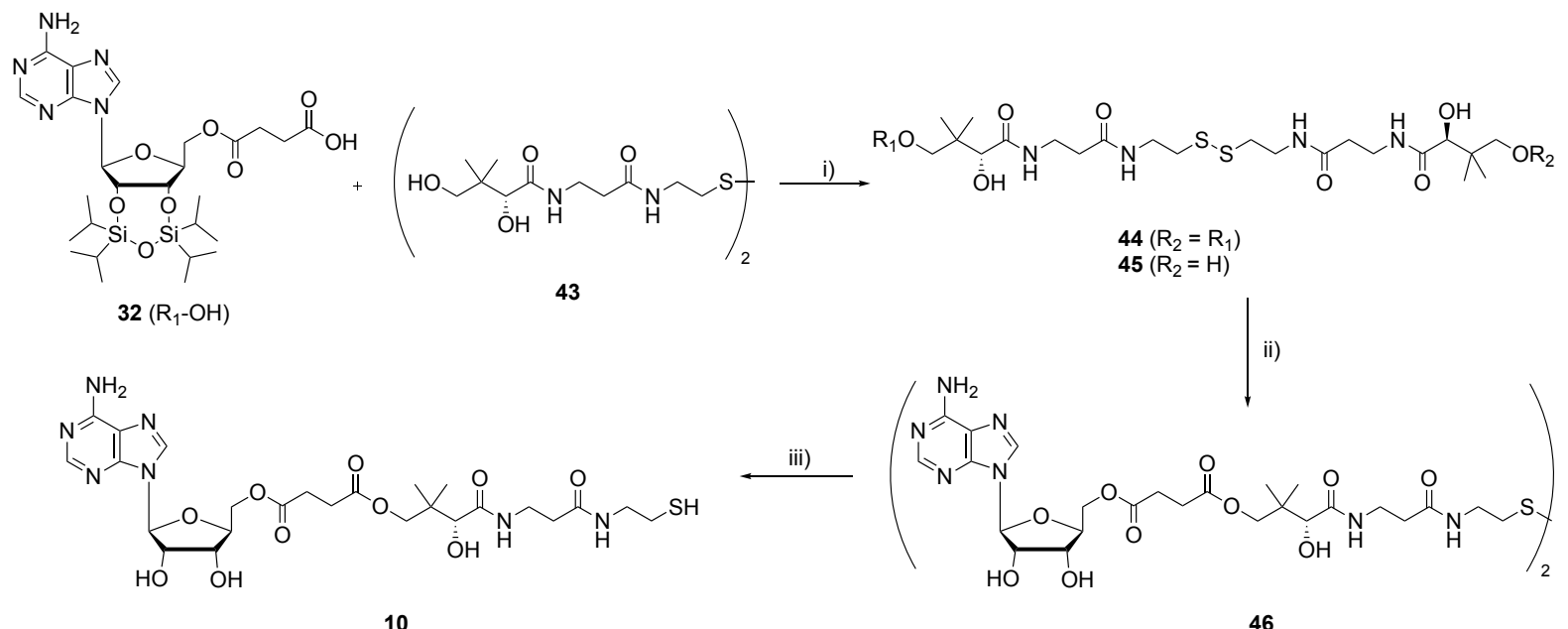

Scheme 5: Conditions: (i) 1.5 eq. DIC, 25 mol\% DMAP, $\mathrm{CH}_{2} \mathrm{Cl}_{2}, \mathrm{RT}, 20 \mathrm{~h}, 1.5$ eq alcohol 43 gave a mixture of singly added (44) and doubly added (45) products, which was then treated (ii) with 8.5 eq. $\mathrm{NH}_{4} \mathrm{~F}, \mathrm{MeOH}, \mathrm{RT}, 1.5$ $h$ to give 46: 4\% over 2 steps; (iii) 10.0 eq. TCEP, $\mathrm{H}_{2} \mathrm{O}$, MeCN, RT, 1 h, 10: $78 \%$.

\subsection{Biochemical and docking studies of Aurora A kinase inhibitors}

With our analogues in hand we measured the $\mathrm{IC}_{50}$ values against Aurora $A$ using a radioactive filterbinding assay (Table 2).

Table $2 \mathrm{IC}_{50}$ values against Aurora A.

\begin{tabular}{cc}
\hline Compound & Aurora A activity $\left(\mathrm{IC}_{50}, \mu \mathrm{M}\right)$ \\
\hline $\mathbf{1}$ & 1300 \\
$\mathbf{2}$ & 420 \\
$\mathbf{4}$ & 1100 \\
$\mathbf{5}$ & 910 \\
$\mathbf{6}$ & 497 \\
$\mathbf{7}$ & 542 \\
$\mathbf{8}$ & 430 \\
$\mathbf{9}$ & 1300 \\
10 & 1400 \\
CoA & 83 \\
dpCoA & 5 \\
desulfo-CoA & 17 \\
\hline
\end{tabular}

aFor this assay, CoA was used as a control and values are represented as the mean of at least two independent determinations and are within $\pm 30 \%$. ${ }^{b}$ dpCoA is an analogue of CoA lacking the $3^{\prime}$-phosphatte. ${ }^{c}$ desulfo-CoA is an analogue lacking the $-\mathrm{SH}$ group at the end of the pantetheine tail (data from reference 19)

Comparison of the $\mathrm{IC}_{50}$ values allows us to define some of the features of these analogues that are important for Aurora A kinase inhibition. Analogues with ester linkages, such as 2, showed a two-fold 
improvement in activity over equivalent analogues with amide linkages, e.g. 4. Secondly, the use of a longer linker $(\mathbf{5}, \mathbf{6})$ led to a two-fold increase in activity compared to the initial dicarbonyl analogue $\mathbf{1}$, indicating that in this case, the succinic or glutaric acid dicarbonyl moieties are better isosteres of the pyrophosphate group in CoA. Unfortunately, the docking results for these compounds are very similar to those obtained for $\mathbf{1}$ and do not explain the increase in potency. For both analogues, due to the flexibility of the tail and the linker, there is no clear preference to either binding mode available for the tail.

It is also clear that the terminal-SH group plays a crucial role in the recognition of CoA and analogues by Aurora A kinase. Comparison of the $\mathrm{IC}_{50}$ of analogue 10 with those for $\mathrm{dpCoA}$ and desulfo-CoA suggests that the presence of the -SH group is more important in determining the binding to Aurora $\mathrm{A}$ kinase than the presence of the $3^{\prime}$-phosphate or the pyrophosphate moieties. This is consistent with our recent discovery ${ }^{31}$ that Aurora $A$ is specifically CoAlated by the formation of a covalent bond between the -SH group and Cys290. In order to establish if a similar covalent interaction was present with appropriate CoA analogues, the activity assay of $\mathbf{1 0}$ was run in both the presence and absence of DTT. The inhibition of Aurora A by $\mathbf{1 0}$ was found to be DTT dependent, with the $\mathrm{IC}_{50}$ value for DTT present $731 \mu \mathrm{M}$ and the $\mathrm{IC}_{50}$ value for no DTT present $117 \mu \mathrm{M}$.

In the absence of a terminal -SH group, analogue 7 , with the shortest truncated tail structure, was found to be the most active. Comparison of the docking of $\mathbf{7}$ into the binding site of our model, Cys290modified crystal structure of the active Aurora A kinase (Section 2.1) with the binding of the other truncated structures $\mathbf{8}$ and $\mathbf{9}$ provides some insight into these results (Figure 3). Analogue $\mathbf{7}$ can be seen to fit perfectly in the binding site between the hinge region and the activation loop (Figure 3a) and therefore has good activity towards the kinase. By contrast, the longer pantetheine tail moieties of analogues $\mathbf{8}$ and $\mathbf{9}$ extend towards the back pocket of the kinase and thus cannot interact with either residues Thr 288 or Cys290 in the kinase activation loop, nor with the Gly-rich loop (Figure 3b, 3c). Additional analysis of the docking of 7 (Figure 3d)) highlights the possibility of extending the length of $\mathbf{7}$ by the addition of a group with the potential to interact with a cysteine residue within the active site to increase the activity of this analogue against Aurora A. 

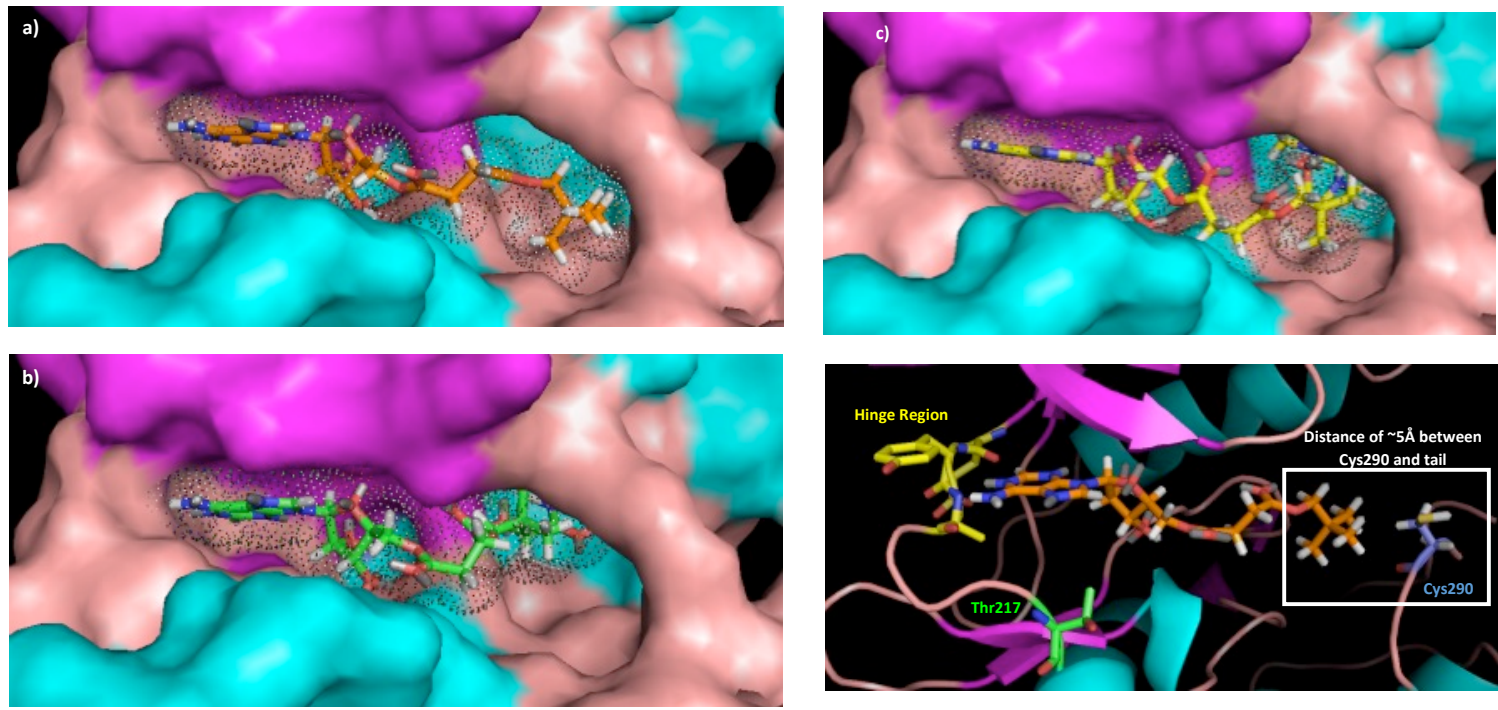

Figure 3: Comparison of the docking of three tested analogues: a) 7, b) $\mathbf{8}$ and c) $\mathbf{9}$ d) Docking of $\mathbf{7}$ in the Aurora A active site. This figure highlights the possibility of extending the tail when designing further analogues.

\section{Conclusion}

In this preliminary study, we have shown that understanding the structural basis for the unprecedented and highly selective inhibition of Aurora A kinase by $\mathrm{CoA}$ is an effective starting point for the design of inhibitors. We report the docking of CoA into the active site of a model structure of the active form of $\mathrm{CoA}$, and have compared this with our recently published Aurora A kinase-CoA complex structure. ${ }^{31}$ We have then used the model structure as the basis for docking studies.

We have designed and synthesised a series of simplified CoA analogues to probe the structural requirements for the inhibition of Aurora A. In particular, our results highlight the importance of targeting interactions with the Cys290 residue in the kinase activation loop, and also illustrate that quite simple dicarbonyl isosteres can be useful mimics of the pyrophosphate group of CoA. Of our synthesised compounds, analogue $\mathbf{1 0}$ has the best $\mathrm{IC}_{50}$ value against Aurora $\mathrm{A}$. It has improved drug likeness when compared to the parent molecule: the molecular weight has been reduced and the charged pyrophosphate group has been replaced with a neutral dicarbonyl linker, which should allow improved cell permeability. The interaction between the terminal -SH and Aurora A was found to be crucially important for activity, probably due to the formation of a disulfide bond formed between the terminal $-\mathrm{SH}$ of $\mathrm{COA}$ and the $-\mathrm{SH}$ of the cysteine residue contained in the activation loop. In the absence of the terminal $-\mathrm{SH}$ analogue $\mathbf{7}$ with the shortest pantetheine tail shows the best Aurora $\mathrm{A}$ activity, and we have rationalised this observation by docking studies. Further improvements in activity could come from truncated pantetheine tail analogues with $-\mathrm{SH}$ termini to interact with Cys290. These analogues would have a lower molecular weight and improved drug likeness when 
compared to CoA. In addition, extensive literature on alternative adenosine groups may allow this headgroup to be simplified. Combining these modifications with analogues that have an additional Thr217 interaction (such as H-bonding or a phosphate mimic) could further increase the potency of these compounds towards Aurora A. Finally, the majority of Aurora kinase inhibitors so far reported are competitive inhibitors which mimic the binding of ATP at the active site. ${ }^{3,8,9,10,11}$ This work opens up the possibility for the design of selective, irreversible (Type IV) covalent inhibitors based on analogue 10, which have the potential both to block the ATP active site and to alter the Aurora A conformation to prevent the binding of TPX2. Inhibitors with such a novel mode of action thus represent an important new paradigm for developing anticancer agents with reduced off-target side effects.

\section{Experimental}

\subsection{Chemistry}

${ }^{1} \mathrm{H}$ and ${ }^{13} \mathrm{C}$ NMR spectra of novel compounds can be found in the supporting information along with full spectral assignments. Known compounds $12,{ }^{44} \mathbf{1 7},{ }^{48} \mathbf{1 9},{ }^{64} \mathbf{2 8},{ }^{65} \mathbf{3 0},{ }^{66} \mathbf{3 4}{ }^{58}$ and $\mathbf{4 0}{ }^{63}$ were synthesised according to the published literature procedures. Data and procedures for these compounds can be found in the supporting information.

\subsubsection{General}

Unless otherwise stated, all reagents were purchased from commercial suppliers and used without purifications. All reactions were carried under Argon. Thin layer chromatography (TLC) was performed on aluminium backed Sigma-Aldrich TLC plates with $F_{254}$ fluorescent indicator, flash column chromatography was carried out using silica gel $(43-60 \mu \mathrm{m})$ supplied by Merck. Optical rotations were recorded using a Perkin Elmer 343 polarimeter and specific rotations are reported in $10^{-1} \mathrm{deg} \mathrm{cm}^{2} \mathrm{~g}^{-1}$ and concentrations (c) are reported as $10 \mathrm{mg} \mathrm{ml}^{-1}$. Melting points were recorded on a Gallenkamp Hot Stage apparatus and are uncorrected. Infrared spectra were recorded using a Perkin Elmer $100 \mathrm{FT}-\mathrm{IR}$ spectrometer and adsorption maxima are reported in wavenumbers $\left(\mathrm{cm}^{-1}\right)$. Electron ionisation (EI) and chemical ionisation ( $\mathrm{Cl}$ ) massed were determined using Thermo Finnigan MAT900 XP instrumentation. Electrospray ionisation (ESI) accurate mass was determined using Waters LCT Premier XE instrumentation. ${ }^{1} \mathrm{H}$ NMR and ${ }^{13} \mathrm{C}$ NMR spectra were obtained on 300,500 and $600 \mathrm{MHz}$ AMX Bruker Spectrometers using deuterated chloroform $\left(\mathrm{CDCl}_{3}\right)$, dimethylsulfoxide $\left(\mathrm{d}_{6}-\mathrm{DMSO}\right)$ and methanol $\left(\mathrm{CD}_{3} \mathrm{OD}\right)$ as solvents (as stated).

\subsubsection{Experimental Procedures and Data}


((3aS,4S,6S,6aS)-6-(6-Amino-9H-purin-9-yl)-2,2-dimethyltetrahydrofuro[3,4-d][1,3]dioxol-4-

yl)methyl benzyl malonate (13). To a solution of acid 12 (3.79 g, $19.5 \mathrm{mmol}$ ) and protected adenosine 11 (3.00 g, $9.77 \mathrm{mmol})$ in $\mathrm{CH}_{2} \mathrm{Cl}_{2}$ (30 mL) was added DCC (2.54 g, $\left.12.7 \mathrm{mmol}\right)$ and DMAP (297 mg, 2.44 mmol). The reaction was stirred at RT for $19 \mathrm{~h}$. The insoluble dicyclohexylurea was removed by filtration, washed with $\mathrm{CH}_{2} \mathrm{Cl}_{2}$ and the filtrate concentrated in vacuo. Purification using flash column chromatography (Gradient: $2-3 \% \mathrm{MeOH} / \mathrm{CH}_{2} \mathrm{Cl}_{2}$ ) gave $13(2.47 \mathrm{~g}, 52 \%)$ as a colourless gum; $\mathrm{R}_{\mathrm{f}} 0.51$ $\left(5 \% \mathrm{MeOH} / \mathrm{CH}_{2} \mathrm{Cl}_{2}\right.$ ); $[\alpha]_{D}{ }^{20}-21.2$ (c 0.7, $\mathrm{MeOH}$ ); IR v $\max$ (solution in $\left.\mathrm{CH}_{2} \mathrm{Cl}_{2}\right) 3320\left(\mathrm{NH}_{2}\right), 3060$ - 2930 (CH), $1730(\mathrm{C}=0)$ and $1600(\mathrm{C}=\mathrm{C}) \mathrm{cm}^{-1} ;{ }^{1} \mathrm{H} \mathrm{NMR}\left(600 \mathrm{MHz}, \mathrm{CDCl}_{3}\right) \delta 8.32(1 \mathrm{H}, \mathrm{s}), 7.89(1 \mathrm{H}, \mathrm{s}), 7.37-7.28(5 \mathrm{H}$, m), $6.27(2 \mathrm{H}, \mathrm{br}), 6.10(1 \mathrm{H}, \mathrm{s}), 5.42(1 \mathrm{H}, \mathrm{d}, J=6.4 \mathrm{~Hz}), 5.15(1 \mathrm{H}, \mathrm{d}, J=12.0 \mathrm{~Hz}), 5.13(1 \mathrm{H}, \mathrm{d}, J=12.0 \mathrm{~Hz})$, $5.04(1 \mathrm{H}, \mathrm{dd}, J=6.4$ and $3.4 \mathrm{~Hz}), 4.50-4.29(3 \mathrm{H}, \mathrm{m}), 3.41(1 \mathrm{H}, \mathrm{d}, J=10.0 \mathrm{~Hz}), 3.38(1 \mathrm{H}, \mathrm{d}, J=10.0 \mathrm{~Hz})$, $1.60(3 \mathrm{H}, \mathrm{s})$ and $1.38(3 \mathrm{H}, \mathrm{s}) ;{ }^{13} \mathrm{C}$ NMR $\left(150 \mathrm{MHz} \mathrm{CDCl}_{3}\right) \delta 166.3,166.0,155.9,153.3,149.3,139.8$, 135.2, 128.5 (overlapping signals), 120.3, 102.7, 90.9, 84.8, 84.3, 81.6, 67.5, 64.9, 41.3, 27.3 and 25.5; HRMS calc'd for $\mathrm{C}_{23} \mathrm{H}_{26} \mathrm{~N}_{5} \mathrm{O}_{7}$ expected 484.1832, found 484.1861.

\section{((2S,3R,4S,5S)-5-(6-Amino-9H-purin-9-yl)-3,4-dihydroxytetrahydrofuran-2-yl)methylbenzyl}

malonate (14). To a suspension of adenosine analogue $13(3.90 \mathrm{~g}, 8.07 \mathrm{mmol})$ in $\mathrm{H}_{2} \mathrm{O}(120 \mathrm{~mL})$ at $0{ }^{\circ} \mathrm{C}$ was added TFA $(30 \mathrm{~mL})$. The solution was stirred at $0{ }^{\circ} \mathrm{C}$ for $30 \mathrm{~min}$ before stirring at RT for $4 \mathrm{~h}$. The reaction mixture was then concentrated in vacuo. Purification using flash column chromatography (Gradient: $2 \%, 5 \%$ then $7.5 \% \mathrm{MeOH} / \mathrm{CH}_{2} \mathrm{Cl}_{2}$ ) gave 14 (2.86 g, 80\%) as an off-white solid; $\mathrm{R}_{\mathrm{f}} 0.37$ (10\% $\mathrm{MeOH} / \mathrm{CH}_{2} \mathrm{Cl}_{2}$ ); mp $131-133^{\circ} \mathrm{C} ;[\alpha]_{D}{ }^{20}-40.5$ (c 0.4, MeOH); IR $v_{\max }$ (solid) 3320 (NH), 3280 (NH), 3120 $(\mathrm{OH}), 3050-2980(\mathrm{CH}), 1680(\mathrm{C}=\mathrm{O})$ and $1600(\mathrm{C}=\mathrm{C}) \mathrm{cm}^{-1} ;{ }^{1} \mathrm{H}$ NMR $\left(600 \mathrm{MHz}, \mathrm{CD}_{3} \mathrm{OD}\right) \delta 8.34(1 \mathrm{H}, \mathrm{s})$, $8.27(1 \mathrm{H}, \mathrm{s}), 7.38-7.24(5 \mathrm{H}, \mathrm{m}), 6.03(1 \mathrm{H}, \mathrm{d}, J=4.9 \mathrm{~Hz}), 5.14(2 \mathrm{H}$, apparent s), $4.66(1 \mathrm{H}, \mathrm{t}, J=4.9 \mathrm{~Hz})$, $4.44(2 \mathrm{H}, \mathrm{dd}, J=4.0 \mathrm{~Hz}, 2.5 \mathrm{~Hz}), 4.33(1 \mathrm{H}, \mathrm{t}, J=4.9 \mathrm{~Hz})$ and $4.27(1 \mathrm{H}, \mathrm{q}, J=4.5 \mathrm{~Hz}) ;{ }^{13} \mathrm{C} \mathrm{NMR}(150 \mathrm{MHz}$, $\mathrm{CD}_{3} \mathrm{OD}$ ) $\delta 168.0,167.9,155.1,150.4,142.3,136.9,129.5$ (overlapping signals), 120.5, 90.3, 83.6, 75.3, 71.6, 68.2, 65.5 and 41.7; HRMS calc'd for $\mathrm{C}_{20} \mathrm{H}_{22} \mathrm{~N}_{5} \mathrm{O}_{7}$ expected 444.1519, found 444.1509. Note: (CO) $\mathrm{CH}_{2}(\mathrm{CO})$ signal is not seen in ${ }^{1} \mathrm{H}$ NMR as it is exchangeable with NMR solvent.

\section{((5aS,6S,8S,8aS)-8-(6-Amino-9H-purin-9-yl)-2,2,4,4-tetraisopropyltetrahydrofuro[3,4-}

f][1,3,5,2,4]trioxadisilepin-6-yl)methyl benzyl malonate (15). Route 1: To adenosine diol 14 (2.20 g, $4.96 \mathrm{mmol}$ ) in anhydrous pyridine $(27 \mathrm{~mL})$ was added 1,3-dichloro-1,1,3,3-triisopropylsiloxane (1.75 $\mathrm{mL}, 5.46 \mathrm{mmol}$ ). The reaction was stirred at RT for $18 \mathrm{~h}$. The reaction mixture was concentrated in vacuo. Purification using flash column chromatography (Gradient: 0 - 3\% $\mathrm{MeOH} / \mathrm{CH}_{2} \mathrm{Cl}_{2}$ ) gave $15(1.55$ g, 45\%) as an off-white solid. Route 2: To a solution of alcohol 26 (650 mg, $1.28 \mathrm{mmol}$ ) and acid 12 (497 mg, $2.56 \mathrm{mmol}$ ) in $\mathrm{CH}_{2} \mathrm{Cl}_{2}(8 \mathrm{~mL}$ ) was added DCC (333 mg, $1.66 \mathrm{mmol}$ ) and DMAP (39 mg, 0.32 mmol). The reaction was stirred at RT for $18 \mathrm{~h}$. The insoluble dicyclohexylurea was removed by filtration, washed with $\mathrm{CH}_{2} \mathrm{Cl}_{2}$ and the filtrate concentrated in vacuo. Purification using flash column 
chromatography (Gradient: $0-3 \% \mathrm{MeOH} / \mathrm{CH}_{2} \mathrm{Cl}_{2}$ ) gave 15 (790 mg, $90 \%$ ) as an off-white solid. $\mathrm{R}_{\mathrm{f}} 0.73$ $\left(10 \% \mathrm{MeOH} / \mathrm{CH}_{2} \mathrm{Cl}_{2}\right.$ ); mp $132-135^{\circ} \mathrm{C} ;[\alpha]_{D}^{20}-19.5$ (c 0.6, MeOH); IR $v_{\max }$ (solid) $3280(\mathrm{NH}), 3150(\mathrm{NH})$, $2940-2870(\mathrm{CH}), 1740(\mathrm{C}=\mathrm{O}) 1690(\mathrm{C}=\mathrm{O})$ and $1570(\mathrm{C}=\mathrm{C}) \mathrm{cm}^{-1} ;{ }^{1} \mathrm{H}$ NMR $\left(600 \mathrm{MHz}, \mathrm{CDCl}_{3}\right) \delta 8.32(1 \mathrm{H}$, s), $7.98(1 \mathrm{H}, \mathrm{s}), 7.36-7.29(5 \mathrm{H}, \mathrm{m}), 5.99(1 \mathrm{H}, \mathrm{d}, J=3.4 \mathrm{~Hz}), 5.91(2 \mathrm{H}, \mathrm{br}), 5.17(2 \mathrm{H}$, apparent s), 5.09 $(1 \mathrm{H}, \mathrm{dd}, J=4.9$ and $3.4 \mathrm{~Hz}), 4.72(1 \mathrm{H}, \mathrm{dd}, J=6.4$ and $4.9 \mathrm{~Hz}), 4.53(1 \mathrm{H}, \mathrm{dd}, J=12.0$ and $3.2 \mathrm{~Hz}), 4.41$ $(1 \mathrm{H}, \mathrm{dd}, J=12.0$ and $5.2 \mathrm{~Hz}), 4.33(1 \mathrm{H}, \mathrm{ddd}, J=6.4,5.2$ and $3.2 \mathrm{~Hz}), 3.46(2 \mathrm{H}$, apparent s) and $1.10-$ $0.99(28 \mathrm{H}, \mathrm{m}) ;{ }^{13} \mathrm{CNMR}\left(150 \mathrm{MHz}, \mathrm{CDCl}_{3}\right) \delta 166.2,155.7,153.2,149.6,140.2,135.2,128.6$ (overlapping signals), $120.5,90.6,81.7,75.7,72.6,67.5,64.6,41.4,17.4$ and $13.5 ; \mathrm{HRMS}$ calc'd for $\mathrm{C}_{32} \mathrm{H}_{48} \mathrm{O}_{8} \mathrm{~N}_{5} \mathrm{Si}_{2}$ expected 686.3041 , found 686.3073 .

\section{3-(((5aS,6S,8S,8aS)-8-(6-Amino-9H-purin-9-yl)-2,2,4,4-tetraisopropyltetrahydrofuro[3,4-}

$f][1,3,5,2,4]$ trioxadisilepin-6-yl)methoxy)-3-oxopropanoic acid (16). To protected adenosine analogue 15 (1.40 g, $2.04 \mathrm{mmol})$ and $\mathrm{Pd} / \mathrm{C}(10 \mathrm{wt} \%, 55 \mathrm{mg}, 0.51 \mathrm{mmol}$ ) was added $\mathrm{MeOH}(5 \mathrm{~mL})$ before evacuating and filling the flask with hydrogen. The reaction was stirred at RT under a hydrogen atmosphere for $5 \mathrm{~h}$. The reaction mixture was filtered through Celite ${ }^{\circledR}$, washed with $\mathrm{MeOH}$ and the filtrate concentrated in vacuo to give $16(860 \mathrm{mg}, 71 \%)$ as an off-white solid; $R_{f} 0.13$ (8\% $\mathrm{MeOH} / \mathrm{CH}_{2} \mathrm{Cl}_{2}$ ); mp $159-161^{\circ} \mathrm{C} ;[\alpha]_{\mathrm{D}}^{20}-17.5$ (c 0.2, MeOH); IR $v_{\max }$ (solid) $3320(\mathrm{NH}), 3280$ (NH), 3170 (OH), $2940-2870(\mathrm{CH}), 1750$ (C=O), $1670(\mathrm{C}=0)$ and 1620 (C=C) cm ${ }^{-1} ;{ }^{1} \mathrm{H}$ NMR (600 MHz, $d_{6}$-DMSO) $\delta$ $12.91(1 \mathrm{H}, \mathrm{br}), 8.38(1 \mathrm{H}, \mathrm{s}), 8.14(1 \mathrm{H}, \mathrm{s}), 7.36(1 \mathrm{H}, \mathrm{br}), 6.00(1 \mathrm{H}, \mathrm{d}, J=5.2 \mathrm{~Hz}), 5.17(1 \mathrm{H}, \mathrm{t}, J=5.2 \mathrm{~Hz})$, $4.74(1 \mathrm{H}, \mathrm{t}, J=4.7 \mathrm{~Hz}), 4.39(1 \mathrm{H}, \mathrm{dd}, J=11.9$ and $4.8 \mathrm{~Hz}), 4.28(1 \mathrm{H}, \mathrm{dd}, J=11.9$ and $5.6 \mathrm{~Hz}), 4.19(1 \mathrm{H}$, $\mathrm{q}, J=4.8 \mathrm{~Hz}), 3.40\left(2 \mathrm{H}\right.$, apparent s) and $1.09-0.92(28 \mathrm{H}, \mathrm{m}) ;{ }^{13} \mathrm{C} \mathrm{NMR}\left(150 \mathrm{MHz}, d_{6}\right.$-DMSO) $\delta$ 167.9, $166.8,156.2,152.8,149.3,139.7,119.1,81.7,81.7,74.8,72.3,63.9,41.4,17.2$ and 12.5; HRMS calc'd for $\mathrm{C}_{25} \mathrm{H}_{42} \mathrm{O}_{8} \mathrm{~N}_{5} \mathrm{Si}_{2}$ expected 596.2572 , found 596.2566

\section{((5aS,6S,8S,8aS)-8-(6-Amino-9H-purin-9-yl)-2,2,4,4-tetraisopropyltetrahydrofuro[3,4-}

$f][1,3,5,2,4]$ trioxadisilepin-6-yl)methyl

((R)-3-hydroxy-2,2-dimethyl-4-oxo-4-((3-0xo-3(propylamino)propyl)amino)butyl) malonate (18). To a solution of acid 16 (700 mg, $1.17 \mathrm{mmol}$ ) and alcohol 17 (337 mg, $1.30 \mathrm{mmol})$ in $\mathrm{CH}_{2} \mathrm{Cl}_{2}(30 \mathrm{~mL})$ at $0{ }^{\circ} \mathrm{C}$ was added EDC (271 mg, $\left.1.42 \mathrm{mmol}\right)$ and DMAP (173 mg, $1.42 \mathrm{mmol}$ ). The reaction was stirred at RT for $16 \mathrm{~h}$. The reaction mixture was diluted with $\mathrm{CH}_{2} \mathrm{Cl}_{2}(20 \mathrm{~mL})$ and washed with $5 \%$ citric acid solution $(40 \mathrm{~mL})$, then sat. $\mathrm{NaHCO}_{3}$ solution (40 $\mathrm{mL})$. The organic layer was dried $\left(\mathrm{MgSO}_{4}\right)$, filtered and concentrated in vacuo. Purification using flash column chromatography (Gradient: $2 \%, 5 \%$ then $7.5 \% \mathrm{MeOH} / \mathrm{CH}_{2} \mathrm{Cl}_{2}$ ) gave $18(240 \mathrm{mg}, 25 \%$ ) as an offwhite solid; $\mathrm{R}_{\mathrm{f}} 0.28\left(10 \% \mathrm{MeOH} / \mathrm{CH}_{2} \mathrm{Cl}_{2}\right.$ ); $\mathrm{mp} 84-86^{\circ} \mathrm{C}$; $[\alpha]_{\mathrm{D}}{ }^{20}+2.4$ (c $\left.0.2, \mathrm{MeOH}\right) ; \mathrm{IR} v_{\max }$ (solid) 3310 (NH), $3280(\mathrm{NH}), 2940-2860(\mathrm{CH}), 1730$ (C=O), 1640 (C=O) and $1600(\mathrm{C}=\mathrm{C}) \mathrm{cm}^{-1} ;{ }^{1} \mathrm{H}$ NMR (600 MHz, $\left.\mathrm{CDCl}_{3}\right) \delta 8.30(1 \mathrm{H}, \mathrm{s}), 8.09(1 \mathrm{H}, \mathrm{s}), 7.52(1 \mathrm{H}, \mathrm{t}, J=6.0 \mathrm{~Hz}), 6.75(1 \mathrm{H}, \mathrm{d}, J=4.5 \mathrm{~Hz}), 6.07(2 \mathrm{H}, \mathrm{br}), 6.04(1 \mathrm{H}$, s). $5.73(1 \mathrm{H}, \mathrm{t}, J=5.6 \mathrm{~Hz}), 4.97(1 \mathrm{H}, \mathrm{d}, J=3.7 \mathrm{~Hz}), 4.64(1 \mathrm{H}, \mathrm{dd}, J=12.4$ and $2.3 \mathrm{~Hz}), 4.49-4.42(2 \mathrm{H}$, 
m), $4.40-4.35(2 \mathrm{H}, \mathrm{m}), 4.15(1 \mathrm{H}, \mathrm{d}, J=4.9 \mathrm{~Hz}), 3.84(1 \mathrm{H}, \mathrm{d}, J=10.9 \mathrm{~Hz}), 3.67-3.59(1 \mathrm{H}, \mathrm{m}), 3.56-$ $3.49(1 \mathrm{H}, \mathrm{m}), 3.48(2 \mathrm{H}$, apparent s), $3.29-3.16(2 \mathrm{H}, \mathrm{m}), 2.51-2.37(2 \mathrm{H}, \mathrm{m}), 1.74-1.56(2 \mathrm{H}, \mathrm{m})$ and $1.17-0.89(37 \mathrm{H}, \mathrm{m}) ;{ }^{13} \mathrm{C}$ NMR $\left(150 \mathrm{MHz}, \mathrm{CDCl}_{3}\right) \delta 172.5,171.4,166.7,166.0,155.3,153.5,149.0$, $138.4,119.6$, 91.0, 81.1, 76.3, 73.8, 71.8, 71.5, 63.0, 41.4, 41.3, 38.5, 35.6, 35.2, 22.9, 21.9, 18.9, 14.5, 13.7 and 13.0; HRMS calc'd for $\mathrm{C}_{37} \mathrm{H}_{64} \mathrm{O}_{11} \mathrm{~N}_{7} \mathrm{Si}_{2}$ expected 838.4202, found 838.4238.

((2S,3R,4S,5S)-5-(6-Amino-9H-purin-9-yl)-3,4-dihydroxytetrahydrofuran-2-yl)methyl

$((R)-3-$ hydroxy-2,2-dimethyl-4-oxo-4-((3-oxo-3-(propylamino)propyl)amino)butyl) malonate (1). To a solution of protected CoA analogue $18(70.0 \mathrm{mg}, 0.08 \mathrm{mmol})$ in $\mathrm{MeOH}(2 \mathrm{~mL})$ was added ammonium fluoride $(25 \mathrm{mg}, 0.68 \mathrm{mmol})$ and the reaction was stirred at RT for $1.5 \mathrm{~h}$. The reaction mixture was concentrated in vacuo. Purification using flash column chromatography (Gradient: $5 \%, 10 \%$ then $12.5 \% \mathrm{MeOH} / \mathrm{CH}_{2} \mathrm{Cl}_{2}$ ) gave 1 (20 mg, 42\%) as a colourless oil; $\mathrm{R}_{\mathrm{f}} 0.40\left(15 \% \mathrm{MeOH} / \mathrm{CH}_{2} \mathrm{Cl}_{2}\right) ; \mathrm{mp} 96$ - 98 ${ }^{\circ} \mathrm{C} ;[\alpha]_{\mathrm{D}}^{20}+54.5$ (c 0.1, MeOH); IR $v_{\max }$ (oil) $3320(\mathrm{NH}), 3280(\mathrm{NH}), 3080(\mathrm{OH}), 2960-2880(\mathrm{CH}), 1730$ $(\mathrm{C}=\mathrm{O}), 1640(\mathrm{C}=\mathrm{O})$ and $1570(\mathrm{C}=\mathrm{C}) \mathrm{cm}^{-1}$; ${ }^{1} \mathrm{H}$ NMR $\left(600 \mathrm{MHz}, \mathrm{CD}_{3} \mathrm{OD}\right) \delta 8.28(1 \mathrm{H}, \mathrm{s}), 8.21(1 \mathrm{H}, \mathrm{s}), 6.02$ $(1 \mathrm{H}, \mathrm{d}, J=4.5 \mathrm{~Hz}), 4.72(1 \mathrm{H}, \mathrm{t}, J=4.5 \mathrm{~Hz}), 4.45(2 \mathrm{H}, \mathrm{dd}, J=14.5$ and $4.0 \mathrm{~Hz}), 4.39(1 \mathrm{H}, \mathrm{t}, J=5.0 \mathrm{~Hz}), 4.29$ $-4.26(1 \mathrm{H}, \mathrm{m}), 4.11(1 \mathrm{H}, \mathrm{d}, J=10.5 \mathrm{~Hz}), 3.95(1 \mathrm{H}, \mathrm{d}, J=10.5 \mathrm{~Hz}), 3.86(1 \mathrm{H}, \mathrm{s}), 3.50-3.39(2 \mathrm{H}, \mathrm{m}), 3.10$ $(2 \mathrm{H}, \mathrm{t}, J=6.5 \mathrm{~Hz}), 2.40(2 \mathrm{H}, \mathrm{t}, J=6.8 \mathrm{~Hz}), 1.54-1.45(2 \mathrm{H}, \mathrm{m}), 0.95(3 \mathrm{H}, \mathrm{s})$ and $0.92-0.88(6 \mathrm{H}, \mathrm{m}) ;{ }^{13} \mathrm{C}$ NMR $\left(150 \mathrm{MHz}, \mathrm{CD}_{3} \mathrm{OD}\right) \delta$ 175.3, 173.6, 168.2, 168.0, 157.6, 153.4, 150.6, 142.0, 121.0, 90.1, 83.4, 79.3, 75.7, 72.7, 71.7, 65.9, 42.4, 41.6, 39.6, 36.4, 23.6, 20.7, 20.6 and 11.7; HRMS calc'd for $\mathrm{C}_{25} \mathrm{H}_{38} \mathrm{~N}_{7} \mathrm{O}_{10}$ expected 596.2680, found 596.2684. Note: (CO) $\mathrm{CH}_{2}(\mathrm{CO})$ signal is not seen in ${ }^{1} \mathrm{H}$ NMR as it is exchangeable with NMR solvent.

Benzyl

3-((((3aS,4S,6S,6aS)-6-(6-amino-9H-purin-9-yl)-2,2-dimethyltetrahydrofuro[3,4d][1,3]dioxol-4-yl)methyl)amino)-3-oxopropanoate (20). To a solution of amine 19 (3.50 g, 11.4 $\mathrm{mmol})$ and acid $12(4.42 \mathrm{~g}, 22.8 \mathrm{mmol})$ in $\mathrm{CH}_{2} \mathrm{Cl}_{2}(35 \mathrm{~mL})$ was added DCC (2.96 g, $\left.14.8 \mathrm{mmol}\right)$ and DMAP (347 mg, $2.85 \mathrm{mmol}$ ). The reaction was stirred at RT for $21 \mathrm{~h}$. The insoluble dicyclohexylurea was removed by filtration, washed with $\mathrm{CH}_{2} \mathrm{Cl}_{2}$ and the filtrate concentrated in vacuo. Purification using flash column chromatography (Gradient: $0-3 \% \mathrm{MeOH} / \mathrm{CH}_{2} \mathrm{Cl}_{2}$ ) gave $20(4.26 \mathrm{~g}, 78 \%$ ) as a white gum; $\mathrm{R}_{\mathrm{f}} 0.63\left(10 \% \mathrm{MeOH} / \mathrm{CH}_{2} \mathrm{Cl}_{2}\right.$ ); mp $77-79^{\circ} \mathrm{C} ;[\alpha]_{\mathrm{D}}^{20}-68.2$ (c 0.5, MeOH); IR $v_{\max }$ (solution in $\mathrm{CH}_{2} \mathrm{Cl}_{2}$ ) 3320 (NH), $3180(\mathrm{NH}), 3060-2920(\mathrm{CH}), 1730$ (C=O), 1640 (C=O) and $1590(\mathrm{C}=\mathrm{C}) \mathrm{cm}^{-1} ;{ }^{1} \mathrm{H}$ NMR $(600 \mathrm{MHz}$, $\left.\mathrm{CDCl}_{3}\right) \delta 8.70(1 \mathrm{H}, \mathrm{d}, J=7.9 \mathrm{~Hz}), 8.26(1 \mathrm{H}, \mathrm{s}), 7.85(1 \mathrm{H}, \mathrm{s}), 7.28-7.20(5 \mathrm{H}, \mathrm{m}), 6.12(2 \mathrm{H}, \mathrm{br}), 5.8(1 \mathrm{H}, \mathrm{d}$, $J=4.9 \mathrm{~Hz}), 5.19(1 \mathrm{H}, \mathrm{dd}, J=6.0$ and $4.9 \mathrm{~Hz}), 5.13(1 \mathrm{H}, \mathrm{d}, J=12.5 \mathrm{~Hz}), 5.10(1 \mathrm{H}, \mathrm{d}, J=12.5 \mathrm{~Hz}), 4.89(1 \mathrm{H}$, $\mathrm{dd}, J=6.0$ and $2.0 \mathrm{~Hz}), 4.49(1 \mathrm{H}, \mathrm{q}, J=2.0 \mathrm{~Hz}), 4.17(1 \mathrm{H}, \mathrm{ddd}, J=14.4,8.8$ and $2.5 \mathrm{~Hz}), 3.48(2 \mathrm{H}$, apparent s), $3.29(1 \mathrm{H}, \mathrm{dt}, J=14.4$ and $2.5 \mathrm{~Hz}), 1.62(3 \mathrm{H}, \mathrm{s})$ and $1.36(3 \mathrm{H}, \mathrm{d}) ;{ }^{13} \mathrm{C} \mathrm{NMR}\left(150 \mathrm{MHz}, \mathrm{CDCl}_{3}\right)$ $\delta 168.4,166.0,156.1,152.6,148.8,140.6,135.2,128.6$ (overlapping signals), 121.2, 114.8, 92.8, 83.7, 
82.4, 81.7, 67.4, 42.9, 41.4, 27.6 and 25.3; HRMS calc'd for $\mathrm{C}_{23} \mathrm{H}_{19} \mathrm{~N}_{6} \mathrm{O}_{6}$ expected 483.1992, found 483.1992.

Benzyl

3-((( (2S,3R,4S,5S)-5-(6-amino-9H-purin-9-yl)-3,4-dihydroxytetrahydrofuran-2-

yl)methyl)amino)-3-oxopropanoate (21). To a cooled suspension of protected adenosine analogue 20 $(4.20 \mathrm{~g}, 8.71 \mathrm{mmol})$ in $\mathrm{H}_{2} \mathrm{O}(130 \mathrm{~mL})$ was added TFA $(33 \mathrm{~mL})$ and the reaction stirred at $0{ }^{\circ} \mathrm{C}$ for $30 \mathrm{~min}$ before warming to RT for $3 \mathrm{~h}$. The reaction mixture was then concentrated in vacuo. Purification using flash column chromatography (Gradient: $2 \%, 5 \%$ then $7.5 \% \mathrm{MeOH} / \mathrm{CH}_{2} \mathrm{Cl}_{2}$ ) gave $21(3.47 \mathrm{~g}, 90 \%$ ) as an off-white solid; $\mathrm{R}_{\mathrm{f}} 0.39$ ( $10 \% \mathrm{MeOH} / \mathrm{CH}_{2} \mathrm{Cl}_{2}$ ); mp $113-116^{\circ} \mathrm{C}$; $[\alpha]_{\mathrm{D}}{ }^{20}-55.2$ (c $0.5, \mathrm{MeOH}$ ); IR $v_{\max }$ (solid) $3350(\mathrm{NH}), 3290(\mathrm{NH}), 3100(\mathrm{OH}), 3070-2900(\mathrm{CH}), 1710(\mathrm{C}=\mathrm{O}), 1650(\mathrm{C}=\mathrm{O})$ and $1580(\mathrm{C}=\mathrm{C}) \mathrm{cm}^{-1} ;{ }^{1} \mathrm{H}$ NMR $\left(600 \mathrm{MHz}, d_{6}\right.$-DMSO) $\delta 8.51(1 \mathrm{H}, \mathrm{s}), 8.45(1 \mathrm{H}, \mathrm{t}, J=5.8 \mathrm{~Hz}), 8.33(1 \mathrm{H}, \mathrm{s}), 8.20(2 \mathrm{H}, \mathrm{br}), 7.37-7.29$ $(5 \mathrm{H}, \mathrm{m}), 5.90(1 \mathrm{H}, \mathrm{d}, J=6.0 \mathrm{~Hz}), 5.51(2 \mathrm{H}, \mathrm{br}), 5.11(2 \mathrm{H}$, apparent s), $4.64(1 \mathrm{H}, \mathrm{t}, J=6.0 \mathrm{~Hz}), 4.09(1 \mathrm{H}, \mathrm{t}$, $J=4.7 \mathrm{~Hz}), 3.97(1 \mathrm{H}, \mathrm{dt}, J=5.8$ and $4.7 \mathrm{~Hz}), 3.50-3.38(2 \mathrm{H}, \mathrm{m})$ and $3.35(2 \mathrm{H}$, apparent $\mathrm{s}) ;{ }^{13} \mathrm{C} \mathrm{NMR}$ (150 MHz, $d_{6}$-DMSO) $\delta 167.8,165.4,153.9,149.7,148.9,141.3,135.9,128.4,128.0,127.8,119.2,87.7$, 83.5, 73.0, 71.2, 65.9, 42.4 and 41.2; $\mathrm{HRMS}$ calc'd for $\mathrm{C}_{20} \mathrm{H}_{23} \mathrm{O}_{6} \mathrm{~N}_{6}$ expected 443.1679, found 443.1672.

Benzyl 3-((((5aS,6S,8S,8aS)-8-(6-amino-9H-purin-9-yl)-2,2,4,4-tetraisopropyltetrahydrofuro[3,4$f][1,3,5,2,4]$ trioxadisilepin-6-yl)methyl)amino)-3-oxopropanoate (22). To adenosine diol 21 (350 mg, $0.79 \mathrm{mmol}$ ) in anhydrous DMF ( $3 \mathrm{~mL}$ ) was added imidazole $(135 \mathrm{mg}, 1.98 \mathrm{mmol})$ and DMAP $(24 \mathrm{mg}$, $0.20 \mathrm{mmol}$ ). Then, 1,3-dichloro-1,1,3,3-triisopropyldisiloxane (303 $\mu \mathrm{L}, 0.95 \mathrm{mmol}$ ) was added dropwise. The reaction was stirred at RT for $17 \mathrm{~h}$. The reaction was concentrated in vacuo. The resulting residue was dissolved in $\mathrm{CH}_{2} \mathrm{Cl}_{2}(10 \mathrm{~mL})$ and washed with sat. $\mathrm{NaHCO}_{3}$ solution $(10 \mathrm{~mL})$. The aqueous layer was extracted with $\mathrm{CH}_{2} \mathrm{Cl}_{2}(2 \times 10 \mathrm{~mL})$. The combined organics washed with brine $(10 \mathrm{~mL})$, dried $\left(\mathrm{MgSO}_{4}\right)$, filtered and concentrated in vacuo. Purification using flash column chromatography (Gradient: $0-3 \% \mathrm{MeOH}$ in $\mathrm{CH}_{2} \mathrm{Cl}_{2}$ ) gave 22 (330 mg, 61\%) as an off-white solid; $\mathrm{R}_{\mathrm{f}}$ $0.62\left(10 \% \mathrm{MeOH} / \mathrm{CH}_{2} \mathrm{Cl}_{2}\right.$ ); mp $66-68{ }^{\circ} \mathrm{C} ;[\alpha]_{\mathrm{D}}{ }^{20}-81.3$ (c 0.2, MeOH); IR $v_{\max }$ (solid) $3290(\mathrm{NH}), 3210$ (NH), $2950-2870(\mathrm{CH}), 1740$ (C=O), 1640 (C=O) and $1580(\mathrm{C}=\mathrm{C}) \mathrm{cm}^{-1} ;{ }^{1} \mathrm{H}$ NMR $\left(600 \mathrm{MHz}, \mathrm{CDCl}_{3}\right) \delta 8.72$ $(1 \mathrm{H}, \mathrm{d}, J=6.8 \mathrm{~Hz}), 8.33(1 \mathrm{H}, \mathrm{s}), 7.89(1 \mathrm{H}, \mathrm{s}), 7.38-7.29(5 \mathrm{H}, \mathrm{m}), 5.96(2 \mathrm{H}, \mathrm{br}), 5.79(1 \mathrm{H}, \mathrm{d}, J=7.0 \mathrm{~Hz})$, $5.16(2 \mathrm{H}$, apparent s), $5.03(1 \mathrm{H}, \mathrm{dd}, J=7.0$ and $5.0 \mathrm{~Hz}), 4.53(1 \mathrm{H}, \mathrm{dd}, J=5.0$ and $2.3 \mathrm{~Hz}), 4.37(1 \mathrm{H}, \mathrm{q}, J$ $=3.0 \mathrm{~Hz}), 4.14(1 \mathrm{H}, \mathrm{ddd}, J=14.5,8.8$ and $3.0 \mathrm{~Hz}), 3.47(1 \mathrm{H}, \mathrm{d}, J=15.0 \mathrm{~Hz}), 3.40(1 \mathrm{H}, \mathrm{d}, J=15.0 \mathrm{~Hz})$, $3.31(1 \mathrm{H}, \mathrm{dt}, J=14.3$ and $3.0 \mathrm{~Hz})$ and $1.13-0.87(28 \mathrm{H}, \mathrm{m}) ;{ }^{13} \mathrm{C} \mathrm{NMR}\left(150 \mathrm{MHz}, \mathrm{CDCl}_{3}\right) \delta$ 168.0, 165.6, $155.8,152.5,149.2,141.1,135.3,128.6$ (overlapping signals), 121.3, 90.9, 85.8, 75.4, 73.6, 67.4, 47.8, 41.3, 17.5 and 13.2; HRMS calc'd for $\mathrm{C}_{32} \mathrm{H}_{49} \mathrm{O}_{7} \mathrm{~N}_{6} \mathrm{Si}_{2}$ expected 685.3201, found 685.3226.

3-((((5aS,6S,8S,8aS)-8-(6-Amino-9H-purin-9-yl)-2,2,4,4-tetraisopropyltetrahydrofuro[3,4-

$f][1,3,5,2,4]$ trioxadisilepin-6-yl)methyl)amino)-3-oxopropanoic acid (23). To protected adenosine 
analogue $22(800 \mathrm{mg}, 1.17 \mathrm{mmol}$ ) and $\mathrm{Pd} / \mathrm{C}(10 \mathrm{wt} \%, 31 \mathrm{mg}, 0.29 \mathrm{mmol})$ was added $\mathrm{MeOH}(3 \mathrm{~mL})$ before evacuating and filling the flask with hydrogen. The reaction was stirred at RT under a hydrogen atmosphere for $3 \mathrm{~h}$. The reaction mixture was filtered through Celite ${ }^{\circ}$, washed with $\mathrm{MeOH}$ and the filtrate concentrated in vacuo to give $23(666 \mathrm{mg}, 95 \%)$ as an off-white solid; $R_{f} 0.10$ (5\% $\mathrm{MeOH} / \mathrm{CH}_{2} \mathrm{Cl}_{2}$ ); mp $129-131^{\circ} \mathrm{C} ;[\alpha]_{D}^{20}-108.6$ (c 0.2, MeOH); IR $v_{\max }$ (solid) 3280 (NH), 3210 (NH), 3070 (OH), $2950-2870(\mathrm{CH}), 1700(\mathrm{C}=0), 1660(\mathrm{C}=0)$ and $1580(\mathrm{C}=\mathrm{C}) \mathrm{cm}^{-1} ;{ }^{1} \mathrm{H}$ NMR (600 MHz, $d_{6}$-DMSO) $\delta$ $8.66(1 \mathrm{H}, \mathrm{br}), 8.46(1 \mathrm{H}, \mathrm{s}), 8.16(1 \mathrm{H}, \mathrm{s}), 7.37(2 \mathrm{H}, \mathrm{br}), 5.94(1 \mathrm{H}, \mathrm{d}, J=6.4 \mathrm{~Hz}), 5.15(1 \mathrm{H}, \mathrm{dd}, J=6.4$ and $4.9 \mathrm{~Hz}), 4.59(1 \mathrm{H}, \mathrm{dd}, J=5.0$ and $3.2 \mathrm{~Hz}), 4.05(1 \mathrm{H}, \mathrm{dt}, J=5.0$ and $3.0 \mathrm{~Hz}) 3.50-3.40(2 \mathrm{H}, \mathrm{m}), 3.34(1 \mathrm{H}$, br), $3.11\left(2 \mathrm{H}\right.$, apparent s) and $1.13-0.83(28 \mathrm{H}, \mathrm{m}) ;{ }^{13} \mathrm{C}$ NMR $\left(150 \mathrm{MHz}, d_{6}\right.$-DMSO) $\delta 169.5,166.8$, $156.2,152.7,149.4,140.1,119.3,87.2,83.9,74.7,73.1,42.9,41.0,17.3$ and 13.2; HRMS calc'd for $\mathrm{C}_{25} \mathrm{H}_{43} \mathrm{O}_{7} \mathrm{~N}_{6} \mathrm{Si}_{2}$ expected 595.2732, found 595.2723.

(R)-3-Hydroxy-2,2-dimethyl-4-oxo-4-((3-oxo-3-(propylamino)propyl)amino)butyl 3((((5aS,6S,8S,8aS)-8-(6-amino-9H-purin-9-yl)-2,2,4,4-tetraisopropyltetrahydrofuro[3,4-

$f][1,3,5,2,4]$ trioxadisilepin-6-yl)methyl)amino)-3-oxopropanoate (24). To a solution of acid 23 (270 $\mathrm{mg}, 0.45 \mathrm{mmol})$ and alcohol 17 (140 mg, $0.54 \mathrm{mmol})$ in $\mathrm{CH}_{2} \mathrm{Cl}_{2}(13 \mathrm{~mL})$ at $0{ }^{\circ} \mathrm{C}$ was added EDC (104 mg, $0.54 \mathrm{mmol}$ ) and DMAP (66 mg, $0.54 \mathrm{mmol}$ ). The reaction was stirred at RT for $18 \mathrm{~h}$. The reaction mixture was diluted with $\mathrm{CH}_{2} \mathrm{Cl}_{2}(10 \mathrm{~mL})$ and washed with $5 \%$ citric acid solution $(10 \mathrm{~mL})$, then sat. $\mathrm{NaHCO}_{3}$ solution $(10 \mathrm{~mL})$. The organic layer was dried $\left(\mathrm{MgSO}_{4}\right)$, filtered and concentrated in vacuo. Purification using flash column chromatography (Gradient: $2 \%, 5 \%$ then 7.5\% $\mathrm{MeOH} / \mathrm{CH}_{2} \mathrm{Cl}_{2}$ ) gave 24 (51 mg, 14\%) as an off-white solid; $\mathrm{R}_{\mathrm{f}} 0.59\left(10 \% \mathrm{MeOH} / \mathrm{CH}_{2} \mathrm{Cl}_{2}\right.$ ); mp $106-107^{\circ} \mathrm{C}$; $[\alpha]_{\mathrm{D}}{ }^{20}-41.5$ (c 0.1 , $\mathrm{MeOH}$ ); IR $v_{\max }$ (solid) $3320(\mathrm{NH}), 3210(\mathrm{NH}), 3080(\mathrm{OH}), 2950-2870(\mathrm{CH}), 1740(\mathrm{C}=\mathrm{O}), 1640(\mathrm{C}=\mathrm{O})$ and $1590(\mathrm{C}=\mathrm{C}) \mathrm{cm}^{-1} ;{ }^{1} \mathrm{H} N M R\left(600 \mathrm{MHz}, \mathrm{CDCl}_{3}\right) \delta 8.97(1 \mathrm{H}, \mathrm{d}, J=8.2 \mathrm{~Hz}), 8.31(1 \mathrm{H}, \mathrm{s}), 7.90(1 \mathrm{H}, \mathrm{d}), 7.59(1 \mathrm{H}$, $\mathrm{t}, J=5.8 \mathrm{~Hz}), 6.40(2 \mathrm{H}, \mathrm{br}), 6.09(1 \mathrm{H}, \mathrm{br}), 5.80(1 \mathrm{H}, \mathrm{d}, J=6.6 \mathrm{~Hz}), 4.98(1 \mathrm{H}, \mathrm{dd}, J=6.6$ and $5.1 \mathrm{~Hz}), 4.48$ $(1 \mathrm{H}, \mathrm{dd}, J=5.1$ and $2.5 \mathrm{~Hz}), 4.37(1 \mathrm{H}, \mathrm{q}, J=2.5 \mathrm{~Hz}), 4.26(1 \mathrm{H}, \mathrm{d}, J=10.6 \mathrm{~Hz}), 4.14(1 \mathrm{H}, \mathrm{ddd}, J=14.4,9.1$ and $2.5 \mathrm{~Hz}), 4.02(1 \mathrm{H}, \mathrm{s}), 3.77(1 \mathrm{H}, \mathrm{d}, J=10.6 \mathrm{~Hz}), 3.68-3.60(1 \mathrm{H}, \mathrm{m}), 3.54-3.37(3 \mathrm{H}, \mathrm{m}), 3.29(1 \mathrm{H}$, $\mathrm{dt}, J=14.4$ and $2.5 \mathrm{~Hz}), 3.21-3.13(2 \mathrm{H}, \mathrm{m}), 2.51-2.39(2 \mathrm{H}, \mathrm{m}), 1.53-1.45(2 \mathrm{H}, \mathrm{m})$ and $1.12-0.86$ $(37 \mathrm{H}, \mathrm{m}) ;{ }^{13} \mathrm{C}$ NMR $\left(150 \mathrm{MHz}, \mathrm{CDCl}_{3}\right) \delta 173.1,171.4,168.0,166.1,156.7,152.4,148.9,140.8,121.1$, $91.0,85.6,75.6,74.3,73.5,71.8,42.6,41.5,41.1,38.3,36.1,35.5,22.8,21.8,19.7,17.5,12.9$ and 11.5; HRMS calc'd for $\mathrm{C}_{37} \mathrm{H}_{65} \mathrm{O}_{10} \mathrm{~N}_{8} \mathrm{Si}_{2}$ expected 837.4362, found 837.4365.

(R)-3-Hydroxy-2,2-dimethyl-4-oxo-4-((3-oxo-3-(propylamino)propyl)amino)butyl 3-((((2S,3R,4S,5S)5-(6-amino-9H-purin-9-yl)-3,4-dihydroxytetrahydrofuran-2-yl)methyl)amino)-3-oxopropanoate (3). To a solution of protected CoA analogue $24(35.0 \mathrm{mg}, 0.04 \mathrm{mmol})$ in $\mathrm{MeOH}(1 \mathrm{~mL})$ was added ammonium fluoride $(13 \mathrm{mg}, 0.36 \mathrm{mmol}$ ) and the reaction was stirred at RT for $1.5 \mathrm{~h}$. The reaction mixture was concentrated in vacuo. Purification using flash column chromatography (Gradient: 5\%, 
$10 \%$ then $15 \% \mathrm{MeOH} / \mathrm{CH}_{2} \mathrm{Cl}_{2}$ ) gave $3(8 \mathrm{mg}, 75 \%)$ as a colourless oil; $\mathrm{R}_{\mathrm{f}} 0.16\left(15 \% \mathrm{MeOH} / \mathrm{CH}_{2} \mathrm{Cl}_{2}\right) ; \mathrm{mp}$ $101-103^{\circ} \mathrm{C} ;[\alpha]_{D}{ }^{20}-38.5$ (c 0.1, MeOH); IR $v_{\max }$ (oil) $3310(\mathrm{NH}), 3280(\mathrm{NH}), 3180(\mathrm{OH}), 2960-2870$ $(\mathrm{CH}), 1730(\mathrm{C}=\mathrm{O}), 1640(\mathrm{C}=\mathrm{O})$ and $1600(\mathrm{C}=\mathrm{C}) \mathrm{cm}^{-1} ;{ }^{1} \mathrm{H}$ NMR $\left(600 \mathrm{MHz}, \mathrm{CD}_{3} \mathrm{OD}\right) \delta 8.26(1 \mathrm{H}, \mathrm{s}), 8.25(1 \mathrm{H}$, s), $5.92(1 \mathrm{H}, \mathrm{d}, J=6.2 \mathrm{~Hz}), 4.80(1 \mathrm{H}, \mathrm{t}, J=6.2 \mathrm{~Hz}), 4.25-4.18(2 \mathrm{H}, \mathrm{m}), 4.08(1 \mathrm{H}, \mathrm{d}, J=10.6 \mathrm{~Hz}), 3.94$ $(1 \mathrm{H}, \mathrm{d}, J=10.6 \mathrm{~Hz}), 3.86-3.81(2 \mathrm{H}, \mathrm{m}), 3.49-3.39(3 \mathrm{H}, \mathrm{m}), 3.35(2 \mathrm{H}$, apparent s), $3.12-3.08(2 \mathrm{H}, \mathrm{m})$, $2.42(2 \mathrm{H}, \mathrm{t}, J=6.7 \mathrm{~Hz}), 1.53-1.45(2 \mathrm{H}, \mathrm{m}), 0.96(3 \mathrm{H}, \mathrm{s}), 0.93(3 \mathrm{H}, \mathrm{s})$ and $0.90(3 \mathrm{H}, \mathrm{t}, J=7.4 \mathrm{~Hz}) ;{ }^{13} \mathrm{C}$ NMR (150 MHz, CD $\mathrm{OD}) \delta$ 176.1, 175.3, 169.4, 168.8, 157.5, 153.8, 150.4, 142.2, 121.1, 90.8, 85.5, $77.2,75.7,74.5,72.8,49.5,42.5,42.4,39.5,36.9,23.6,21.6,21.4$ and 11.7; HRMS calc'd for $\mathrm{C}_{25} \mathrm{H}_{39} \mathrm{~N}_{8} \mathrm{O}_{9}$ expected 595.2840 , found 595.2857 .

\section{((2S,3S,4S,5S)-5-(6-Amino-9H-purin-9-yl)-3,4-bis((trimethylsilyl)oxy)tetrahydrofuran-2-yl)methyl}

benzyl malonate (25). To a solution of adenosine diol 14 (800 mg, $1.80 \mathrm{mmol}^{\text {) and NEt }}$ (750 $\mu \mathrm{L}, 5.40$ mmol) in $\mathrm{CH}_{2} \mathrm{Cl}_{2}(9 \mathrm{~mL})$ at $0{ }^{\circ} \mathrm{C}$ was added $\mathrm{TMSCl}(570 \mu \mathrm{L}, 4.50 \mathrm{mmol})$ dropwise. The reaction was stirred at RT for $16 \mathrm{~h}$. The reaction mixture was filtered, washed with $\mathrm{Et}_{2} \mathrm{O}$ and the filtrate was concentrated in vacuo. Purification using flash column chromatography (Gradient: $50 \%$ then then $80 \%$ EtOAc/pet. ether) gave 25 (590 mg, 56\%) as an off-white solid; $R_{f} 0.57\left(100 \%\right.$ EtOAc); mp $103-105^{\circ} \mathrm{C}$; $[\alpha]_{D}^{20}-29.6$ (c 0.2, MeOH); IR $v_{\max }$ (solid) $3280(\mathrm{NH}), 3140(\mathrm{NH}), 2950-2900(\mathrm{CH}), 1730$ (C=O), 1680 $(\mathrm{C}=0)$ and $1600(\mathrm{C}=\mathrm{C}) \mathrm{cm}^{-1} ;{ }^{1} \mathrm{H} \mathrm{NMR}\left(600 \mathrm{MHz}, \mathrm{CDCl}_{3}\right) \delta 8.34(1 \mathrm{H}, \mathrm{s}), 7.96(1 \mathrm{H}, \mathrm{s}), 7.36-7.30(5 \mathrm{H}, \mathrm{m})$, $6.02(2 \mathrm{H}, \mathrm{br}), 5.90(1 \mathrm{H}, \mathrm{d}, J=4.1 \mathrm{~Hz}), 5.18(2 \mathrm{H}$, apparent s), $4.96(1 \mathrm{H}, \mathrm{t}, J=4.5 \mathrm{~Hz}), 4.54(1 \mathrm{H}, \mathrm{d}, J=3.8$ $\mathrm{Hz}), 4.42-4.35(2 \mathrm{H}, \mathrm{m}), 4.29(1 \mathrm{H}, \mathrm{q}, J=4.0 \mathrm{~Hz}), 3.48\left(2 \mathrm{H}\right.$, apparent s), $0.16(9 \mathrm{H}, \mathrm{s})$ and $0.01(9 \mathrm{H}, \mathrm{s}) ;{ }^{13} \mathrm{C}$ NMR (150 MHz, CDCl 3$) \delta 166.2,155.7,153.0,149.7,140.2,135.2,128.6$ (overlapping signals), 120.6, 89.8, 81.9, 74.2, 71.7, 67.5, 64.3, 41.4 and 0.26; HRMS calc'd for $\mathrm{C}_{26} \mathrm{H}_{38} \mathrm{~N}_{5} \mathrm{O}_{7} \mathrm{Si}$ expected 588.2310, found 588.2308 .

\section{3-(((2S,3R,4S,5S)-5-(6-Amino-9H-purin-9-yl)-3,4-dihydroxytetrahydrofuran-2-yl)methoxy)-3-}

oxopropanoic acid (2). To adenosine diol 25 (550 mg, $0.94 \mathrm{mmol}$ ) and Pd/C (10 wt\%, $25 \mathrm{mg}, 0.23$ $\mathrm{mmol}$ ) was added $\mathrm{MeOH}(3 \mathrm{~mL})$ before evacuating and filling the flask with hydrogen. The reaction was stirred at RT under a hydrogen atmosphere for $5 \mathrm{~h}$. The reaction mixture was filtered through Celite, washed with $\mathrm{MeOH}$ and the filtrate concentrated in vacuo. The resulting white solid was triturated in $\mathrm{Et}_{2} \mathrm{O}$ to give 2 (290 mg, 87\%) as an off-white solid; $\mathrm{R}_{\mathrm{f}} \mathrm{O} .60\left(10 \% \mathrm{MeOH} / \mathrm{CH}_{2} \mathrm{Cl}_{2}\right) ; \mathrm{mp} 148$ $150{ }^{\circ} \mathrm{C} ;[\alpha]_{D}{ }^{20}-33.3$ (c 0.7, MeOH); IR $v_{\max }$ (solid) $3310(\mathrm{NH}), 3220(\mathrm{NH}), 3120(\mathrm{OH}), 2980-2950(\mathrm{CH})$, $1740(\mathrm{C}=0), 1690$ (C=O) and $1600(\mathrm{C}=\mathrm{C}) \mathrm{cm}^{-1} ;{ }^{1} \mathrm{H}$ NMR (600 MHz, $d_{6}$-DMSO) $\delta 8.34(1 \mathrm{H}, \mathrm{s}), 8.15(1 \mathrm{H}, \mathrm{s})$, $7.32(2 \mathrm{H}, \mathrm{br}), 5.91(1 \mathrm{H}, \mathrm{d}, J=5.3 \mathrm{~Hz}), 4.64(1 \mathrm{H}, \mathrm{t}, J=5.3 \mathrm{~Hz}), 4.36(1 \mathrm{H}, \mathrm{dd}, J=12.0$ and $3.8 \mathrm{~Hz}), 4.25-$ $4.20(2 \mathrm{H}, \mathrm{m}), 4.11-4.07(1 \mathrm{H}, \mathrm{m})$ and $3.35\left(2 \mathrm{H}\right.$, apparent s); ${ }^{13} \mathrm{C} \mathrm{NMR} \mathrm{(150} \mathrm{MHz,} d_{6}$-DMSO) $\delta 168.2$, 167.3, 156.1, 152.7, 149.4, 139.8, 119.2, 87.7, 81.6, 72.9, 70.7, 64.0 and 42.1; HRMS calc'd for $\mathrm{C}_{13} \mathrm{H}_{16} \mathrm{O}_{7} \mathrm{~N}_{5}$ expected 354.1050, found 354.1037. 


\section{3-(((2S,3R,4S,5S)-5-(6-Amino-9H-purin-9-yl)-3,4-dihydroxytetrahydrofuran-2-yl)methyl)amino)-3-}

oxopropanoic acid (4). To protected adenosine diol 21 (100 mg, $0.23 \mathrm{mmol}$ ) and Pd/C (10 wt\%, $6 \mathrm{mg}$, $0.25 \mathrm{mmol})$ was added $\mathrm{MeOH}(2.5 \mathrm{~mL})$ before evacuating and filling the flask with hydrogen. The reaction was stirred at RT under a hydrogen atmosphere for $5 \mathrm{~h}$. The reaction mixture was filtered through Celite ${ }^{\circ}$, washed with $\mathrm{MeOH}$ and the filtrate concentrated in vacuo to give 4 ( $\left.80 \mathrm{mg}, 98 \%\right)$ as an off-white solid; $\mathrm{R}_{\mathrm{f}} 0.20\left(20 \% \mathrm{MeOH} / \mathrm{CH}_{2} \mathrm{Cl}_{2}\right)$; mp $105-108^{\circ} \mathrm{C}$; $[\alpha]_{D}{ }^{20}-71.4$ (c $\left.0.2, \mathrm{MeOH}\right) ; \mathrm{IR} v_{\max }$ (solid) $3290(\mathrm{NH}), 3260(\mathrm{NH}), 3210(\mathrm{OH}), 3180(\mathrm{OH}), 2960-2900(\mathrm{CH}), 1630(\mathrm{C}=\mathrm{O})$ and $1570(\mathrm{C}=\mathrm{C}) \mathrm{cm}^{-}$ 1; ${ }^{1} \mathrm{H}$ NMR $\left(600 \mathrm{MHz}, \mathrm{CD}_{3} \mathrm{OD}\right) \delta 8.49(1 \mathrm{H}, \mathrm{s}), 8.40(1 \mathrm{H}, \mathrm{s}), 6.02(1 \mathrm{H}, \mathrm{d}, J=5.7 \mathrm{~Hz}), 4.74(1 \mathrm{H}, \mathrm{t}, J=5.7 \mathrm{~Hz})$, $4.27(1 \mathrm{H}, \mathrm{dd}, J=5.1$ and $4.1 \mathrm{~Hz}), 4.19-4.14(1 \mathrm{H}, \mathrm{m}), 3.72(1 \mathrm{H}, \mathrm{dd}, J=14.2$ and $5.6 \mathrm{~Hz})$ and $3.56(1 \mathrm{H}$, dd, $J=14.2$ and $4.3 \mathrm{~Hz}) ;{ }^{13} \mathrm{C}$ NMR $\left(150 \mathrm{MHz}, \mathrm{CD}_{3} \mathrm{OD}\right) \delta 171.5,169.4,152.3,150.1,146.8,144.2,120.8$, 90.5, 85.4, 75.2, 72.6, 49.5 and 42.5; HRMS calc'd for $\mathrm{C}_{13} \mathrm{H}_{17} \mathrm{~N}_{6} \mathrm{O}_{6}$ expected 353.1210, found 353.1199. Note: $(\mathrm{CO}) \mathrm{CH}_{2}(\mathrm{CO})$ signal is not seen in ${ }^{1} \mathrm{H}$ NMR as it is exchangeable with NMR solvent.

\section{((5aS,6S,8S,8aS)-8-(6-Amino-9H-purin-9-yl)-2,2,4,4-tetraisopropyltetrahydrofuro[3,4-}

$f][1,3,5,2,4]$ trioxadisilepin-6-yl)methanol (26). Following a novel procedure. A solution of protected adenosine 29 ( $7.58 \mathrm{~g}, 9.34 \mathrm{mmol})$ in $80 \%$ acetic acid $(140 \mathrm{~mL})$ was stirred at RT for $4 \mathrm{~h}$. The reaction mixture was diluted with $\mathrm{H}_{2} \mathrm{O}$, cooled to $0{ }^{\circ} \mathrm{C}$ and taken to $\mathrm{pH}$ 8-9 with $2 \mathrm{M} \mathrm{NaOH}$ solution. The aqueous solution was extracted with $\mathrm{CH}_{2} \mathrm{Cl}_{2}(2 \times 500 \mathrm{~mL})$. The combined organic layers were dried $\left(\mathrm{MgSO}_{4}\right)$, filtered and concentrated in vacuo. Purification using flash column chromatography (Gradient: $0-3 \%$ $\mathrm{MeOH} / \mathrm{CH}_{2} \mathrm{Cl}_{2}$ ) gave 26 (3.50 mg, 74\%) as an off-white solid: $\mathrm{R}_{\mathrm{f}} 0.71\left(10 \% \mathrm{MeOH} / \mathrm{CH}_{2} \mathrm{Cl}_{2}\right) ; \mathrm{mp} 217-219$ ${ }^{\circ} \mathrm{C} ;[\alpha]_{\mathrm{D}}^{20}-94.1$ (c 0.1, MeOH); ${ }^{1} \mathrm{H}$ NMR $\left(600 \mathrm{MHz}, \mathrm{CDCl}_{3}\right) \delta 8.32(1 \mathrm{H}, \mathrm{s}), 7.83(1 \mathrm{H}, \mathrm{s}), 6.60(1 \mathrm{H}, \mathrm{br}), 5.94$ $(2 \mathrm{H}, \mathrm{br}), 5.79(1 \mathrm{H}, \mathrm{d}, J=7.7 \mathrm{~Hz}), 5.14(1 \mathrm{H}, \mathrm{dd}, J=7.7$ and $4.7 \mathrm{~Hz}), 4.68(1 \mathrm{H}, \mathrm{d}, J=4.7 \mathrm{~Hz}), 4.34(1 \mathrm{H}, \mathrm{d}, J$ $=1.3 \mathrm{~Hz}), 3.97(1 \mathrm{H}, \mathrm{dd}, J=13.0$ and $1.3 \mathrm{~Hz}), 3.74-3.67(1 \mathrm{H}, \mathrm{m})$ and $1.15-0.90(28 \mathrm{H}, \mathrm{m}) ;{ }^{13} \mathrm{C} \mathrm{NMR}(150$ $\left.\mathrm{MHz}, \mathrm{CDCl}_{3}\right) \delta 156.0,152.5,148.7,141.0,121.4,92.0,89.3,76.1,73.3,63.3,17.3$ and 13.4; HRMS calc'd for $\mathrm{C}_{22} \mathrm{H}_{40} \mathrm{O}_{5} \mathrm{~N}_{5} \mathrm{Si}_{2}$ expected 510.2568 , found 510.2564 . The data is in good agreement with the literature values. ${ }^{54}$

\section{9-((5aS,6S,8S,8aS)-8-((Bis(4-methoxyphenyl)(phenyl)methoxy)methyl)-2,2,4,4-} tetraisopropyltetrahydrofuro[3,4-f][1,3,5,2,4]trioxadisilepin-6-yl)-9H-purin-6-amine (29). To protected adenosine $28(8.70 \mathrm{~g}, 15.3 \mathrm{mmol})$ in anhydrous DMF $(60 \mathrm{~mL})$ was added imidazole $(2.60 \mathrm{~g}$, $38.3 \mathrm{mmol}$ ) and DMAP (466 mg, $3.83 \mathrm{mmol}$ ). Then, 1,3-dichloro-1,1,3,3-triisopropyldisiloxane (5.87 $\mathrm{mL}, 18.3 \mathrm{mmol}$ ) was added dropwise. The reaction was stirred at RT for $17 \mathrm{~h}$. The reaction was concentrated in vacuo. The resulting residue was dissolved in $\mathrm{CH}_{2} \mathrm{Cl}_{2}(400 \mathrm{~mL})$ and washed with sat. $\mathrm{NaHCO}_{3}$ solution $(400 \mathrm{~mL})$. The aqueous layer was extracted with $\mathrm{CH}_{2} \mathrm{Cl}_{2}(2 \times 300 \mathrm{~mL})$. The combined organics washed with brine $(400 \mathrm{~mL})$, dried $\left(\mathrm{MgSO}_{4}\right)$, filtered and concentrated in vacuo. Purification using flash column chromatography (Gradient: $0-2 \% \mathrm{MeOH}$ in $\mathrm{CH}_{2} \mathrm{Cl}_{2}$ ) gave $29(7.58 \mathrm{~g}, 61 \%$ ) as an 
off-white solid; $\mathrm{R}_{\mathrm{f}} 0.36\left(5 \% \mathrm{MeOH} / \mathrm{CH}_{2} \mathrm{Cl}_{2}\right.$ ); mp 67 - $69{ }^{\circ} \mathrm{C}$; $[\alpha]_{\mathrm{D}}{ }^{20}-11.2$ (c $0.6, \mathrm{MeOH}$ ); IR $v_{\max }$ (solid) $3330(\mathrm{NH}), 3280(\mathrm{NH}), 2950-2870(\mathrm{CH})$ and $1580(\mathrm{C}=\mathrm{C}) \mathrm{cm}^{-1} ;{ }^{1} \mathrm{H}$ NMR $\left(600 \mathrm{MHz}, \mathrm{CDCl}_{3}\right) \delta 8.31(1 \mathrm{H}, \mathrm{s})$, $8.11(1 \mathrm{H}, \mathrm{s}), 7.47-7.41(2 \mathrm{H}, \mathrm{m}), 7.36-7.31(4 \mathrm{H}, \mathrm{m}), 7.29-7.16(3 \mathrm{H}, \mathrm{m}), 6.83-6.78(4 \mathrm{H}, \mathrm{m}) .6 .10$ $(2 \mathrm{H}, \mathrm{br}), 6.06(1 \mathrm{H}, \mathrm{d}, J=4.7 \mathrm{~Hz}), 5.11(1 \mathrm{H}, \mathrm{d}, J=4.7 \mathrm{~Hz}), 4.78(1 \mathrm{H}, \mathrm{t}, J=4.7 \mathrm{~Hz}), 4.29-4.25(1 \mathrm{H}, \mathrm{m})$, $3.78(6 \mathrm{H}, \mathrm{s}), 3.50(1 \mathrm{H}, \mathrm{dd}, J=10.7$ and $3.4 \mathrm{~Hz}), 3.33(1 \mathrm{H}, \mathrm{dd}, J=10.7$ and $3.4 \mathrm{~Hz})$ and $1.13-0.92(28 \mathrm{H}$, m); ${ }^{13} \mathrm{C}$ NMR $\left(150 \mathrm{MHz}, \mathrm{CDCl}_{3}\right) \delta 158.7,155.7,153.2,150.0,144.8,139.3,135.9,130.1,128.3,128.2$, 127.0, 120.3, 113.5, 89.0, 86.6, 84.3, 76.2, 73.2, 63.3, 55.3, 17.2 and 13.7; HRMS calc'd for $\mathrm{C}_{43} \mathrm{H}_{58} \mathrm{O}_{7} \mathrm{~N}_{5} \mathrm{Si}_{2}$ expected 812.3875, found 812.3900 .

((5aS,6S,8S,8aS)-8-(6-Amino-9H-purin-9-yl)-2,2,4,4-tetraisopropyltetrahydrofuro[3,4-

$f][1,3,5,2,4]$ trioxadisilepin-6-yl)methyl benzyl succinate (31). To a solution of alcohol 26 (2.49 g, 4.89 $\mathrm{mmol})$ and protected succinic acid $30(1.22 \mathrm{~g}, 5.87 \mathrm{mmol})$ in $\mathrm{CH}_{2} \mathrm{Cl}_{2}(40 \mathrm{~mL})$ was added DCC $(1.27 \mathrm{~g}$, $6.36 \mathrm{mmol}$ ) and DMAP (149 $\mathrm{mg}, 1.22 \mathrm{mmol})$. The reaction was stirred at RT for $16 \mathrm{~h}$. The insoluble dicyclohexylurea was removed by filtration, washed with $\mathrm{CH}_{2} \mathrm{Cl}_{2}$. The organic filtrate was washed with sat. $\mathrm{NaHCO}_{3}$ solution $(3 \times 100 \mathrm{~mL})$, brine $(150 \mathrm{~mL})$, dried $\left(\mathrm{MgSO}_{4}\right)$, filtered and concentrated in vacuo. Purification using flash column chromatography (Gradient: 0 - 2\% $\mathrm{MeOH} / \mathrm{CH}_{2} \mathrm{Cl}_{2}$ ) gave $31(2.91 \mathrm{~g}, 85 \%$ ) as an off-white solid; $\mathrm{R}_{\mathrm{f}} 0.54\left(5 \% \mathrm{MeOH} / \mathrm{CH}_{2} \mathrm{Cl}_{2}\right.$ ); mp $135-137^{\circ} \mathrm{C}$; $[\alpha]_{\mathrm{D}}{ }^{20}-18.6$ (c 0.4, MeOH); IR $v_{\max }$ (solid) 3300 (NH), $3150(\mathrm{NH}), 2950-2870(\mathrm{CH}), 1740$ (C=O) and $1600(\mathrm{C}=\mathrm{C}) \mathrm{cm}^{-1} ;{ }^{1} \mathrm{H}$ NMR (600 MHz, $\left.\mathrm{CDCl}_{3}\right) \delta 8.32(1 \mathrm{H}, \mathrm{s}), 8.00(1 \mathrm{H}, \mathrm{s}), 7.38-7.29(5 \mathrm{H}, \mathrm{m}), 5.99(1 \mathrm{H}, \mathrm{d}, J=3.2), 5.88(2 \mathrm{H}, \mathrm{br}), 5.13(2 \mathrm{H}, \mathrm{s})$, $5.09(1 \mathrm{H}, \mathrm{dd}, J=4.8$ and $3.2 \mathrm{~Hz}), 4.70(1 \mathrm{H}, \mathrm{dd}, J=6.1$ and $4.8 \mathrm{~Hz}), 4.51-4.46(1 \mathrm{H}, \mathrm{m}), 4.36-4.31(2 \mathrm{H}$, m), $2.72-2.64(4 \mathrm{H}, \mathrm{m})$ and $1.11-0.97(28 \mathrm{H}, \mathrm{m}) ;{ }^{13} \mathrm{C} \mathrm{NMR}\left(150 \mathrm{MHz}, \mathrm{CDCl}_{3}\right) \delta 172.1,155.5,152.9$, 149.6, 139.7, 135.8, 128.5 (overlapping signals), 120.5, 90.5, 81.8, 75.8, 72.7, 66.8, 63.9, 29.1, 29.0, 17.3 and 13.6; HRMS calc'd for $\mathrm{C}_{33} \mathrm{H}_{50} \mathrm{O}_{8} \mathrm{~N}_{5} \mathrm{Si}_{2}$ expected 700.3198, found 700.3188 .

\section{4-(((5aS,6S,8S,8aS)-8-(6-Amino-9H-purin-9-yl)-2,2,4,4-tetraisopropyltetrahydrofuro[3,4-}

$f][1,3,5,2,4]$ trioxadisilepin-6-yl)methoxy)-4-oxobutanoic acid (32). To protected adenosine analogue $31(1.50 \mathrm{~g}, 2.15 \mathrm{mmol})$ and $\mathrm{Pd} / \mathrm{C}(10 \mathrm{wt} \%, 57 \mathrm{mg}, 0.54 \mathrm{mmol})$ was added $\mathrm{MeOH}(32 \mathrm{~mL})$ before evacuating and filling the flask with hydrogen. The reaction was stirred at RT under a hydrogen atmosphere for $5 \mathrm{~h}$. The reaction mixture was filtered through Celite, washed with $\mathrm{MeOH}$ and the filtrate concentrated in vacuo to give $32(1.28 \mathrm{~g}, 97 \%)$ as an off-white solid; $\mathrm{R}_{\mathrm{f}} 0.21\left(8 \% \mathrm{MeOH} / \mathrm{CH}_{2} \mathrm{Cl}_{2}\right)$; mp 126 - $129^{\circ} \mathrm{C} ;[\alpha]_{D}{ }^{20}-22.7$ (c 0.2, MeOH); IR $v_{\max }$ (solid) $3330(\mathrm{NH}), 3280(\mathrm{NH}), 3190(\mathrm{OH}), 2950$ $2870(\mathrm{CH}), 1740(\mathrm{C}=\mathrm{O}), 1710(\mathrm{C}=0)$ and $1610(\mathrm{C}=\mathrm{C}) \mathrm{cm}^{-1} ;{ }^{1} \mathrm{H} \mathrm{NMR}\left(600 \mathrm{MHz}, \mathrm{CD}_{3} \mathrm{OD}\right) \delta 8.32(1 \mathrm{H}, \mathrm{s}), 8.19$ $(1 \mathrm{H}, \mathrm{s}), 6.05(1 \mathrm{H}, \mathrm{d}, J=4.5 \mathrm{~Hz}), 5.20(1 \mathrm{H}, \mathrm{t}, J=4.5 \mathrm{~Hz}), 4.76(1 \mathrm{H}, \mathrm{t}, J=5.2 \mathrm{~Hz}), 4.47-4.35(2 \mathrm{H}, \mathrm{m}), 4.32$ $-4.27(1 \mathrm{H}, \mathrm{m}), 3.32-3.29(4 \mathrm{H}, \mathrm{m})$ and $2.63-2.56(28 \mathrm{H}, \mathrm{m}) ;{ }^{13} \mathrm{C} \mathrm{NMR}\left(150 \mathrm{MHz}, \mathrm{CD}_{3} \mathrm{OD}\right) \delta 176.0$, $173.9,157.3,153.9,150.6,141.9,120.6,90.6,83.9,77.7,73.8,64.3,30.1,30.0,17.9$ and 14.6; HRMS calc'd for $\mathrm{C}_{26} \mathrm{H}_{44} \mathrm{~N}_{5} \mathrm{O}_{8} \mathrm{Si}_{2}$ expected 610.2728 , found 610.2730 . 

hydroxy-2,2-dimethyl-4-oxo-4-((3-oxo-3-(propylamino)propyl)amino)butyl) succinate (5). To a solution of acid 32 (480 mg, $0.79 \mathrm{mmol})$ and alcohol 17 (410 mg, $1.58 \mathrm{mmol})$ in $\mathrm{CH}_{2} \mathrm{Cl}_{2}(20 \mathrm{~mL})$ at $0{ }^{\circ} \mathrm{C}$ was added EDC (181 mg, $0.95 \mathrm{mmol})$ and DMAP $(116 \mathrm{mg}, 0.95 \mathrm{mmol})$. The reaction was stirred at RT for $18 \mathrm{~h}$. The reaction was diluted with $\mathrm{CH}_{2} \mathrm{Cl}_{2}(10 \mathrm{~mL})$ and washed with $5 \%$ citric acid solution $(20 \mathrm{~mL})$, sat. $\mathrm{NaHCO}_{3}$ solution $(20 \mathrm{~mL})$, dried $\left(\mathrm{MgSO}_{4}\right)$, filtered and concentrated in vacuo to give crude intermediate 33 (25 mg, 5\%, 70\% purity) as a colourless oil which was used without further purification. The crude oil was dissolved in $\mathrm{MeOH}(7 \mathrm{~mL})$ before ammonium fluoride $(8 \mathrm{mg}, 0.22 \mathrm{mmol})$ was added and the reaction stirred at RT for $90 \mathrm{~min}$. The reaction mixture was concentrated in vacuo. Purification using flash column chromatography (Gradient: 5, 10 then $14 \% \mathrm{MeOH} / \mathrm{CH}_{2} \mathrm{Cl}_{2}$ ) gave 5 (4.9 $\mathrm{mg}, 1 \%$ over two steps) as a colourless oil; $\mathrm{R}_{\mathrm{f}} 0.63\left(15 \% \mathrm{MeOH} / \mathrm{CH}_{2} \mathrm{Cl}_{2}\right) ;[\alpha]_{\mathrm{D}}{ }^{20}-3.8$ (c 0.3, MeOH); IR $v_{\max }$ (oil) $3320(\mathrm{NH}), 3250(\mathrm{NH}), 3150(\mathrm{OH}), 2960-2880(\mathrm{CH}), 1730(\mathrm{C}=\mathrm{O}), 1640(\mathrm{C}=\mathrm{O})$ and $1570(\mathrm{C}=\mathrm{C})$ $\mathrm{cm}^{-1} ;{ }^{1} \mathrm{H}$ NMR $\left(600 \mathrm{MHz}, \mathrm{CD}_{3} \mathrm{OD}\right) \delta 8.29(1 \mathrm{H}, \mathrm{s}), 8.21(1 \mathrm{H}, \mathrm{s}), 6.01(1 \mathrm{H}, \mathrm{d}, J=4.6 \mathrm{~Hz}), 4.74(1 \mathrm{H}, \mathrm{t}, J=4.6$ $\mathrm{Hz}), 4.44-4.35(3 \mathrm{H}, \mathrm{m}), 4.27-4.24(1 \mathrm{H}, \mathrm{m}), 4.03(1 \mathrm{H}, \mathrm{d}, J=10.5), 3.89(1 \mathrm{H}, \mathrm{d}, J=10.5 \mathrm{~Hz}), 3.86(1 \mathrm{H}$, s), $3.51-3.39(2 \mathrm{H}, \mathrm{m}), 3.13-3.08(2 \mathrm{H}, \mathrm{m}), 2.66-2.64(4 \mathrm{H}, \mathrm{m}), 2.43-2.38(2 \mathrm{H}, \mathrm{m}), 1.53-1.45(2 \mathrm{H}$, m), $0.96(3 \mathrm{H}, \mathrm{s})$ and $0.93-0.89(6 \mathrm{H}, \mathrm{m}) ;{ }^{13} \mathrm{C} \mathrm{NMR}\left(150 \mathrm{MHz}, \mathrm{CD}_{3} \mathrm{OD}\right) \delta 175.4,173.9,173.8,173.6$, $157.3,153.9,150.6,141.3,120.5,90.3,83.5,75.8,75.2$, 71.7, 71.5, 65.1, 42.3, 39.3, 36.4, 30.3, 23.5, 21.6, 20.9 and 11.7; HRMS calc'd for $\mathrm{C}_{26} \mathrm{H}_{40} \mathrm{~N}_{7} \mathrm{O}_{10}$ expected 610.2837, found 610.2830.

((5aS,6S,8S,8aS)-8-(6-Amino-9H-purin-9-yl)-2,2,4,4-tetraisopropyltetrahydrofuro[3,4-

$f][1,3,5,2,4]$ trioxadisilepin-6-yl)methyl benzyl glutarate (35). To a solution of alcohol $26(1.00 \mathrm{~g}, 1.96$ $\mathrm{mmol}$ ) and protected glutaric acid $34(870 \mathrm{mg}, 3.92 \mathrm{mmol})$ in $\mathrm{CH}_{2} \mathrm{Cl}_{2}(15 \mathrm{~mL})$ was added DCC $(510 \mathrm{mg}$, $2.55 \mathrm{mmol}$ ) and DMAP (60 mg, $0.49 \mathrm{mmol}$ ). The reaction was stirred at RT for $16 \mathrm{~h}$. The insoluble dicyclohexylurea was removed by filtration, washed with $\mathrm{CH}_{2} \mathrm{Cl}_{2}$. The organic filtrate was washed with sat. $\mathrm{NaHCO}_{3}$ solution $(3 \times 20 \mathrm{~mL})$, brine $(20 \mathrm{~mL})$, dried $\left(\mathrm{MgSO}_{4}\right)$, filtered and concentrated in vacuo. Purification using flash column chromatography (Gradient: $0-2 \% \mathrm{MeOH} / \mathrm{CH}_{2} \mathrm{Cl}_{2}$ ) gave $35(480 \mathrm{mg}$, 34\%) as an off-white solid; $\mathrm{R}_{\mathrm{f}} 0.54\left(5 \% \mathrm{MeOH} / \mathrm{CH}_{2} \mathrm{Cl}_{2}\right)$; mp 111 - $113{ }^{\circ} \mathrm{C} ;[\alpha]_{\mathrm{D}}{ }^{20}-24.2$ (c $\left.0.1, \mathrm{MeOH}\right) ; \mathrm{IR}$ $V_{\max }$ (solid) $3310(\mathrm{NH}), 3180(\mathrm{NH}), 2940-2870(\mathrm{CH}), 1740$ (C=O) and $1600(\mathrm{C}=\mathrm{C}) \mathrm{cm}^{-1} ;{ }^{1} \mathrm{H}$ NMR (600 $\left.\mathrm{MHz}, \mathrm{CDCl}_{3}\right) \delta 8.30(1 \mathrm{H}, \mathrm{s}), 7.97(1 \mathrm{H}, \mathrm{s}), 7.39-7.29(5 \mathrm{H}, \mathrm{m}), 6.01-5.97(3 \mathrm{H}, \mathrm{m}), 5.11(2 \mathrm{H}, \mathrm{s}), 5.09(1 \mathrm{H}$, $\mathrm{dd}, J=4.7$ and $3.3 \mathrm{~Hz}), 4.69(1 \mathrm{H}, \mathrm{dd}, J=6.4$ and $4.7 \mathrm{~Hz}), 4.48-4.44(1 \mathrm{H}, \mathrm{m}), 4.34-4.29(2 \mathrm{H}, \mathrm{m}), 2.44$ $-2.38(4 \mathrm{H}, \mathrm{m}), 1.96(2 \mathrm{H}, \mathrm{q}, J=7.4 \mathrm{~Hz})$ and $1.10-1.00(28 \mathrm{H}, \mathrm{m}) ;{ }^{13} \mathrm{C} \mathrm{NMR}\left(150 \mathrm{MHz}, \mathrm{CDCl}_{3}\right) \delta 172.7$, 155.6, 152.9, 149.6, 139.8, 136.0, 128.7, 128.6, 128.5, 120.4, 90.5, 81.8, 75.7, 72.7, 66.4, 63.7, 33.6, 33.5, 20.2, 17.3 and 13.4; HRMS calc'd for $\mathrm{C}_{34} \mathrm{H}_{52} \mathrm{O}_{8} \mathrm{~N}_{5} \mathrm{Si}_{2}$ expected 714.3354, found 714.3351.

5-(((5aS,6S,8S,8aS)-8-(6-Amino-9H-purin-9-yl)-2,2,4,4-tetraisopropyltetrahydrofuro[3,4$f][1,3,5,2,4]$ trioxadisilepin-6-yl)methoxy)-5-oxopentanoic acid (36). To protected adenosine 
analogue 35 (450 mg, $0.63 \mathrm{mmol})$ and $\mathrm{Pd} / \mathrm{C}(10 \mathrm{wt} \%, 17 \mathrm{mg}, 0.16 \mathrm{mmol})$ was added $\mathrm{MeOH}(10 \mathrm{~mL})$ before evacuating and filling the flask with hydrogen. The reaction was stirred at RT under a hydrogen atmosphere for $3 \mathrm{~h}$. The reaction mixture was filtered through Celite ${ }^{\circ}$, washed with $\mathrm{MeOH}$ and the filtrate concentrated in vacuo to give $36(387 \mathrm{mg}, 97 \%)$ as an off-white solid; $R_{f} 0.31$ (10\% $\mathrm{MeOH} / \mathrm{CH}_{2} \mathrm{Cl}_{2}$ ); mp $137-138^{\circ} \mathrm{C}$; $[\alpha]_{\mathrm{D}}{ }^{20}-17.6$ (c 0.2, MeOH); IR $v_{\max }$ (solid) 3320 (NH), 3280 (NH), 3150 (OH), $2950-2860(\mathrm{CH}), 1740(\mathrm{C}=\mathrm{O}), 1700(\mathrm{C}=\mathrm{O})$ and $1610(\mathrm{C}=\mathrm{C}) \mathrm{cm}^{-1} ;{ }^{1} \mathrm{H}$ NMR (600 MHz, CD $\left.{ }_{3} \mathrm{OD}\right) \delta$ $8.29(1 \mathrm{H}, \mathrm{s}), 8.19(1 \mathrm{H}, \mathrm{s}), 6.04(1 \mathrm{H}, \mathrm{d}, J=4.4 \mathrm{~Hz}), 5.26(1 \mathrm{H}, \mathrm{t}, J=4.7 \mathrm{~Hz}), 4.78(1 \mathrm{H}, \mathrm{t}, J=5.1 \mathrm{~Hz}), 4.45-$ $4.32(2 \mathrm{H}, \mathrm{m}), 4.29(1 \mathrm{H}, \mathrm{q}, J=4.4 \mathrm{~Hz}), 2.40-2.28(4 \mathrm{H}, \mathrm{m}), 1.89-1.81(2 \mathrm{H}, \mathrm{m})$ and $1.17-0.89(28 \mathrm{H}, \mathrm{m})$; ${ }^{13} \mathrm{C}$ NMR $\left(150 \mathrm{MHz}, \mathrm{CD}_{3} \mathrm{OD}\right) \delta$ 177.0, 174.2, 157.4, 153.9, 150.6, 141.6, 120.6, 90.7, 83.7, 76.8, 74.0, 64.1, 34.1, 34.0, 21.4, 17.6 and 14.7; HRMS calc'd for $\mathrm{C}_{28} \mathrm{H}_{42} \mathrm{~N}_{5} \mathrm{O}_{8} \mathrm{Si}_{2}$ expected 624.2881, found 624.2908 .

((2S,3R,4S,5S)-5-(6-Amino-9H-purin-9-yl)-3,4-dihydroxytetrahydrofuran-2-yl)methyl

$((R)-3-$ hydroxy-2,2-dimethyl-4-oxo-4-((3-oxo-3-(propylamino)propyl)amino)butyl) glutarate (6). To a solution of acid 36 (350 mg, $0.56 \mathrm{mmol})$ and alcohol 17 (175 mg, $0.67 \mathrm{mmol})$ in $\mathrm{CH}_{2} \mathrm{Cl}_{2}(17 \mathrm{~mL})$ at $0{ }^{\circ} \mathrm{C}$ was added EDC (128 mg, $0.67 \mathrm{mmol}$ ) and DMAP ( $82 \mathrm{mg}, 0.67 \mathrm{mmol})$. The reaction was stirred at RT for $18 \mathrm{~h}$. The reaction was diluted with $\mathrm{CH}_{2} \mathrm{Cl}_{2}(10 \mathrm{~mL})$ and washed with $5 \%$ citric acid solution $(20 \mathrm{~mL})$, sat. $\mathrm{NaHCO}_{3}$ solution $(20 \mathrm{~mL})$, dried $\left(\mathrm{MgSO}_{4}\right)$, filtered and concentrated in vacuo to give crude intermediate 37 (65 mg, 13\%, 60\% purity) as a colourless oil which was used without further purification. The crude oil was dissolved in $\mathrm{MeOH}(20 \mathrm{~mL})$ before ammonium fluoride $(23 \mathrm{mg}$, $0.64 \mathrm{mmol}$ ) was added and the reaction stirred at RT for $90 \mathrm{~min}$. The reaction mixture was concentrated in vacuo. Purification using flash column chromatography (Gradient: 5, 10 then 14\% $\mathrm{MeOH} / \mathrm{CH}_{2} \mathrm{Cl}_{2}$ ) gave 6 (17 mg, 3\% over two steps) as a colourless gum; $\mathrm{R}_{\mathrm{f}} 0.31$ (15\% $\mathrm{MeOH} / \mathrm{CH}_{2} \mathrm{Cl}_{2}$ ); $[\alpha]_{D}^{20}-0.7$ (c 0.8, MeOH); IR $v_{\max }$ (solid) $3340(\mathrm{NH}), 3260(\mathrm{NH}), 3090(\mathrm{OH}), 2970-2880(\mathrm{CH}), 1720$ (C=O), $1650(\mathrm{C}=0)$ and $1590(\mathrm{C}=\mathrm{C}) \mathrm{cm}^{-1} ;{ }^{1} \mathrm{H}$ NMR $\left(600 \mathrm{MHz}, \mathrm{CD}_{3} \mathrm{OD}\right) \delta 8.27(1 \mathrm{H}, \mathrm{s}), 8.20(1 \mathrm{H}, \mathrm{s}), 6.01(1 \mathrm{H}, \mathrm{d}, J$ $=4.4 \mathrm{~Hz}), 4.75(1 \mathrm{H}, \mathrm{t}, J=4.8 \mathrm{~Hz}), 4.43-4.35(3 \mathrm{H}, \mathrm{m}), 4.27-4.23(1 \mathrm{H}, \mathrm{m}), 4.02(1 \mathrm{H}, \mathrm{d}, J=9.5 \mathrm{~Hz}), 3.90$ $(1 \mathrm{H}, \mathrm{d}, J=9.5 \mathrm{~Hz}), 3.86(1 \mathrm{H}, \mathrm{s}), 3.51-3.39(2 \mathrm{H}, \mathrm{m}), 3.12-3.08(2 \mathrm{H}, \mathrm{m}), 2.50-2.35(6 \mathrm{H}, \mathrm{m}), 1.92-1.85$ $(2 \mathrm{H}, \mathrm{m}), 1.53-1.45(2 \mathrm{H}, \mathrm{m}), 0.96(3 \mathrm{H}, \mathrm{s})$ and $0.94-0.88(6 \mathrm{H}, \mathrm{m}) ;{ }^{13} \mathrm{C} \mathrm{NMR}\left(150 \mathrm{MHz}, \mathrm{CD}_{3} \mathrm{OD}\right) \delta 175.4$, 174.6, 173.9, 173.5, 157.3, 153.9, 150.6, 141.2, 120.6, 90.4, 83.4, 75.8, 75.1, 71.8, 71.3, 64.8, 42.3, 39.4, 36.8, 33.8, 23.6, 21.6, 21.1, 20.8 and 11.8; $\mathrm{HRMS}$ calc'd for $\mathrm{C}_{27} \mathrm{H}_{42} \mathrm{~N}_{7} \mathrm{O}_{10}$ expected 624.2993, found 624.3005 .

((5aS,6S,8S,8aS)-8-(6-Amino-9H-purin-9-yl)-2,2,4,4-tetraisopropyltetrahydrofuro[3,4$f][1,3,5,2,4]$ trioxadisilepin-6-yl)methyl neopentyl succinate (39). To a solution of acid 32 (330 mg, $0.54 \mathrm{mmol})$ in $\mathrm{CH}_{2} \mathrm{Cl}_{2}(10 \mathrm{~mL})$ at $0^{\circ} \mathrm{C}$ was added DIC (125 $\left.\mu \mathrm{L}, 0.81 \mathrm{mmol}\right)$ and DMAP (17 mg, $\left.0.14 \mathrm{mmol}\right)$. The reaction was stirred at $0{ }^{\circ} \mathrm{C}$ for $10 \mathrm{~min}$ before 2,2-dimethyl-1-propanol $(71 \mathrm{mg}, 0.81 \mathrm{mmol})$ in 
$\mathrm{CH}_{2} \mathrm{Cl}_{2}(5 \mathrm{~mL})$ was added dropwise. The reaction was stirred at $\mathrm{RT}$ for $20 \mathrm{~h}$. The insoluble material was filtered and washed with $\mathrm{CH}_{2} \mathrm{Cl}_{2}$. The organic filtrate was washed with $5 \%$ citric acid solution $(30 \mathrm{~mL})$, then sat. $\mathrm{NaHCO}_{3}$ solution $(30 \mathrm{~mL})$, dried $\left(\mathrm{MgSO}_{4}\right)$, filtered and concentrated in vacuo. Purification using flash column chromatography (Gradient: $1-4 \% \mathrm{MeOH} / \mathrm{CH}_{2} \mathrm{Cl}_{2}$ ) gave $39(243 \mathrm{mg}, 66 \%$ ) as an offwhite solid; $\mathrm{R}_{\mathrm{f}} 0.31$ ( $8 \% \mathrm{MeOH} / \mathrm{CH}_{2} \mathrm{Cl}_{2}$ ); $\mathrm{mp} 113-115^{\circ} \mathrm{C}$; $[\alpha]_{\mathrm{D}}{ }^{20}-17.5$ (c 0.2, MeOH); IR v $\max$ (solid) 3340 $(\mathrm{NH}), 3180(\mathrm{NH}), 2950-2870(\mathrm{CH}), 1740(\mathrm{C}=\mathrm{O})$ and $1610(\mathrm{C}=\mathrm{C}) \mathrm{cm}^{-1} ;{ }^{1} \mathrm{H} \mathrm{NMR}\left(600 \mathrm{MHz}, \mathrm{CDCl}_{3}\right) \delta 8.31$ $(1 \mathrm{H}, \mathrm{s}), 8.00(1 \mathrm{H}, \mathrm{s}), 5.99(1 \mathrm{H}, \mathrm{d}, J=3.3 \mathrm{~Hz}), 5.90(2 \mathrm{H}, \mathrm{br}), 5.08(1 \mathrm{H}, \mathrm{dd}, J=4.7$ and $3.3 \mathrm{~Hz}), 4.69(1 \mathrm{H}, \mathrm{t}$, $J=4.7 \mathrm{~Hz}), 4.50-4.46(1 \mathrm{H}, \mathrm{m}), 4.35-4.31(2 \mathrm{H}, \mathrm{m}), 3.77(2 \mathrm{H}$, apparent s), $2.67-2.65(4 \mathrm{H}, \mathrm{m}), 1.09-$ $1.01(28 \mathrm{H}, \mathrm{m})$ and $0.91(9 \mathrm{H}, \mathrm{s}) ;{ }^{13} \mathrm{C} \mathrm{NMR}\left(150 \mathrm{MHz} \mathrm{CDCl}_{3}\right) \delta 172.3,155.4,152.7,149.6,139.7,120.5$, 90.4, 81.8, 75.8, 74.3, 72.7, 63.9, 31.4, 29.1, 26.5, 17.3 and 13.2; HRMS calc'd for $\mathrm{C}_{31} \mathrm{H}_{54} \mathrm{O}_{8} \mathrm{~N}_{5} \mathrm{Si}_{2}$ expected 679.3427, found 679.34269 .

((2S,3R,4S,5S)-5-(6-Amino-9H-purin-9-yl)-3,4-dihydroxytetrahydrofuran-2-yl)methyl

neopentyl succinate (7). To a solution of protected CoA analogue 39 (80.0 mg, $0.12 \mathrm{mmol}$ ) in $\mathrm{MeOH}(3 \mathrm{~mL})$ was added ammonium fluoride $(37 \mathrm{mg}, 1.02 \mathrm{mmol})$. The reaction was stirred at RT for $1.5 \mathrm{~h}$. The reaction was concentrated in vacuo. Purification using flash column chromatography (Gradient: 3, 5 then 7\% $\mathrm{MeOH} / \mathrm{CH}_{2} \mathrm{Cl}_{2}$ ) gave 7 (31 mg, 59\%) as a colourless oil; $\mathrm{R}_{\mathrm{f}} 0.45\left(10 \% \mathrm{MeOH} / \mathrm{CH}_{2} \mathrm{Cl}_{2}\right) ; \mathrm{mp} 75-77^{\circ} \mathrm{C}$; $[\alpha]_{D}^{20}-5.8$ (c 0.4, MeOH); IR v $\max$ (oil) $3330(\mathrm{NH}), 3270(\mathrm{NH}), 3130(\mathrm{OH}), 2960-2910(\mathrm{CH}), 1730(\mathrm{C}=\mathrm{O})$, $1650(\mathrm{C}=\mathrm{O})$ and $1610(\mathrm{C}=\mathrm{C}) \mathrm{cm}^{-1}{ }^{1}{ }^{1} \mathrm{H}$ NMR $\left(600 \mathrm{MHz}, \mathrm{CD}_{3} \mathrm{OD}\right) \delta 8.28(1 \mathrm{H}, \mathrm{s}), 8.20(1 \mathrm{H}, \mathrm{s}), 6.01(1 \mathrm{H}, \mathrm{d}, J$ $=4.5 \mathrm{~Hz}), 4.74(1 \mathrm{H}, \mathrm{t}, J=4.9 \mathrm{~Hz}), 4.44-4.33(3 \mathrm{H}, \mathrm{m}), 4.27-4.23(1 \mathrm{H}, \mathrm{m}), 3.76(1 \mathrm{H}, \mathrm{d}, J=10.5 \mathrm{~Hz}), 3.73$ $(1 \mathrm{H}, \mathrm{d}, J=10.5 \mathrm{~Hz}), 3.32-3.29(4 \mathrm{H}, \mathrm{m})$ and $2.65(9 \mathrm{H}, \mathrm{s}) ;{ }^{13} \mathrm{C} \mathrm{NMR}\left(150 \mathrm{MHz}, \mathrm{CD}_{3} \mathrm{OD}\right) \delta 174.0,173.8$, 157.3, 153.9, 150.6, 141.3, 120.5, 90.3, 83.5, 75.1, 75.0, 71.8, 65.1, 32.1, 29.9 and 26.7; HRMS calc'd for $\mathrm{C}_{19} \mathrm{H}_{28} \mathrm{~N}_{8} \mathrm{O}_{7}$ expected 438.1983 , found 438.1982 .

\section{((5aS,6S,8S,8aS)-8-(6-Amino-9H-purin-9-yl)-2,2,4,4-tetraisopropyltetrahydrofuro[3,4-}

$f][1,3,5,2,4]$ trioxadisilepin-6-yl)methyl $\quad((R)$-3-hydroxy-2,2-dimethyl-4-(methylamino)-4-oxobutyl) succinate (41). To a solution of acid $32(310 \mathrm{mg}, 0.51 \mathrm{mmol})$ in $\mathrm{CH}_{2} \mathrm{Cl}_{2}(10 \mathrm{~mL})$ at $0{ }^{\circ} \mathrm{C}$ was added DIC $(120 \mu \mathrm{L}, 0.77 \mathrm{mmol})$ and DMAP $(16 \mathrm{mg}, 0.13 \mathrm{mmol})$. The reaction was stirred at $0{ }^{\circ} \mathrm{C}$ for $10 \mathrm{~min}$ before alcohol 40 (123 mg, $0.77 \mathrm{mmol}$ ) in $\mathrm{CH}_{2} \mathrm{Cl}_{2}(5 \mathrm{~mL}$ ) was added dropwise. The reaction was stirred at $\mathrm{RT}$ for $20 \mathrm{~h}$. The insoluble material was filtered and washed with $\mathrm{CH}_{2} \mathrm{Cl}_{2}$. The organic filtrate was washed with $5 \%$ citric acid solution $(30 \mathrm{~mL})$, then sat. $\mathrm{NaHCO}_{3}$ solution $(30 \mathrm{~mL})$, dried $\left(\mathrm{MgSO}_{4}\right)$, filtered and concentrated in vacuo. Purification using flash column chromatography (Gradient: 1 - 5\% $\mathrm{MeOH} / \mathrm{CH}_{2} \mathrm{Cl}_{2}$ ) gave 41 (175 mg, 46\%) as an off-white solid; $\mathrm{R}_{\mathrm{f}} 0.51\left(10 \% \mathrm{MeOH} / \mathrm{CH}_{2} \mathrm{Cl}_{2}\right) ; \mathrm{mp} 109-111$ ${ }^{\circ} \mathrm{C} ;[\alpha]_{D}{ }^{20}-8.8$ (c 0.1, MeOH); IR $v_{\max }$ (solid) $3330(\mathrm{NH}), 3290(\mathrm{NH}), 3180(\mathrm{OH}), 2940-2870(\mathrm{CH}), 1730$ $(\mathrm{C}=0), 1640(\mathrm{C}=0)$ and $1600(\mathrm{C}=\mathrm{C}) \mathrm{cm}^{-1} ;{ }^{1} \mathrm{H}$ NMR $\left(600 \mathrm{MHz}, \mathrm{CDCl}_{3}\right) \delta 8.29(1 \mathrm{H}, \mathrm{s}), 8.09(1 \mathrm{H}, \mathrm{s}), 6.81(1 \mathrm{H}$, $\mathrm{d}, J=5.0 \mathrm{~Hz}), 6.13(2 \mathrm{H}, \mathrm{br}), 6.02(1 \mathrm{H}, \mathrm{d}, J=2.6 \mathrm{~Hz}), 5.06(1 \mathrm{H}, \mathrm{dd}, J=4.7$ and $2.6 \mathrm{~Hz}), 4.70(1 \mathrm{H}, \mathrm{dd}, J=$ 
6.8 and $4.7 \mathrm{~Hz}), 4.56-4.50(1 \mathrm{H}, \mathrm{m}), 4.36-4.28(2 \mathrm{H}, \mathrm{m}), 4.23(1 \mathrm{H}, \mathrm{d}, J=10.8 \mathrm{~Hz}), 4.05(1 \mathrm{H}, \mathrm{s}), 3.84$ $(1 \mathrm{H}, \mathrm{d}, J=10.8 \mathrm{~Hz}), 2.83(3 \mathrm{H}, \mathrm{d}, J=5.0 \mathrm{~Hz}), 2.63-2.58(4 \mathrm{H}, \mathrm{m}), 1.10-1.04(31 \mathrm{H}, \mathrm{m})$ and $0.93(3 \mathrm{H}, \mathrm{s})$; ${ }^{13} \mathrm{C}$ NMR $\left(150 \mathrm{MHz}, \mathrm{CDCl}_{3}\right) \delta 173.2,172.7,172.2,155.2,152.6,149.4,139.8,120.0,90.6,81.7,76.2$, 74.4, 72.2, 71.0, 63.0, 38.7, 29.5, 25.8, 21.5, 19.9, 17.5 and 13.6; HRMS calc'd for $\mathrm{C}_{33} \mathrm{H}_{57} \mathrm{O}_{10} \mathrm{~N}_{6} \mathrm{Si}_{2}$ expected 753.3675 , found 753.3679 .

((2S,3R,4S,5S)-5-(6-Amino-9H-purin-9-yl)-3,4-dihydroxytetrahydrofuran-2-yl)methyl

$((R)-3-$ hydroxy-2,2-dimethyl-4-(methylamino)-4-oxobutyl) succinate (8). To a solution of protected CoA analogue 41 (60.0 mg, $0.08 \mathrm{mmol}$ ) in $\mathrm{MeOH}(2 \mathrm{~mL}$ ) was added ammonium fluoride ( $25 \mathrm{mg}, 0.68 \mathrm{mmol}$ ) and the reaction was stirred at RT for $1.5 \mathrm{~h}$. The reaction mixture was concentrated in vacuo. Purification using flash column chromatography (Gradient: $5 \%$ then $10 \% \mathrm{MeOH} / \mathrm{CH}_{2} \mathrm{Cl}_{2}$ ) gave $8(14 \mathrm{mg}$, $34 \%$ ) as a colourless oil; $\mathrm{R}_{\mathrm{f}} 0.17\left(10 \% \mathrm{MeOH} / \mathrm{CH}_{2} \mathrm{Cl}_{2}\right) ; \mathrm{mp} 86-88^{\circ} \mathrm{C}$; $[\alpha]_{\mathrm{D}}{ }^{20}-1.8$ (c $\left.0.9, \mathrm{MeOH}\right) ; \mathrm{IR} v_{\max }$ (solution in $\left.\mathrm{CH}_{2} \mathrm{Cl}_{2}\right) 3360(\mathrm{NH}), 3240(\mathrm{NH}), 3090(\mathrm{OH}), 2980-2860(\mathrm{CH}), 1740(\mathrm{C}=\mathrm{O}), 1650(\mathrm{C}=\mathrm{O})$ and $1600(\mathrm{C}=\mathrm{C}) \mathrm{cm}^{-1} ;{ }^{1} \mathrm{H}$ NMR $\left(600 \mathrm{MHz}, \mathrm{CD}_{3} \mathrm{OD}\right) \delta 8.29(1 \mathrm{H}, \mathrm{s}), 8.20(1 \mathrm{H}, \mathrm{s}), 6.02(1 \mathrm{H}, \mathrm{d}, J=4.5 \mathrm{~Hz}), 4.74$ $(1 \mathrm{H}, \mathrm{t}, J=4.9 \mathrm{~Hz}), 4.55-4.35(3 \mathrm{H}, \mathrm{m}), 4.27-4.23(1 \mathrm{H}, \mathrm{m}), 4.03(1 \mathrm{H}, \mathrm{d}, J=10.6 \mathrm{~Hz}), 3.90(1 \mathrm{H}, \mathrm{d}, J=10.6$ $\mathrm{Hz}), 3.86(1 \mathrm{H}, \mathrm{s}), 2.74(3 \mathrm{H}, \mathrm{s}), 2.66-2.63(4 \mathrm{H}, \mathrm{m}), 0.96(3 \mathrm{H}, \mathrm{s})$ and $0.92(3 \mathrm{H}, \mathrm{s}) ;{ }^{13} \mathrm{C} \mathrm{NMR}(150 \mathrm{MHz}$, $\left.\mathrm{CD}_{3} \mathrm{OD}\right) \delta$ 176.1, 173.9, 173.8, 157.3, 153.9, 150.6, 141.2, 120.5, 90.3, 83.5, 76.0, 75.2, 71.8, 71.4, 65.1, 39.3, 29.9, 25.8, 21.5 and 20.8; HRMS calc'd for $\mathrm{C}_{27} \mathrm{H}_{31} \mathrm{~N}_{6} \mathrm{O}_{9}$ expected 511.2147, found 511.2146.

(R)-2,4-Dihydroxy-3,3-dimethyl-N-(3-(methylamino)-3-oxopropyl)butanamide (42). To Boc protected $N$-methyl-6-aminopropionamide $(2.11 \mathrm{~g}, 10.4 \mathrm{mmol})$ in $\mathrm{CH}_{2} \mathrm{Cl}_{2}(80 \mathrm{~mL})$ was added TFA (20 $\mathrm{mL}$ ). The reaction was stirred at RT for $4 \mathrm{~h}$. The reaction was concentrated in vacuo to give $N$-methyl6-aminopropionamide ( $3.50 \mathrm{~g}$, quantitative) as the TFA salt which was used without any further purification. The crude oil was dissolved in $\mathrm{MeOH}(60 \mathrm{~mL})$ to which was added $\mathrm{NEt}_{3}(12 \mathrm{~mL})$. The solution was heated at reflux for $90 \mathrm{~min}$ before D-pantolactone $(1.40 \mathrm{~g}, 10.8 \mathrm{mmol})$ was added. The reaction was heated at reflux for $20 \mathrm{~h}$. The reaction mixture was concentrated in vacuo. Purification using flash column chromatography (Gradient: $0,2,4$ and $6 \% \mathrm{MeOH} / \mathrm{CH}_{2} \mathrm{Cl}_{2}$ ) gave the $\mathrm{NEt}_{3}$ salt of 42 (835 mg, 34\% over two steps) as a colourless oil; $\mathrm{R}_{\mathrm{f}} 0.36\left(10 \% \mathrm{MeOH} / \mathrm{CH}_{2} \mathrm{Cl}_{2}\right)$; $[\alpha]_{D}{ }^{20}+12.0$ (c 0.3 , $\mathrm{MeOH}$ ); IR $v_{\max }$ (oil) $3300(\mathrm{NH}), 3250(\mathrm{NH}), 3080(\mathrm{OH}), 2950-2870(\mathrm{CH}), 1740(\mathrm{C}=\mathrm{O})$ and $1640(\mathrm{C}=\mathrm{O})$ $\mathrm{cm}^{-1} ;{ }^{1} \mathrm{H}$ NMR $\left(600 \mathrm{MHz}, \mathrm{CD}_{3} \mathrm{OD}\right) \delta 3.89(1 \mathrm{H}, \mathrm{s}), 3.54-3.41(3 \mathrm{H}, \mathrm{m}), 3.38(1 \mathrm{H}, \mathrm{d}, J=11.0 \mathrm{~Hz}), 2.71(3 \mathrm{H}$, s), $2.41(2 \mathrm{H}, \mathrm{t}, J=6.7 \mathrm{~Hz}), 0.91(3 \mathrm{H}, \mathrm{s})$ and $0.90(3 \mathrm{H}, \mathrm{s}) ;{ }^{13} \mathrm{C} \mathrm{NMR}\left(150 \mathrm{MHz}, \mathrm{CD}_{3} \mathrm{OD}\right) \delta 176.1,174.3,77.3$, 70.3, 40.3, 36.4, 26.5, 21.3 and 20.9; HRMS calc'd for $\mathrm{C}_{10} \mathrm{H}_{21} \mathrm{~N}_{2} \mathrm{O}_{4}$ expected 233.1496, found 233.1495. solution of acid $32(300 \mathrm{mg}, 0.43 \mathrm{mmol})$ in $\mathrm{CH}_{2} \mathrm{Cl}_{2}(9 \mathrm{~mL})$ at $0{ }^{\circ} \mathrm{C}$ was added DIC (100 $\left.\mu \mathrm{L}, 0.65 \mathrm{mmol}\right)$ 
and DMAP (13 mg, $0.11 \mathrm{mmol})$. The reaction was stirred at $0{ }^{\circ} \mathrm{C}$ for $10 \mathrm{~min}$ before alcohol $42(200 \mathrm{mg}$, $0.86 \mathrm{mmol})$ in $\mathrm{CH}_{2} \mathrm{Cl}_{2}(5 \mathrm{~mL})$ was added dropwise. The reaction was stirred at $\mathrm{RT}$ for $20 \mathrm{~h}$. The resulting precipitate was filtered and washed with $\mathrm{CH}_{2} \mathrm{Cl}_{2}$. The organic filtrate was washed with $5 \%$ citric acid solution $(20 \mathrm{~mL})$, then sat. $\mathrm{NaHCO}_{3}$ solution $(20 \mathrm{~mL})$, dried $\left(\mathrm{MgSO}_{4}\right)$, filtered and concentrated in vacuo. Purification using flash column chromatography (Gradient: $1-10 \% \mathrm{MeOH} / \mathrm{CH}_{2} \mathrm{Cl}_{2}$ ) gave the crude intermediate ( $182 \mathrm{mg}, 46 \%, 75 \%$ purity) as a colourless oil which was used without further purification. The crude oil ( $85 \mathrm{mg}$ ) was dissolved in $\mathrm{MeOH}(20 \mathrm{~mL}$ ) before ammonium fluoride ( $31 \mathrm{mg}, 0.85 \mathrm{mmol}$ ) was added and the reaction stirred at RT for $1 \mathrm{~h}$. The reaction mixture was concentrated in vacuo. Purification using flash column chromatography (Gradient: 10, 12 then $15 \% \mathrm{MeOH} / \mathrm{CH}_{2} \mathrm{Cl}_{2}$ ) gave 9 (22 $\mathrm{mg}, 18 \%$ over two steps) as a colourless gum; $\mathrm{R}_{\mathrm{f}} 0.36\left(15 \% \mathrm{MeOH} / \mathrm{CH}_{2} \mathrm{Cl}_{2}\right) ;[\alpha]_{\mathrm{D}}{ }^{20}-1.5$ (c 0.9, MeOH); IR $v_{\max }$ (solid) $3340(\mathrm{NH}), 3290(\mathrm{NH}), 3100(\mathrm{OH}), 2980-2920(\mathrm{CH}), 1730(\mathrm{C}=\mathrm{O}), 1690(\mathrm{C}=\mathrm{O})$ and 1550 $(\mathrm{C}=\mathrm{C}) \mathrm{cm}^{-1} ;{ }^{1} \mathrm{H}$ NMR $\left(600 \mathrm{MHz}, \mathrm{CD}_{3} \mathrm{OD}\right) \delta 8.50(1 \mathrm{H}, \mathrm{s}), 8.41(1 \mathrm{H}, \mathrm{s}), 6.08(1 \mathrm{H}, \mathrm{d}, J=4.5 \mathrm{~Hz}), 4.74(1 \mathrm{H}, \mathrm{t}, J$ $=4.8 \mathrm{~Hz}), 4.42-4.34(3 \mathrm{H}, \mathrm{m}), 4.30-4.27(1 \mathrm{H}, \mathrm{m}), 4.02(1 \mathrm{H}, \mathrm{d}, J=10.6 \mathrm{~Hz}), 3.88(1 \mathrm{H}, \mathrm{d}, J=10.6 \mathrm{~Hz})$, $3.84(1 \mathrm{H}, \mathrm{s}), 3.52-3.39(2 \mathrm{H}, \mathrm{m}), 2.70-2.68(4 \mathrm{H}, \mathrm{m}), 2.65(3 \mathrm{H}, \mathrm{s}), 2.41(2 \mathrm{H}, \mathrm{t}, J=6.6 \mathrm{~Hz}), 0.95(3 \mathrm{H}, \mathrm{s})$ and $0.92(3 \mathrm{H}, \mathrm{s}) ;{ }^{13} \mathrm{C}$ NMR $\left(150 \mathrm{MHz}, \mathrm{CD}_{3} \mathrm{OD}\right) \delta 175.4,174.0,173.9,173.8,152.3,150.0,145.9,143.9$, $120.7,90.7,83.9,75.9,75.5,71.7,71.5,64.9$ 39.5, 36.4, 29.9, 26.4, 21.5 and 20.8; HRMS calc'd for $\mathrm{C}_{24} \mathrm{H}_{36} \mathrm{~N}_{7} \mathrm{O}_{10}$ expected 582.2518, found 582.2516.

$(10 R, 29 R)-1-((2 R, 3 S, 4 R, 5 R)-5-(6-A m i n o-9 H-p u r i n-9-y l)-3,4-d i h y d r o x y t e t r a h y d r o f u r a n-2-y l)-10,29-$ dihydroxy-9,9,30,30-tetramethyl-3,6,11,15,24,28-hexaoxo-2,7-dioxa-19,20-dithia-12,16,23,27tetraazahentriacontan-31-yl (( $(2 S, 3 R, 4 S, 5 S)-5-(6-$ amino-9H-purin-9-yl)-3,4dihydroxytetrahydrofuran-2-yl)methyl) succinate (46). To a solution of acid 32 (700 mg, $1.15 \mathrm{mmol}$ ) in THF (50 mL) at $0^{\circ} \mathrm{C}$ was added DIC ( $\left.180 \mu \mathrm{L}, 1.15 \mathrm{mmol}\right)$ and DMAP $(56 \mathrm{mg}, 0.46 \mathrm{mmol})$. The reaction was stirred at $0{ }^{\circ} \mathrm{C}$ for $10 \mathrm{~min}$ before D-pantethine 43 ( $255 \mathrm{mg}, 0.46 \mathrm{mmol}$ ) in THF ( $25 \mathrm{~mL}$ ) was added dropwise. The reaction was stirred at RT for $20 \mathrm{~h}$. The reaction was concentrated in vacuo. The resulting residue was dissolved in $\mathrm{CH}_{2} \mathrm{Cl}_{2}(40 \mathrm{~mL}$ ) and washed with $5 \%$ citric acid solution (40 mL), sat. $\mathrm{NaHCO}_{3}$ solution $(40 \mathrm{~mL})$, dried $\left(\mathrm{MgSO}_{4}\right)$, filtered and concentrated in vacuo. Purification using flash column chromatography (Gradient: $1-10 \% \mathrm{MeOH} / \mathrm{CH}_{2} \mathrm{Cl}_{2}$ ) gave the crude mixture (130 mg, mixture of singly and doubly added products, $\mathbf{4 4}$ and $\mathbf{4 5}$ ) as a colourless oil which was used without further purification. The crude oil was dissolved in $\mathrm{MeOH}(18 \mathrm{~mL})$ before ammonium fluoride $(28 \mathrm{mg}, 0.77$ $\mathrm{mmol}$ ) was added and the reaction stirred at RT for $75 \mathrm{~min}$. The reaction mixture was concentrated in vacuo. Purification using flash column chromatography (Gradient: 10,15 then $20 \% \mathrm{MeOH} / \mathrm{CH}_{2} \mathrm{Cl}_{2}$ ) gave 46 (25 mg, 4\% over two steps) as a colourless oil; $\mathrm{R}_{\mathrm{f}} 0.23\left(15 \% \mathrm{MeOH} / \mathrm{CH}_{2} \mathrm{Cl}_{2}\right.$ ); $[\alpha]_{\mathrm{D}}{ }^{20}-0.3$ (c 1.2, $\mathrm{MeOH}$ ); IR $v_{\max }$ (oil) 3340 (NH), 3220 (NH), $3080(\mathrm{OH}), 2960$ - 2930 (CH), 1790 (C=O), 1730 (C=O), 1640 $(\mathrm{C}=0)$ and $1600(\mathrm{C}=\mathrm{C}) \mathrm{cm}^{-1}$; ${ }^{1} \mathrm{H}$ NMR $\left(600 \mathrm{MHz}, \mathrm{CD}_{3} \mathrm{OD}\right) \delta 8.29(1 \mathrm{H}, \mathrm{s}), 8.20(1 \mathrm{H}, \mathrm{s}), 6.02(1 \mathrm{H}, \mathrm{d}, J=4.5$ 
$\mathrm{Hz}), 4.74(1 \mathrm{H}, \mathrm{t}, J=5.0 \mathrm{~Hz}), 4.44-4.35(3 \mathrm{H}, \mathrm{m}), 4.28-4.24(1 \mathrm{H}, \mathrm{m}), 4.03(1 \mathrm{H}, \mathrm{d}, J=10.5 \mathrm{~Hz}), 3.88(1 \mathrm{H}$, $\mathrm{d}, J=10.5 \mathrm{~Hz}), 3.86(1 \mathrm{H}, \mathrm{s}), 3.50-3.44(4 \mathrm{H}, \mathrm{m}), 2.81-2.75(2 \mathrm{H}, \mathrm{m}), 2.66-2.63(4 \mathrm{H}, \mathrm{m}), 2.46-2.40$ $(2 \mathrm{H}, \mathrm{m}), 0.95(3 \mathrm{H}, \mathrm{s})$ and $0.92(3 \mathrm{H}, \mathrm{s}) ;{ }^{13} \mathrm{C} \mathrm{NMR}\left(150 \mathrm{MHz}, \mathrm{CD}_{3} \mathrm{OD}\right) \delta$ 175.5, 174.0, 173.9, 173.8, 157.6, $153.9,150.6,141.3,120.5,90.3,83.4,75.9,75.2,71.8,71.5,65.2,39.6,39.3,38.4,36.8,30.7,21.7$ and 20.8; HRMS calc'd for $\mathrm{C}_{50} \mathrm{H}_{73} \mathrm{~N}_{14} \mathrm{O}_{20} \mathrm{~S}_{2}$ expected 1253.4561, found 1253.4565 .

((2S,3R,4S,5S)-5-(6-Amino-9H-purin-9-yl)-3,4-dihydroxytetrahydrofuran-2-yl)methyl

$((R)-3-$ hydroxy-4-((3-((2-mercaptoethyl)amino)-3-oxopropyl)amino)-2,2-dimethyl-4-oxobutyl) succinate (10). To disulfide $46(15 \mathrm{mg}, 0.012 \mathrm{mmol})$ in $\mathrm{H}_{2} \mathrm{O}: \mathrm{MeCN}(60: 40,1 \mathrm{~mL})$ was added TCEP $(35 \mathrm{mg}$, $0.12 \mathrm{mmol}$ ). The reaction was stirred at RT for $1 \mathrm{~h}$. The reaction mixture was concentrated in vacuo. Purification using preparative HPLC (gradient 2, $\mathrm{r}_{\mathrm{t}} 10 \mathrm{~min}$ ) gave 10 (15 mg, $78 \%$ ) as a colourless gum; $\mathrm{R}_{\mathrm{f}} 0.48\left(15 \% \mathrm{MeOH} / \mathrm{CH}_{2} \mathrm{Cl}_{2} \text { ); [ } \alpha\right]_{\mathrm{D}}{ }^{20}-2.5$ (c 0.6, MeOH); IR $v_{\max }$ (oil) $3310(\mathrm{NH}), 3290(\mathrm{NH}), 3110(\mathrm{OH})$, $2940-2880(\mathrm{CH}), 1730(\mathrm{C}=\mathrm{O}), 1690(\mathrm{C}=\mathrm{O})$ and $1550(\mathrm{C}=\mathrm{C}) \mathrm{cm}^{-1} ;{ }^{1} \mathrm{H}$ NMR $\left(600 \mathrm{MHz}, \mathrm{CD}_{3} \mathrm{OD}\right) \delta 8.49(1 \mathrm{H}$, s), $8.40(1 \mathrm{H}, \mathrm{s}), 6.08(1 \mathrm{H}, \mathrm{d}, J=4.6 \mathrm{~Hz}), 4.74(1 \mathrm{H}, \mathrm{t}, J=4.9 \mathrm{~Hz}), 4.42-4.35(3 \mathrm{H}, \mathrm{m}), 4.30-4.27(1 \mathrm{H}, \mathrm{m})$, $4.01(1 \mathrm{H}, \mathrm{d}, J=10.5 \mathrm{~Hz}), 3.89(1 \mathrm{H}, \mathrm{d}, J=10.5 \mathrm{~Hz}), 3.85(1 \mathrm{H}, \mathrm{s}), 3.52-3.39(2 \mathrm{H}, \mathrm{m}), 3.33-3.28(2 \mathrm{H}, \mathrm{m})$, $2.66-2.63(2 \mathrm{H}, \mathrm{m}), 2.60-2.55(2 \mathrm{H}, \mathrm{m}), 2.45-2.41(2 \mathrm{H}, \mathrm{m}), 0.96(3 \mathrm{H}, \mathrm{s})$ and $0.93(3 \mathrm{H}, \mathrm{s}) ;{ }^{13} \mathrm{C} \mathrm{NMR}$ (150 MHz, CD 3 OD) $\delta 175.4,174.0,173.9,173.8,152.4,150.0,146.1,143.9,120.7,90.7,83.9,76.0$, 75.5, 71.7, 71.5, 65.9, 43.9, 39.3, 36.4, 29.9, 24.5, 21.6 and 20.8; HRMS calc'd for $\mathrm{C}_{25} \mathrm{H}_{38} \mathrm{~N}_{10} \mathrm{O}_{7} \mathrm{~S}$ expected 627.2395, found 627.2394 .

\subsection{Biological Assay}

Aurora A kinase activity was assayed by measuring incorporation of ${ }^{33} \mathrm{P}$ into myelin basic protein (Sigma). $100 \mathrm{ng}$ of purified recombinant Aurora A was incubated at room temperature for $30 \mathrm{~min}$ in a total volume of $15 \mu$ l containing $50 \mathrm{mM}$ HEPES $\mathrm{pH}$ 7.5, $10 \mathrm{mM} \mathrm{MgCl}, 1 \mathrm{mM}$ EGTA, 0.05\% Brij-35, $0.5 \mathrm{mg} / \mathrm{ml}$ myelin basic protein, and $5 \mu \mathrm{M} \gamma^{33} \mathrm{P}-\mathrm{ATP}(100 \mathrm{dpm} / \mathrm{pmol})$ in the presence or absence of $1 \mathrm{mM}$ DTT. The reaction was stopped by spotting the reaction mixture onto squares of P81 phosphocellulose ion exchange paper (Whatmann), which were then immersed in $1 \%(\mathrm{v} / \mathrm{v})$ phosphoric acid. After washing twice in $1 \%$ phosphoric acid followed by two washes in distilled water, the papers were air dried and radioactivity was counted by a scintillation counter. IC50 values were determined using GraphPad Prism version 6 (GraphPad Software, La Jolla California USA, www.graphpad.com).

\subsection{GOLD modelling}

For modelling the interaction between the enzyme and the inhibitors, the previously reported structure (PDB:1OL7 ${ }^{12}$ ) of active, phosphorylated human Aurora kinase $A$ was altered using $\mathrm{MOE}^{34}$ to 
include the cysteine residue Cys290, present in the activation loop of Aurora A kinase. GOLD (Genetic Optimisation for Ligand Docking $)^{32,33}$ was used for the in silico docking studies. All heteroatoms $\left(\mathrm{H}_{2} \mathrm{O}\right.$ and ligands) were removed from the PDB file and all polar hydrogens added. Ligand files were generated using MOE and used as .mol2 files. Each ligand structure was energy minimised using MOE prior to use in the GOLD docking. Since only interaction with the binding site was required, the existing ligand within the binding site was used (either AMP or ADP) to define the binding site and the default 10 genetic algorithm runs were run. The scoring function CHEMPLP ${ }^{67}$ was used to rank each of the resulting poses. The solutions were then exported as a .mol2 file and viewed with PyMOL.

\section{Declaration of Competing Interest}

The authors declare that they have no known competing financial interests or personal relationships that could have appeared to influence the work reported in this paper.

\section{Acknowledgments}

We are grateful to the EPSRC and the Department of Chemistry, UCL for a PhD studentship (EP/P505224/1 and EP/J500331/1, to FB), and to UCL for the award of an ORS PhD studentship (to TMT). We would like to thank UCL Business PLC for the award of a Proof of Concept Grant (UCLB 13014 and 11-018, to IG and ABT) and the BBSRC for the award of Responsive Mode grants on studying protein kinases and protein CoAlation (BB/L010410/1 and BB/S009027/1 to IG).

\section{References}

1. Pollard JR, Mortimore M. Discovery and Development of Aurora Kinase Inhibitors as Anticancer Agents. J Med Chem. 2009;52:2629-2651. doi:10.1021/jm8012129.

2. Keen N, Taylor S. Aurora-kinase inhibitors as anticancer agents. Nat Rev Cancer. 2004;4:927936. doi:10.1038/nrc1502.

3. Yan A, Wang L, Xu S, Xu J. Aurora-A kinase inhibitor scaffolds and binding modes. Drug Discovery Today. 2011;16:260-269. doi:10.1016/j.drudis.2010.12.003.

4. Carmena M, Earnshaw WC. The cellular geography of aurora kinases. Nat Rev Mol Cell Biol. 2003;4:842-854. doi:10.1038/nrm1245.

5. Bischoff JR, Anderson L, Zhu Y, Mossie K, Ng L, Souza B, Schryver B, Flanagan P, Clairvoyant F, Ginther C, C. Chan CSM, Novotny M, Slamon DJ, Plowman GD. A homologue of Drosophila aurora kinase is oncogenic and amplified in human colorectal cancers. EMBO J. 1998;17:30523065. doi:10.1093/emboj/17.11.3052.

6. Zhou H, Kuang J, Zhong L, Kuo W, Gray J, Sahin A, Brinkley B, Sen S. Tumour amplified kinase STK15/BTAK induces centrosome amplification, aneuploidy and transformation. Nat Genet. 1998;20:189-193. doi:10.1038/2496.

7. Goos JACM, Coupe VMH, Diosdado B, Diemen PMDV, Karga C, Belien JAM, Carvalho B, van den Tol MP, Verheul HMW, Geldof AA, Meijer GA, Hoekstra OS, Fijneman RJA. Aurora kinase A (AURKA) expression in colorectal cancer liver metastasis is associated with poor prognosis. Br J Cancer. 2013;109:2445-2452. doi:10.1038/bjc.2013.608.

8. Manfredi MG, Ecsedy JA, Meetze KA, Balani SK, Burenkova O, Chen W, Galvin KM, Hoar KM, Huck JJ, LeRoy PJ, Ray ET, Sells TB, Stringer B, Stroud SG, Vos TJ, Weatherhead GS, Wysong DR, 
Zhang MK, Bolen JB, Claiborne, CF. Antitumor activity of MLN8054, an orally active smallmolecule inhibitor of Aurora A kinase. Proc Natl Acad Sci U S A. 2007;104:4106-4111. doi:10.1073/pnas.0608798104.

9. Sloane DA, Trikic MZ, Chu MLH, Lamers MBAC, Mason CS, Mueller I, Savory WJ, Williams DH, Eyers PA. Drug-resistant aurora A mutants for cellular target validation of the small molecule kinase inhibitors MLN8054 and MLN8237. ACS Chem Biol. 2010;5:563-576. doi:10.1021/cb100053q.

10. Tayyar Y, Jubair L. Fallaha S, McMillan NAJ. Critical risk-benefit assessment of the novel anticancer aurora a kinase inhibitor alisertib (MLN8237): A comprehensive review of the clinical data. Crit Rev Oncol Hematol. 2017;119:59-65. doi:10.1016/j.critrevonc.2017.09.006.

11. D'Assoro AB, Haddad T, Galanis E. Aurora-A Kinase as a Promising Therapeutic Target in Cancer. Front Oncol. 2015;5:295. doi:10.3389/fonc.2015.00295.

12. Bayliss R, Sardon T, Vernos I, Conti E. Structural basis of Aurora-A activation by TPX2 at the mitotic spindle. Mol Cell. 2003;12:851-862. doi:10.1016/S1097-2765(03)00392-7.

13. Moore AS Blagg J, Linardopoulos S, Pearson ADJ. Aurora kinase inhibitors: novel small molecules with promising activity in acute myeloid and Philadelphia-positive leukemias. Leukemia. 2010;24:671-678. doi:10.1038/leu.2010.15.

14. McIntyre PJ, Collins PM, Vrzal L, Birchall K, Arnold LH, Mpamhanga C, Coombs PJ, Burgess SG, Richards MW, Winter A, Veverka V, von Delft F, Merritt A, Bayliss R. Characterization of Three Druggable Hot-Spots in the Aurora-A/TPX2 Interaction Using Biochemical, Biophysical, and Fragment-Based Approaches. ACS Chem Biol. 2017;12:2906-2914. doi:10.1021/acschembio.7b00537.

15. Pitsawong W, Buosi V, Otten R, Agafonov RV, Zorba A, Kern N, Kutter S, Kern G, Padua RAP, Meniche $\mathrm{X}$, Kern D. Dynamics of human protein kinase Aurora A linked to drug selectivity. Elife. 2018;7:e36656. doi:10.7554/eLife.36656.

16. Damodaran AP, Vaufrey L, Gavard O, Prigent C. Aurora A Kinase Is a Priority Pharmaceutical Target for the Treatment of Cancers. Trends Pharmacol Sci. 2017;38:687-700. doi:10.1016/j.tips.2017.05.003.

17. Zhang $\mathrm{H}$, Li Y, Wang $\mathrm{X}$, Wang Y. Probing the structural requirements of A-type Aurora kinase inhibitors using 3D-QSAR and molecular docking analysis. J Mol Model. 2012;18:1107-1122. doi:10.1007/s00894-011-1042-3.

18. Burgess SG, Mukherjee M, Sabir S, Joseph N, Gutierrez-Caballero C, Richards MW, HugueninDezot N, Chin JW, Kennedy EJ, Pfuhl M, Royle SJ, Gergely F, Bayliss, R. Mitotic spindle association of TACC3 requires Aurora-A-dependent stabilization of a cryptic alpha-helix. EMBO J. 2018;37:e97902. doi:10.15252/embj.201797902.

19. Theodoulou FL, Sibon OC, Jackowski S, Gout I. Coenzyme A and its derivatives: renaissance of a textbook classic. Biochem Soc Trans. 2014;42:1025-1032. doi: 10.1042/BST20140176

20. Leonardi R, Zhang YM, Rock CO, Jackowski S. Coenzyme A: back in action. Prog Lipid Res. 2005;44:125-153. 10.1016/j.plipres.2005.04.001

21. McAllister RA, Fixter, LM, Campbell EHG. The effect of tumour growth on liver pantothenate, CoA, and fatty acid synthetase activity in the mouse. Br J Cancer. 1988;57:83-86. 10.1038/bjc.1988.14

22. Reibel DK, Wyse BW, Berkich DA, Neely JR. Regulation of coenzyme A synthesis in heart muscle: effects of diabetes and fasting. Am J Physiol. 1981;240:H606-H611. https://doi.org/10.1152/ajpheart.1981.240.4.H606

23. Zhou B, Westaway SK, Levinson B, Johnson MA, Gitschier J, Hayflick SJ. A novel pantothenate kinase gene (PANK2) is defective in Hallervorden-Spatz syndrome. Nat Genet. 2001;28:345349. doi: $10.1038 / \mathrm{ng} 572$

24. Dusi, S., Valletta, L., Haack, T. B., Tsuchiya, Y., Venco, P., Pasqualato, S., Goffrini, P., Tigano, M., Demchenko, N., Wieland, T., et al. (2014). Exome sequence reveals mutations in CoA 
synthase as a cause of neurodegeneration with brain iron accumulation. Am. J. Hum. Genet. 94, 11-22.

25. Tsuchiya Y, Zhyvoloup A, Bakovic J, Thomas, N, Yu, BYK, Das, S, Orengo, C, Newell C, Ward J, Saladino G, Comitani F, Gervasio FL, Malanchuk OM, Khoruzhenko Al, Filonenko V, Peak-Chew SY, Skehel M, Gout I. Protein CoAlation and antioxidant function of coenzyme A in prokaryotic cells. Biochem J. 2018;475:1909-1937. doi: 10.1042/BCJ20180043

26. Tsuchiya Y, Peak-Chew SY, Newell C, Miller-Aidoo S, Mangal S, Zhyvoloup A, Bakovic J, Malanchuk O, Pereira GC, Kotiadis V, Szabadkai G, Duchen MR, Campbell M, Cuenca SR, VidalPuig A, James AM, Murphy MP, Filonenko V, Skehel M, Gout I. Protein CoAlation: a redoxregulated protein modification by coenzyme A in mammalian cells. Biochem J. 2017;474:24892508. doi: 10.1042/BCJ20170129

27. Gout I. Coenzyme A, protein CoAlation and redox regulation in mammalian cells. Biochem Soc Trans. 2018;46:721-728. doi: 10.1042/BST20170506

28. Aloum L, Brimson CA, Zhyvoloup A, Baines R, Bakovic J, Filonenko V, Thompson CRL, Gout I. Coenzyme $A$ and protein CoAlation levels are regulated in response to oxidative stress and during morphogenesis in Dictyostelium discoideum. Biochem Biophys Res Commun. 2019;511:294-299. 10.1016/j.bbrc.2019.02.031

29. Bakovic J, Yu BYK, Silva D, Chew SP, Kim S, Ahn SH, Palmer L, Aloum L, Stanzani G, Malanchuk $O$, Duchen MR, Singer M, Filonenko V, Lee TH, Skehel M, Gout, I. A key metabolic integrator, coenzyme $\mathrm{A}$, modulates the activity of peroxiredoxin 5 via covalent modification. Mol Cell Biochem. 2019;461:91-102. 10.1007/s11010-019-03593-w

30. Gout I. Coenzyme A: a protective thiol in bacterial antioxidant defence. Biochem Soc Trans. 2019;47:469-476. doi:10.1042/BST20180415

31. Tsuchiya Y, Byrne DP, Burgess SG, Bormann J, Baković J, Huang Y, Zhyvoloup A, Yu BYK, PeakChew S, Tran T, Bellany F, Tabor AB, Chan AWE, Guruprasad L, Garifulin O, Filonenko V, Vonderach M, Ferries S, Eyers CE, Carroll J, Skehel M, Bayliss R, Eyers PA, Gout I. Covalent Aurora A regulation by the metabolic integrator coenzyme A. Redox Biol. 2020;28:101318. doi: 10.1016/j.redox.2019.101318.

32. Jones $\mathrm{G}$, Willett $\mathrm{P}$, Glen RC. Molecular recognition of receptor sites using a genetic algorithm with a description of solvation. I Mol Biol. 1995;245:43-53. doi:10.1016/S00222836(95)80037-9.

33. Jones G, Willett $P$, Glen RC, Leach AR, Taylor R. Development and validation of a genetic algorithm for flexible docking. J Mol Biol. 1997;267:727-748. doi:10.1006/jmbi.1996.0897.

34. Molecular Operating Environment (MOE), 2019.0101; Chemical Computing Group Inc., 1010 Sherbrooke St. West, Suite \#910, Montreal, QC, Canada, H3A 2R7, 2015. http://www.chemcomp.com

35. Lipinski CA, Lombardo F, Dominy BW, Feeney PJ. Experimental and computational approaches to estimate solubility and permeability in drug discovery and development settings. Adv Drug Deliv Rev. 2001;46:3-26. doi:10.1016/S0169-409X(96)00423-1.

36. Lipinski CA. Drug-like properties and the causes of poor solubility and poor permeability. $J$ Pharmacol Toxicol Methods. 2000;44:235-249. doi:10.1016/S1056-8719(00)00107-6.

37. Elliott TS, Slowey A, Ye Y, Conway SJ. The use of phosphate bioisosteres in medicinal chemistry and chemical biology. MedChemComm. 2012;3:735-751. doi:10.1039/c2md20079a.

38. Meanwell NA. Synopsis of Some Recent Tactical Application of Bioisosteres in Drug Design. J Med Chem. 2011;54:2529-2591. doi:10.1021/jm1013693.

39. Rye CS, Baell JB. Phosphate isosteres in medicinal chemistry. Curr Med Chem. 2005;12:31273141. doi:10.2174/092986705774933452.

40. Erixon KM, Dabalos CL, Leeper FJ. Synthesis and biological evaluation of pyrophosphate mimics of thiamine pyrophosphate based on a triazole scaffold. Org. Biomol. Chem. 2008;6:3561-3572. doi:10.1039/b806580b. 
41. Erixon KM, Dabalos CL, Leeper FJ. Inhibition of pyruvate decarboxylase from Z-mobilis by novel analogues of thiamine pyrophosphate: investigating pyrophosphate mimics. Chem. Commun. 2007;7:960-962. doi:10.1039/b615861g.

42. Optibrium Ltd, Version 6.5.1-1-g2f87f001d (2018).

43. M. J. Waring. Lipophilicity in drug discovery. Exp Opin Drug Disc. 2010;5:235. doi: 10.1517/17460441003605098.

44. Morioka M, Kamizono A, Takikawa H, Mori A, Ueno H, Kadowaki S, Nakao Y, Kato K, Umezawa K. Design, synthesis, and biological evaluation of novel estradiol-bisphosphonateconjugates as bone-specific estrogens. Bioorg Med Chem. 2010;18:1143-1148. doi:10.1016/j.bmc.2009.12.041.

45. Tuck KL, Saldanha SA, Birch LM, Smith AG, Abell C. The design and synthesis of inhibitors of pantothenate synthetase. Org Biomol Chem. 2006;4:3598-3610. doi:10.1039/b609482a.

46. Manfredini S, Solaroli S, Angusti A, Nalin F, Durini E, Vertuani S, Pricl S, Ferrone M, Spadari S, Focher F, Verri A, De Clercq E, Balzarini J. Design and Synthesis of Phosphonoacetic Acid (PPA) Ester and Amide Bioisosters of Ribofuranosylnucleoside Diphosphates as Potential Ribonucleotide Reductase Inhibitors and Evaluation of Their Enzyme Inhibitory, Cytostatic and Antiviral Activity. Antiviral Chem Chemother. 2003;14:183-194. doi:10.1177/095632020301400403.

47. Sierzchala AB, Dellinger DJ, Betley JR, Wyrzykiewicz TK, Yamada CM, Caruthers MH. Solidphase oligodeoxynucleotide synthesis: A two-step cycle using peroxy anion deprotection. $J$ Am Chem Soc. 2003;125:13427-13441. doi:10.1021/ja030376n.

48. Virga KG, Zhang YM, Leonardi R, Ivey RA, Hevener K, Park HW, Jackowski S, Rock CO, R. Lee RE. Structure-activity relationships and enzyme inhibition of pantothenamide-type pantothenate kinase inhibitors. Bioorg Med Chem. 2006;14:1007-1020. doi:10.1016/j.bmc.2005.09.021.

49. Yan H, Oh J-S, Song CE. A mild and efficient method for the selective deprotection of silyl ethers using KF in the presence of tetraethylene glycol. Org Biomol Chem. 2011;9:8119-8121. doi:10.1039/c1ob06300f.

50. Ranu BC, Jana $U$, Majee A. A simple and efficient method for selective deprotection of $t$ butyldimethylsilyl ethers by zinc tetrafluoroborate in water. Tetrahedron Lett. 1999;40:19851988. doi:10.1016/S0040-4039(99)00097-0.

51. O'Mahony G, Svensson S, Sundgren A, Grotli M. Synthesis of 2'-([1,2,3]triazol-1-yl)-2'deoxyadenosines. Nucleosides Nucleotides Nucleic Acids. 2008;27:449-459. doi:10.1080/15257770802086880.

52. Yao Y, Chen P, Diao J, Cheng G, Deng L, Anglin JL, Prasad BVV, Song Y. Selective Inhibitors of Histone Methyltransferase DOT1L: Design, Synthesis, and Crystallographic Studies. J Am Chem Soc. 2011;133:16746-16749. doi:10.1021/ja206312b.

53. Kim E-K, Switzer C. Bis(6-carboxypurine)-Cu' ${ }^{2+}$ A Possibly Primitive Metal-Mediated Nucleobase Pair. Org Lett. 2014:16;4059-4061. doi:10.1021/ol5018728.

54. Kulikova IV, Muradova DA, Mikhailov SN. Effective isomerization of 3 ',5 '-O(tetraisopropyldisiloxane-1,3-diyl)nucleosides in the presence of trimethylsilyl trifluoromethanesulfonate. Arkivoc. 2009;158-170. doi:10.3998/ark.5550190.0010.313.

55. Petersen SG, Rajski SR. o-Nitrobenzenesulfonamides in nucleoside synthesis: Efficient 5'aziridination of adenosine. J Org Chem. 2005;70:5833-5839. doi:10.1021/jo050205w.

56. Matulic-Adamic J, Gonzalez C, Usman N, Beigelman L. Synthesis of pyridinone ribonucleoside 3'-O-phosphoramidites and their incorporation into oligoribonucleotides. Bioorg Med Chem Lett. 1996;6:373-378. doi:10.1016/0960-894X(96)00030-3.

57. Smith M, Rammler DH, Goldberg IH, Khorana HG. Studies on Polynucleotides. XIV. Specific Synthesis of the C3"-C5" Interribonucleotide Linkage. Syntheses of Uridylyl-(3" $\rightarrow 5$ ")-Uridine and Uridylyl-(3" $\rightarrow 5$ ")-Adenosine. J Am Chem Soc. 1962;84:430-440. doi:10.1021/ja00862a023. 
58. Peng L, DeSousa J, Su Z, Novak BM, Nevzorov AA, Garland ER, Melander C. Inhibition of Acinetobacter baumannii biofilm formation on a methacrylate polymer containing a 2aminoimidazole subunit. Chem Commun. 2011;47:4896-4898. doi:10.1039/c1cc10691k.

59. Moolman WJA, de Villiers M, Strauss E. Recent advances in targeting coenzyme A biosynthesis and utilisation for antimicrobial drug development. Biochem Soc Trans. 2014;42:1080-1086. doi:10.1042/BST20140131.

60. Saliba KJ, Spry C. Exploiting the coenzyme A biosynthesis pathway for the identification of new antimalarial agents: the case for pantothenamides. Biochem Soc Trans. 2014;42:1087-1093. doi:10.1042/BST20140158.

61. Guan JM, Barnard L, Cresson J, Hoegl A, Chang JH, Strauss E, Auclair K. Probing the ligand preferences of the three types of bacterial pantothenate kinase. Bioorg Med Chem. 2018;26:5896-5902. doi:10.1016/j.bmc.2018.10.042.

62. Jansen PAM, van Der Krieken DA, Botman PNM, Blaauw RH, Cavina L, Raaijmakers EM, de Heuvel E, Sandrock J, Pennings L, Hermkens PHH, Zeeuwen PLIM, Rutjes FPJT, Schalkwijk, J. Stable pantothenamide bioisosteres: novel antibiotics for Gram-positive bacteria. J Antibiot. 2019;72:682-692. doi: 10.1038/s41429-019-0196-6.

63. Sledz P, Silvestre HL, Hung AW, Ciulli A, Blundell TL, Abell C. Optimization of the Interligand Overhauser Effect for Fragment Linking: Application to Inhibitor Discovery against Mycobacterium tuberculosis Pantothenate Synthetase. J Am Chem Soc. 2010;132:4544-4545. doi:10.1021/ja100595u.

64. Liu Q, Cai X, Yang D, Chen Y, Wang Y, Shao L, Wang M. Cycloalkane analogues of sinefungin as EHMT1/2 inhibitors. Bioorg Med Chem. 2017; 25:4579-4594. doi:10.1016/j.bmc.2017.06.032.

65. Beaton G, Jones AS, Walker RT. The chemistry of 2',3'-seconucleosides. 3. Synthesis and reactions of purine-2', $3^{\prime}$-secoribonucleosides. Tetrahedron. 1988;44:6419-6428. doi: 10.1016/S0040-4020(01)89829-X.

66. Isomura $S$, Wirsching $P$, Janda KD. An immunotherapeutic program for the treatment of nicotine addition: Hapten design and synthesis. J Org Chem. 2001;66:4115-4121. doi: 10.1021/jo001442w.

67. Korb O, Stützle T, Exner TE. Empirical Scoring Functions for Advanced Protein-Ligand Docking with PLANTS. J Chem Inf Model. 2009;49:84-96. doi: 10.1021/ci800298z. 